\title{
GAUSS-GREEN THEOREM FOR WEAKLY DIFFERENTIABLE VECTOR FIELDS, SETS OF FINITE PERIMETER, AND BALANCE LAWS
}

\author{
GUI-QIANG CHEN MONICA TORRES WILLIAM P. ZIEMER
}

\begin{abstract}
We analyze a class of weakly differentiable vector fields $\boldsymbol{F}: \mathbb{R}^{N} \rightarrow \mathbb{R}^{N}$ with the property that $\boldsymbol{F} \in L^{\infty}$ and $\operatorname{div} \boldsymbol{F}$ is a Radon measure. These fields are called bounded divergencemeasure fields. The primary focus of our investigation is to introduce a suitable notion of the normal trace of any divergence-measure field $\boldsymbol{F}$ over the boundary of an arbitrary set of finite perimeter, which ensures the validity of the Gauss-Green theorem. To achieve this, we first develop an alternative way to establish the Gauss-Green theorem for any smooth bounded set with $\boldsymbol{F} \in L^{\infty}$. Then we establish a fundamental approximation theorem which states that, given a Radon measure $\mu$ that is absolutely continuous with respect to $\mathcal{H}^{N-1}$ on $\mathbb{R}^{N}$, any set of finite perimeter can be approximated by a family of sets with smooth boundary essentially from the measure-theoretic interior of the set with respect to the measure $\|\mu\|$. We employ this approximation theorem to derive the normal trace of $\boldsymbol{F}$ on the boundary of any set of finite perimeter, $E$, as the limit of the normal traces of $\boldsymbol{F}$ on the boundaries of the approximate sets with smooth boundary, so that the Gauss-Green theorem for $\boldsymbol{F}$ holds on $E$. With these results, we analyze the Cauchy fluxes that are bounded by a Radon measure over any oriented surface (i.e. an $(N-1)$ dimensional surface that is a part of the boundary of a set of finite perimeter) and thereby develop a general mathematical formulation of the physical principle of balance law through the Cauchy flux. Finally, we apply this framework to the derivation of systems of balance laws with measurevalued source terms from the formulation of balance law. This framework also allows the recovery of Cauchy entropy fluxes through the Lax entropy inequality for entropy solutions of hyperbolic conservation laws.
\end{abstract}

\section{INTRODUCTION}

In this paper we analyze a class of weakly differentiable vector fields $\boldsymbol{F}: \mathbb{R}^{N} \rightarrow \mathbb{R}^{N}$ with the property that $\boldsymbol{F} \in L^{\infty}$ and $\operatorname{div} \boldsymbol{F}$ is a Radon measure $\mu$ with finite total variation (i.e. a totally finite signed measure). These fields are called bounded divergence-measure fields, and the class is denoted by $\mathcal{D} \mathcal{M}^{\infty}$. The primary focus of our investigation is to introduce a suitable notion of the normal trace of any divergence-measure field over the boundary of an arbitrary set of finite perimeter to obtain a general version of the Gauss-Green theorem. Clearly, this investigation is closely related to the theory of $B V$ functions in $\mathbb{R}^{N}$; in fact, it would be completely subsumed by the $B V$ theory if the fields were of the form $\boldsymbol{F}=\left(F_{1}, F_{2}, \ldots, F_{N}\right)$ with each $F_{k} \in B V\left(\mathbb{R}^{N}\right)$, since then $\operatorname{div} \boldsymbol{F}=\sum_{k=1}^{N} \frac{\partial F_{k}}{\partial x_{k}}$ is a Radon measure $\mu$ with finite total variation (cf. [Zie89]). However, in general, the condition div $\boldsymbol{F}=\mu$ allows for cancellation, which thus makes the problem more difficult and accordingly more important for applications (see 99-11). For the Gauss-Green theorem in the $B V$ setting, we refer to Burago-Maz'ja [BM69, Volpert [Vol67a], and the references therein. The Gauss-Green theorem for Lipschitz vector fields over sets of finite perimeter was first obtained by DeGiorgi DG61a, DG61b and Federer Fed45, Fed58. Also see Evans-Gariepy EG92, Lin-Wang LY02, and Simon Sim83.

1991 Mathematics Subject Classification. Primary: 28C05, 26B20, 28A05, 26B12, 35L65, 35L50; Secondary: 28A75, 28A25, 26B05, 26B30, 26B40.

Key words and phrases. Gauss-Green theorem, weakly differential vector fields, divergence-measure fields, sets of finite perimeter, normal traces, balance law, oriented surfaces, Cauchy fluxes, axioms for continuum thermodynamics, field equations, entropy solutions, conservation laws.

Submitted on September 3, 2007. 
Some earlier efforts were made on generalizing the Gauss-Green theorem for some special situations of divergence-measure fields, and relevant results can be found in Anzellotti Anz83 for an abstract formulation when $\boldsymbol{F} \in L^{\infty}$ over a set with $C^{1}$ boundary and Ziemer [Zie83] for a related problem for $\operatorname{div} \boldsymbol{F} \in L^{\infty}$; also see [AFP00, BC84, BF91, JN90, JN94b, JNm95, Non95, Pfe05a, DPP04a, Pfe01, LRX07. In Chen-Frid CF99a, CF03, an explicit way to formulate the suitable normal traces over Lipschitz deformable surfaces was first observed for $\boldsymbol{F} \in \mathcal{D} \mathcal{M}^{\infty}$. In particular, it was proved in CF99a, CF03] that the normal trace over a Lipschitz deformable surface, oriented by the unit normal vector $\nu$, is determined completely by the neighborhood information from the positive side of the surface pointed by $\nu$ and is independent of the information from the other side. This is the primary motivation for our further investigation on divergence-measure fields. Chen-Torres CT05 were the first to obtain the normal trace for any bounded divergence-measure field over a set of finite perimeter, $E$, and the corresponding Gauss-Green theorem. One of the main results in this paper is to obtain this normal trace as the limit of the normal traces over the smooth boundaries that approximate the reduced boundary $\partial^{*} E$ of $E$. In particular, the normal trace is determined completely by the neighborhood information essentially from the measure-theoretic interior of the set (see Theorem 21.1), so that the Gauss-Green theorem holds for any set of finite perimeter.

We recall a very general approach, initiated by Fuglede Fug60, in which the following result was established: If $\boldsymbol{F} \in L^{p}\left(\mathbb{R}^{N} ; \mathbb{R}^{N}\right), 1 \leq p \leq \infty$, is a vector field with $\operatorname{div} \boldsymbol{F}=\mu$, where $\mu$ is a signed Radon measure, then

$$
\int_{E} \operatorname{div} \boldsymbol{F}:=\mu(E)=-\int_{\partial^{*} E} \boldsymbol{F}(y) \cdot \nu(y) d \mathcal{H}^{N-1}(y)
$$

for "almost all" sets of finite perimeter, $E$, where $\mathcal{H}^{N-1}$ is the $(N-1)$-dimensional Hausdorff measure. The term "almost all" is expressed in terms of a condition that resembles "extremal length", a concept used in complex analysis and potential theory (cf. Zie67, Zie69, Zie70, He07). One way of summarizing our work in this paper is to say that we wish to extend Fuglede's result so that (2.1) holds for every set $E$ of finite perimeter. Of course, this requires a suitable notion of the normal trace of $\boldsymbol{F}$ on $\partial^{*} E$. This is really the crux of the problem as $\boldsymbol{F}$, being only measurable, cannot be re-defined on an arbitrary set of dimension $N-1$. To achieve our goal, we first establish a fundamental approximation theorem which states that, given a Radon measure $\mu$ on $\mathbb{R}^{N}$ such that $\mu<<\mathcal{H}^{N-1}$, any set of finite perimeter can be approximated by a family of sets with smooth boundary essentially from the measure-theoretic interior of the set with respect to the measure $\|\mu\|$ (e.g. $\mu=\operatorname{div} \boldsymbol{F}$ ). Then we employ this approximation theorem to derive the normal trace of $\boldsymbol{F}$ on the boundary of any set of finite perimeter as the limit of the normal traces of $\boldsymbol{F}$ on the smooth boundaries of the approximate sets and establish the Gauss-Green theorem for $\boldsymbol{F}$ which holds for an arbitrary set of finite perimeter.

With these results on divergence-measure fields and sets of finite perimeter, we analyze the Cauchy flux that is bounded by a nonnegative Radon measure $\sigma$ over an oriented surface (i.e. an $(N-1)$ dimensional surface that is a part of the boundary of a set of finite perimeter) and develop a general mathematical formulation of the physical principle of balance law through the Cauchy flux. In the classical setting of the physical principle of balance law, Cauchy Ca1823, Ca1827] first discovered that the flux density is necessarily a linear function of the interior normal (equivalently, the exterior normal) under the assumption that the flux density through a surface depends on the surface solely through the normal at that point. It was shown in Noll [Nol59 that Cauchy's assumption follows from the balance law. Ziemer Zie83 provided a first formulation of the balance law for the flux function $\boldsymbol{F} \in L^{\infty}$ with $\operatorname{div} \boldsymbol{F} \in L^{\infty}$ at the level of generality with sets of finite perimeter. Also see Dafermos [Daf98] and Gurtin-Martins [GW67, GW71]. One of the new features in our formulation is to allow the presence of exceptional surfaces, "shock waves", across which the Cauchy flux has a jump. When the Radon measure $\sigma$ reduces to the $N$-dimensional Lebesgue measure $\mathcal{L}^{N}$, the formulation reduces to Ziemer's formulation in [Zie83, which shows its consistency with the classical setting. We first show that, for a Cauchy flux $\mathcal{F}$ bounded by a measure $\sigma$, there exists a bounded 
divergence-measure field $\boldsymbol{F}: \mathbb{R}^{N} \rightarrow \mathbb{R}^{N}$, defined $\mathcal{L}^{N}$-a.e., such that

$$
\mathcal{F}(S)=-\int_{S} \boldsymbol{F}(y) \cdot \nu(y) d \mathcal{H}^{N-1}(y)
$$

for almost any oriented surface $S$, oriented by the interior unit normal $\nu$. Then we employ our results on divergence-measure fields to recover the values of the Cauchy flux on the exceptional surfaces directly via the vector field $\boldsymbol{F}$. The value as the normal trace of $\boldsymbol{F}$ on the exceptional surface is the unique limit of the normal traces of $\boldsymbol{F}$ on the non-exceptional surfaces essentially from the positive side of the exceptional surface pointed by $\nu$. Finally, we apply this general framework to the derivation of systems of balance laws with measure-valued source terms from the mathematical formulation of balance law. We also apply the framework to the recovery of Cauchy entropy fluxes through the Lax entropy inequality for entropy solutions of hyperbolic conservation laws by capturing entropy dissipation.

We observe the recent important work by Bourgain-Brezis BB02] and De Pauw-Pfeffer [DPP04a] for the following problem with different view of point: Find a continuous vector field to the divergence-measure equation:

$$
\operatorname{div} \boldsymbol{F}=\mu \quad \text { in } \Omega,
$$

for a given Radon measure $\mu$. In the case $d \mu=f d x$ where $f \in L_{l o c}^{n}(\Omega)$, the existence of a solution $\boldsymbol{F}$ to (3.1) follows form the closed-range or Hahn-Banach theorem as shown in BB02]. It is proved in DPP04a] that equation (3.1) has a continuous weak solution if and only if $\mu$ is a strong charge, i.e., given $\varepsilon>0$ and a compact set $K \subset \Omega$, there is $\theta>0$ such that

$$
\int_{\Omega} \phi d \mu \leq \varepsilon\|\nabla \phi\|_{L^{1}}+\theta\|\phi\|_{L^{1}}
$$

for any smooth function $\phi$ compactly supported on $K$.

The organization of this paper is as follows. In $\S 2$, we first recall some properties of Radon measures, sets of finite perimeter, and related $B V$ functions, and then we introduce the notion of an oriented surface and develop some basic properties of divergence-measure fields. In $\S 3$, we develop an alternative way to obtain the Gauss-Green formula for a bounded divergence-measure field over any smooth boundary by a technique, which motivates our further development for the general case. In $\S 4$, we establish a fundamental approximation theorem which states that, given a Radon measure $\mu$ on $\mathbb{R}^{N}$ such that $\mu<<\mathcal{H}^{N-1}$, any set of finite perimeter can be approximated by a sequence of sets with smooth boundary essentially from the interior of the set with respect to the measure $\|\mu\|$. In $\S 5$, we introduce the normal trace of a divergence-measure field $\boldsymbol{F}$ on the boundary $\partial E$ of any set of finite perimeter as the limit of the normal traces of $\boldsymbol{F}$ on the smooth surfaces that approximate $\partial E$ essentially from the measure-theoretic interior of $E$ with respect to the measure $\|\operatorname{div} \boldsymbol{F}\|$, constructed in $\S 4$, and then we establish the corresponding Gauss-Green theorem. In $\S 6-\S 7$, we further analyze properties of divergence-measure fields, especially showing the representation of the divergence-measures of jump sets via the normal traces and the consistency of our normal traces with the classical traces (i.e. values) when the vector field is continuous. In $\S 8$, we first show that, if the set of finite perimeter, $E$, satisfies (29.1) (which is similar to Lewis's "uniformly fat" condition in potential theory Lew88a] , there exists a one-sided approximation to $E$, and we then show that an open set of finite perimeter is an extension domain for any bounded divergence-measure field. In $\S 9$, we first introduce a class of Cauchy fluxes that allow the presence of these exceptional surfaces or "shock waves", and we then prove that such a Cauchy flux induces a bounded divergence-measure (vector) field $\boldsymbol{F}$ so that the Cauchy flux over every oriented surface with finite perimeter can be recovered through $\boldsymbol{F}$ via the normal trace over the oriented surface.

In $\S 10$, we apply the results established in $\S 3-\S 9$ to the mathematical formulation of the physical principle of balance law and the rigorous derivation of systems of balance laws with measure-valued source terms from that formulation. Finally, in $\S 11$, we apply our results to the recovery of Cauchy 
entropy flux through the Lax entropy inequality for entropy solutions of hyperbolic conservation laws by capturing entropy dissipation.

\section{Radon Measures, Sets of finite perimeter, and Divergence-Measure Fields}

In this section we first recall some properties of Radon measures, sets of finite perimeter, and related $B V$ functions (also cf. [AFP00, EG92, Fed69, Giu77, Zie89]). We then introduce the notion of oriented surfaces and develop some basic properties of divergence-measure fields. For the sake of completeness, we start with some basic notions and definitions. First, denote by $\mathcal{H}^{M}$ the $M$ dimensional Hausdorff measure in $\mathbb{R}^{N}$ for $M \leq N$, and by $\mathcal{L}^{N}$ the Lebesgue measure in $\mathbb{R}^{N}$ (recall that $\mathcal{L}^{N}=\mathcal{H}^{N}$ ). For any $\mathcal{L}^{N}$-measurable set $E \subset \mathbb{R}^{N}$, we denote $|E|$ as the $\mathcal{L}^{N}$-Lebesgue measure of the set $E$ and $\partial E$ as its topological boundary. Also, we denote $B(x, r)$ as the closed ball of radius $r$ and center at $x$. The symmetric difference of sets is denoted by

$$
A \Delta B:=(A \backslash B) \cup(B \backslash A) .
$$

Let $\Omega \subset \mathbb{R}^{N}$ be open. We denote by $E \Subset \Omega$ that the closure of $E$ is compact and contained in $\Omega$. Let $C_{c}(\Omega)$ be the space of compactly supported continuous functions on $\Omega$ with $\|\varphi\|_{0 ; \Omega}:=\sup \{|\varphi(y)|:$ $y \in \Omega$ \}. A Radon measure $\mu$ in $\Omega$ is a regular Borel measure whose total variation on each compact set $K \Subset \Omega$ is finite, i.e. $\|\mu\|(K)<\infty$. The space of Radon measures supported on an open set $\Omega$ is denoted by $\mathcal{M}(\Omega)$. Any Radon measure $\mu$ can be decomposed into the difference of two positive Radon measures $\mu=\mu^{+}-\mu^{-}$; the total variation of $\mu$ is $\|\mu\|=\mu^{+}+\mu^{-}$. Equivalently, if $\mu$ is a signed Radon measure on $\Omega$, the total variation of $\mu$ on any bounded open set $B \subset \Omega$ is equal to

$$
\|\mu\|(B)=\sup \left\{\int_{\Omega} \varphi d \mu: \varphi \in C_{c}(B),\|\varphi\|_{0 ; \Omega} \leq 1\right\}=\sup \left\{\sum_{i=0}^{\infty}\left|\mu\left(B_{i}\right)\right|\right\}
$$

where the second supremum is taken over all pairwise disjoint Borel sets $B_{i}$ with $B=\bigcup_{i=1}^{\infty} B_{i}$. Since the space of Radon measures can be identified with the dual of $C_{c}(\Omega)$, we may consider a Radon measure $\mu$ as a linear functional on $C_{c}(\Omega)$, written as

$$
\mu(\varphi):=\int_{\Omega} \varphi d \mu \quad \text { for each } \varphi \in C_{c}(\Omega) .
$$

We recall the familiar weak*-topology on $\mathcal{M}(\Omega)$ which, when restricted to a sequence $\left\{\mu_{k}\right\}$, yields

$$
\mu_{k} \stackrel{*}{\rightarrow} \mu \quad \text { in } \mathcal{M}(\Omega),
$$

that is, $\mu_{k}$ converges to $\mu$ in the weak* topology if and only if

$$
\mu_{k}(\varphi) \rightarrow \mu(\varphi) \quad \text { for each } \varphi \in C_{c}(\Omega) .
$$

The space $L^{p}(\Omega, \mu), 1 \leq p \leq \infty$, denotes all the functions $f$ with the property that $|f|^{p}$ is $\mu$ integrable. The conjugate of $p$ is $q:=p /(p-1)$. The $L^{p}$ norm of $f$ on a set $E$ with integration taken with respect to a measure $\mu$ is denoted by $\|f\|_{p ; E, \mu}$. In the event $\mu$ is Lebesgue measure, we will simply write $\|f\|_{p ; E}$.

4.1. Theorem (Uniform boundedness principle). Let $X$ be a Banach space. If $T_{k}$ is a sequence of linear functionals on $X$ which converge weak* to $T$. Then

$$
\limsup _{k \rightarrow \infty}\left\|T_{k}\right\|<\infty
$$

This theorem implies the following corresponding result for Radon measures.

4.2. Corollary. Let $\mu_{k}$ be a sequence of Radon measures that converge to $\mu$ in the weak* topology. Then

$$
\limsup _{k \rightarrow \infty}\left\|\mu_{k}\right\|<\infty
$$


Next, we quote a familiar result concerning weak*-convergence.

4.3. Lemma. Let $\mu$ be a Radon measure on $\Omega$ and let $\mu_{k}$ be a sequence of Radon measures converging weak ${ }^{*}$ to $\mu$. Then

(i) If $A \subset \Omega$ is any open set and $\mu_{k}$ are positive Radon measures,

$$
\mu(A) \leq \liminf _{k \rightarrow \infty} \mu_{k}(A)
$$

(ii) If $K \subset \Omega$ is any compact set and $\mu_{k}$ are positive Radon measures,

$$
\mu(K) \geq \limsup _{k \rightarrow \infty} \mu_{k}(K)
$$

(iii) If $\left\|\mu_{k}\right\| \stackrel{*}{\rightarrow} \sigma$, then $\|\mu\| \leq \sigma$. In addition, if $E$ satisfies $\sigma(\partial E)=0$, then

$$
\mu(E)=\lim _{k \rightarrow \infty} \mu_{k}(E) .
$$

More generally, if $f$ is a bounded Borel function with compact support in $\Omega$ such that the set of its discontinuity points is $\sigma$-negligible, then

$$
\lim _{k \rightarrow \infty} \int_{\Omega} f d \mu_{k}=\int_{\Omega} f d \mu .
$$

5.1. Definition. For every $\alpha \in[0,1]$ and every $\mathcal{L}^{N}$-measurable set $E \subset \mathbb{R}^{N}$, define

$$
E^{\alpha}:=\left\{y \in \mathbb{R}^{N}: D(E, y)=\alpha\right\}
$$

where

$$
D(E, y):=\lim _{r \rightarrow 0} \frac{|E \cap B(y, r)|}{|B(y, r)|} .
$$

Then $E^{\alpha}$ is the set of all points with density $\alpha$. We define the measure-theoretic boundary of $E$, $\partial^{m} E$, as

$$
\partial^{m} E:=\mathbb{R}^{N} \backslash\left(E^{0} \cup E^{1}\right) .
$$

5.2. Definition. A function $f: \Omega \rightarrow \mathbb{R}$ is called a function of bounded variation if each partial derivative of $f$ is a totally finite signed Radon measure. Notationally, we write $f \in B V(\Omega)$. Let $E \Subset \Omega$ be an $\mathcal{L}^{N}$-measurable subset. We say that $E$ is a set of finite perimeter if $\mathcal{H}^{N-1}\left(\partial^{m} E\right)<\infty$. Equivalently, $E$ is of finite perimeter if $\chi_{E} \in B V(\Omega)$. Consequently, if $E$ is a set of finite perimeter, then $\nabla \chi_{E}$ is a (vector-valued) Radon measure whose total variation is denoted by $\left\|\nabla \chi_{E}\right\|$.

5.3. Definition. Let $E \Subset \Omega$ be a set of finite perimeter. The reduced boundary of $E$, denoted as $\partial^{*} E$, is the set of all points $y \in \Omega$ such that

(i) $\left\|\nabla \chi_{E}\right\|(B(y, r))>0$ for all $r>0$;

(ii) The limit $\nu_{E}(y):=\lim _{r \rightarrow 0} \frac{\nabla \chi_{E}(B(y, r))}{\left\|\nabla \chi_{E}\right\|(B(y, r))}$ exists.

Then, for $\mathcal{H}^{N-1}$-a.e. $y \in \partial^{*} E$,

$$
\lim _{r \rightarrow 0} \frac{\left\|\nabla \chi_{E}\right\|(B(y, r))}{\alpha(N-1) r^{N-1}}=1,
$$

where $\alpha(N-1)$ is the Lebesgue measure of the unit ball in $\mathbb{R}^{N-1}$, and the generalized gradient of $\chi_{E}$ enjoys the following basic relationship with $\mathcal{H}^{N-1}$ :

$$
\left\|\nabla \chi_{E}\right\|=\mathcal{H}^{N-1}\left\llcorner\partial^{*} E .\right.
$$

The unit vector, $\boldsymbol{\nu}_{E}(y)$, is called the measure-theoretic interior unit normal to $E$ at $y$ (we sometimes write $\boldsymbol{\nu}$ instead of $\boldsymbol{\nu}_{E}$ for notational simplicity). Also, we recall that the reduced boundary, $\partial^{*} E$, is an $(N-1)$-rectifiable set which implies that there exists a countable family of $C^{1}$-manifolds $M_{k}$ of dimension $N-1$ and a set $\mathcal{N}$ of $\mathcal{H}^{N-1}$ measure zero such that

$$
\partial^{*} E \subset\left(\bigcup_{k=1}^{\infty} M_{k}\right) \cup \mathcal{N} .
$$


In view of the following, we see that $\boldsymbol{\nu}=\boldsymbol{\nu}_{E}$ is aptly named because $\boldsymbol{\nu}$ is the interior unit normal to $E$ provided that $E$ (in the limit and in measure) lies in the appropriate half-space determined by the hyperplane orthogonal to $\boldsymbol{\nu}$; that is, $\boldsymbol{\nu}$ is the interior unit normal to $E$ at $x$ provided that

$$
D(\{y:(y-x) \cdot \boldsymbol{\nu}>0, y \notin E\} \cup\{y:(y-x) \cdot \boldsymbol{\nu}<0, y \in E\}, y)=0 .
$$

We will refer to the sets $E^{0}$ and $E^{1}$ as the measure-theoretic exterior and interior of $E$. We note that, in general, the sets $E^{0}$ and $E^{1}$ do not coincide with the topological exterior and interior of the set $E$. The sets $E^{0}$ and $E^{1}$ also motivate the definition of measure-theoretic boundary. Indeed, for any set $E \subset \mathbb{R}^{N}$, the definitions imply that $\mathbb{R}^{N}=E^{1} \cup \partial^{*} E \cup E^{0} \cup \mathcal{N}$ where $\mathcal{H}^{N-1}(\mathcal{N})=0$. If we define a set $E$ to be "open" if $E$ is both measurable and $D(E, x)=1$ for all $x \in E$, then this concept of openness defines a topology, called the density topology. It is an interesting exercise to prove that the open sets are closed under arbitrary unions; the crux of the problem is to prove that the arbitrary union is, in fact, measurable. This topology is significant because it is the smallest topology (the one with the smallest number of open sets) for which the approximately continuous functions are continuous GNN61.

6.1. Remark. If $E$ is a set of finite perimeter, then clearly

$$
\partial^{*} E \subset E^{\frac{1}{2}} \subset \partial^{m} E, \quad \mathcal{H}^{N-1}\left(\partial^{m} E \backslash \partial^{*} E\right)=0 .
$$

The following result, which is easily verified (although tedious), will be needed in the sequel.

6.2. Lemma. If $A, B \Subset \Omega$ are sets of finite perimeter, then

$$
\partial^{m}(A \cap B)=\left(\partial^{m} A \cap B\right) \cup\left(A \cap \partial^{m} B\right) \cup\left(\partial^{m} A \cap \partial^{m} B\right) .
$$

6.3. Definition. Let $\rho \in C_{c}^{\infty}\left(\mathbb{R}^{N}\right)$ be a standard symmetric mollifying kernel; that is, $\rho$ is a nonnegative function with support in the unit ball and satisfies $\|\rho\|_{1 ; \mathbb{R}^{N}}=1$. With $u \in L^{1}\left(\mathbb{R}^{N}\right)$, we set $u_{\varepsilon}:=u * \rho_{\varepsilon}$, where the sequence $\rho_{\varepsilon}(y):=\frac{1}{\varepsilon^{N}} \rho\left(\frac{y}{\varepsilon}\right)$ forms a mollifier.

Recall the following properties of mollification (cf. [Zie89]):

6.4. Lemma. (i) If $u \in L_{l o c}^{1}\left(\mathbb{R}^{N}\right)$, then, for every $\varepsilon>0, u_{\varepsilon} \in C^{\infty}\left(\mathbb{R}^{N}\right)$ and $D^{\alpha}\left(\rho_{\varepsilon} * u\right)=$ $\left(D^{\alpha} \rho_{\varepsilon}\right) * u$ for each multi-index $\alpha$;

(ii) With $k:=1 / \varepsilon_{k}$ and $\varepsilon_{k} \rightarrow 0, u_{k}(x) \rightarrow u(x)$ whenever $x$ is a Lebesgue point of $u$. In particular, if $u$ is continuous, then $u_{\varepsilon}$ converges uniformly to $u$ on compact subsets of $\mathbb{R}^{N}$.

When $u$ is taken as $\chi_{E}$ for a set of finite perimeter, $E$, this result can be considerably strengthened.

6.5. Lemma. If $u_{k}$ is the mollification of $\chi_{E}$ for a set of finite perimeter, $E$, then the following hold:

(i) $u_{k} \in C^{\infty}\left(\mathbb{R}^{N}\right)$;

(ii) There is a set $\mathcal{N}$ with $\mathcal{H}^{N-1}(\mathcal{N})=0$ and a function $u_{E} \in B V$ such that, for all $y \notin \mathcal{N}$, $u_{k}(y) \rightarrow u_{E}(y)$ as $k \rightarrow \infty$ and

$$
u_{E}(y)= \begin{cases}1 & y \in E^{1} \\ \frac{1}{2} & y \in \partial^{*} E \\ 0 & y \in E^{0}\end{cases}
$$

(iii) $\nabla u_{k} \stackrel{*}{\rightarrow} \nabla u_{E} \quad$ in $\mathcal{M}\left(\mathbb{R}^{N}\right)$;

(iv) $\left\|\nabla u_{k}\right\|(U) \rightarrow\left\|\nabla u_{E}\right\|(U)$ as $k \rightarrow \infty$, for any open set $U$ with $\left\|\nabla u_{E}\right\|(\partial U)=0$;

(v) $\nabla \chi_{E}=\nabla u_{E}$.

Proof. Only (iii) requires a proof, since (i), (ii), and (iv) are the results from the standard $B V$ theory and (v) is immediate from the definitions and the fact that $u_{E}=\chi_{E}$ almost everywhere. As for (iii), since $u_{k} \rightarrow u_{E}$ in $L^{1}\left(\mathbb{R}^{N}\right)$, then $u_{k} \rightarrow u_{E}$ when considered as distributions, which implies that $\nabla u_{k} \rightarrow \nabla u_{E}$ as distributions and consequently as measures since $\nabla u_{k}, \nabla u_{E} \in \mathcal{M}\left(\mathbb{R}^{N}\right)$. 
6.6. Remark. More generally, functions in the spaces $B V\left(\mathbb{R}^{N}\right)$ and $W^{1, p}\left(\mathbb{R}^{N}\right), 1 \leq p \leq \infty$, have precise representatives; that is, if $u \in B V\left(\mathbb{R}^{N}\right)$, then there is a function $u^{*} \in B V\left(\mathbb{R}^{N}\right)$ such that $u$ and $u^{*}$ are equal a.e. and that the mollification sequence of $u, u_{k}$, converges to $u^{*}$ at all points except those that belong to an exceptional set $E$ with $\mathcal{H}^{N-1}(E)=0$. However, this is not the same as saying that $u$ has a Lebesgue point, which is slightly stronger. A similar statement is true for functions in the Sobolev space $W^{1, p}\left(\mathbb{R}^{N}\right), 1<p \leq \infty$, except that the exceptional set $E$ has $\gamma_{p}$-capacity zero, see Definition 8.2 below. As we will see, the $\gamma_{1}$-capacity vanishes precisely on sets of $\mathcal{H}^{N-1}$ measure zero. Thus, we can say that functions in the spaces $B V$ and $W^{1, p}$ have precise representatives that are defined, respectively, $\gamma_{1}$ and $\gamma_{p}$ almost everywhere.

The next result affirms the notion that the mollification is generally a norm reducing operation.

7.1. Lemma. Let $E$ be a set of finite perimeter and let $u_{k}$ denote the mollification of $\chi_{E}$. Then

$$
\left\|\nabla u_{k}\right\|_{1} \leq\left\|\nabla \chi_{E}\right\|
$$

Proof. For any $f \in B V\left(\mathbb{R}^{N}\right)$, consider the convolutions $f_{\varepsilon}(y)=\int_{\mathbb{R}^{N}} \rho_{\varepsilon}(y-x) f(x) d x$. Using $\nabla f_{\varepsilon}=$ $\rho_{\varepsilon} * \nabla f$ and $f_{\varepsilon} \in C^{\infty}\left(\mathbb{R}^{N}\right)$, we obtain

$$
\nabla f_{\varepsilon}(y)=\int_{\mathbb{R}^{N}} \rho_{\varepsilon}(y-x) d m(x)
$$

where $m:=\nabla f$ is the measure. Thus, we have

$$
\left|\nabla f_{\varepsilon}(y)\right| \leq \int_{\mathbb{R}^{N}} \rho_{\varepsilon}(y-x) d\|m\|(x) .
$$

In particular, when $f=\chi_{E}$ and $f_{\varepsilon_{k}}=u_{k}$ with $\varepsilon_{k}=1 / k$, then $m=\nabla \chi_{E}$ and

$$
\left|\nabla u_{k}(y)\right| \leq \int_{\mathbb{R}^{N}} \rho_{\varepsilon_{k}}(y-x) d\|m\|(x) \quad \text { for all } y \in \mathbb{R}^{N}
$$

That is,

$$
\begin{aligned}
\int_{\mathbb{R}^{N}}\left|\nabla u_{k}(y)\right| d y & \leq \int_{\mathbb{R}^{N}} \int_{\mathbb{R}^{N}} \rho_{\varepsilon}(y-x) d\|m\|(x) d y \\
& =\int_{\mathbb{R}^{N}} \int_{\mathbb{R}^{N}} \rho_{\varepsilon}(y-x) d y d\|m\|(x) \leq\|m\|\left(\mathbb{R}^{N}\right) .
\end{aligned}
$$

We recall that the $B V$ space, the space of functions of bounded variation, in fact represents equivalence classes of functions so that, when a function in a class is changed on a set of $\mathcal{L}^{N_{-}}$ measure zero, it remains in this class. The same is true for sets of finite perimeter because, by definition, the characteristic function $\chi_{E}$ of a set of finite perimeter, $E$, is a function of bounded variation. Thus, it follows that $E$ may be altered by a set of $\mathcal{L}^{N}$-measure zero and still determine the same essential boundary $\partial^{m} E$. Throughout, we will choose a preferred representative for $E$ and thereby adopt the following convention.

7.2. Definition. $E:=\{y: D(E, y)=1\} \cup \partial^{m} E$.

7.3. Definition. A vector field $\boldsymbol{F} \in L^{p}\left(\Omega ; \mathbb{R}^{N}\right), 1 \leq p \leq \infty$, is called a divergence-measure field, written as $\boldsymbol{F} \in \mathcal{D M}^{p}(\Omega)$, if $\mu:=\operatorname{div} \boldsymbol{F}$ is a (signed) Radon measure with finite total variation on $\Omega$ in the sense of distributions. Thus, for $\varphi \in C_{c}^{\infty}(\Omega)$, we have

$$
\mu(\varphi):=\operatorname{div} \boldsymbol{F}(\varphi)=-\int_{\Omega} \boldsymbol{F} \cdot \nabla \varphi d y
$$

The total variation of $\mu$ is a positive measure which, for any open set $W$, is defined as

$$
\begin{aligned}
\|\mu\|(W): & =\sup \left\{\mu(\varphi):\|\varphi\|_{0 ; \Omega} \leq 1, \varphi \in C_{c}^{\infty}(W)\right\} \\
& =\sup \left\{\int_{\Omega} \boldsymbol{F} \cdot \nabla \varphi d y:\|\varphi\|_{0 ; \Omega} \leq 1, \varphi \in C_{c}^{\infty}(W)\right\} .
\end{aligned}
$$

$A$ vector field $\boldsymbol{F} \in L_{\text {loc }}^{p}(\Omega)$ means that, for any $K \Subset \Omega, \boldsymbol{F} \in \mathcal{D M}^{p}(K)$. 
8.0. Definition. Let $\boldsymbol{F} \in \mathcal{D} \mathcal{M}^{p}(\Omega), 1 \leq p \leq \infty$. For an arbitrary measurable set $E \Subset \Omega$, the trace of the normal component of $\boldsymbol{F}$ on $\partial E$ is a functional defined by

$$
(T \boldsymbol{F})_{\partial E}(\varphi)=\int_{E} \nabla \varphi \cdot \boldsymbol{F} d y+\int_{E} \varphi d \mu
$$

for all test functions $\varphi \in C_{c}^{\infty}(\Omega)$. Clearly, $(T \boldsymbol{F})_{\partial E}$ is a distribution defined on $\Omega$. Note that this definition assumes only that the set $E$ is measurable. Later, we will provide an alternative definition when $E$ is a set of finite perimeter (see Theorem 25.1).

8.1. Proposition. Let $E \Subset \Omega$ be an open set. Then $\operatorname{spt}\left((T \boldsymbol{F})_{\partial E}\right) \subset \partial E$. That is, if $\psi$ and $\varphi$ are test functions in $\mathcal{D}(\Omega)$ with $\psi=\varphi$ on $\partial E$, then $(T \boldsymbol{F})_{\partial E}(\psi)=(T \boldsymbol{F})_{\partial E}(\varphi)$.

Proof. If the support were not contained in $\partial E$, there would be a point $x_{0} \notin \partial E$ with $x_{0} \in$ $\operatorname{spt}\left((T \boldsymbol{F})_{\partial E}\right) \cap E$. This implies that, for each open set $U$ containing $x_{0}$, there exists a test function $\varphi \in C_{c}^{\infty}(U \cap E)$ such that $(T \boldsymbol{F})_{\partial E}(\varphi) \neq 0$. Choose $U$ so that $U \subset \mathbb{R}^{N} \backslash \partial E$. Let $\boldsymbol{F}_{\varepsilon}$ denote the mollification of $\boldsymbol{F}$ (see Lemma 6.4). Then, since $\operatorname{spt}\left(\boldsymbol{F}_{\varepsilon} \varphi\right) \Subset E$,

$$
\begin{aligned}
0 & =\int_{E} \operatorname{div}\left(\boldsymbol{F}_{\varepsilon} \varphi\right) d y=\left(T \boldsymbol{F}_{\varepsilon}\right)_{\partial E}(\varphi) \\
& =\int_{E} \boldsymbol{F}_{\varepsilon} \cdot \nabla \varphi+\int_{E} \varphi \operatorname{div} \boldsymbol{F}_{\varepsilon} d y \\
& \rightarrow \int_{E} \boldsymbol{F} \cdot \nabla \varphi d y+\int_{E} \varphi d \mu=(T \boldsymbol{F})_{\partial E}(\varphi) \neq 0,
\end{aligned}
$$

where we used $\partial E \cap \operatorname{spt}(\varphi)=\emptyset$ in the limit. Thus, we arrive at our desired contradiction.

8.2. Definition. For $1 \leq p \leq N$, the $p$-capacity of an arbitrary set $A \Subset \mathbb{R}^{N}$ is defined as

$$
\gamma_{p}(A):=\inf \left\{\int_{\Omega}|\nabla \varphi|^{p} d y\right\},
$$

where the infimum is taken over all test functions $\varphi \in C_{c}^{\infty}(\Omega)$ that are identically one in a neighborhood of $A$. It is well known (cf. [FZ73a]) that $\gamma_{p}(A)=0$ for $1<p<N$ implies that $\mathcal{H}^{N-p+\varepsilon}(A)=0$ for each $\varepsilon>0$ and that, conversely, if $\mathcal{H}^{N-p}(A)<\infty$, then $\gamma_{p}(A)=0$. In view of Remark 6.6 and Lemma 9.2, it is easy to verify that the class of competing functions in (8.2) can be enlarged to the Sobolev space $W^{1, p}(\Omega)$.

8.3. Remark. The case of $p=1$ requires special consideration. In 1957, Fleming conjectured that $\gamma_{1}(A)=0$ if and only if $\mathcal{H}^{N-1}(A)=0$. This was settled in the affirmative by Gustin Gus60] who proved the boxing inequality, from which Fleming's conjecture easily follows (cf. [Fle60]).

The next result is basic (cf. [Zie89, Zie69, Zie70]).

8.4. Proposition. Let $\gamma_{p}$ be the p-capacity defined as in the previous definition. Then

(i) If $E_{k} \subset \mathbb{R}^{N}$ is a sequence of arbitrary sets, then

$$
\gamma_{p}\left(\liminf _{k \rightarrow \infty} E_{k}\right) \leq \liminf _{k \rightarrow \infty} \gamma_{p}\left(E_{k}\right)
$$

(ii) If $E_{1} \subset E_{2} \subset \cdots$ are arbitrary sets, then

$$
\gamma_{p}\left(\bigcup_{k=1}^{\infty} E_{k}\right)=\lim _{k \rightarrow \infty} \gamma_{p}\left(E_{k}\right)
$$

(iii) If $K_{1} \supset K_{2} \supset \cdots$ are compact sets, then

$$
\gamma_{p}\left(\bigcap_{k=1}^{\infty} K_{k}\right)=\lim _{k \rightarrow \infty} \gamma_{p}\left(K_{k}\right)
$$


(iv) If $\left\{E_{k}\right\}$ is a sequence of Borel sets, then

$$
\gamma_{p}\left(\bigcup_{k=1}^{\infty} E_{k}\right) \leq \lim _{k \rightarrow \infty} \gamma_{p}\left(E_{k}\right)
$$

(v) If $A \subset \mathbb{R}^{N}$ is a Suslin set, then

$$
\sup \left\{\gamma_{p}(K): K^{\text {compact }} \subset A\right\}=\inf \left\{\gamma_{p}(U): U^{\text {open }} \supset A\right\} .
$$

Any set function, $\gamma$, satisfying conditions (i)-(iv) is called a true capacity in the sense of Choquet and a set $A$ satisfying condition ( $\mathrm{v}$ ) is said to be $\gamma$-capacitable.

9.1. Remark. One of the main reasons for studying the capacity is its important role in the development of Sobolev theory. It was first shown in FZ73a] that every function $u \in W^{1, p}(\Omega)$ has a Lebesgue point $\gamma_{p}$-a.e. In particular, in view of Remark 8.3, this implies that a function $u \in W^{1,1}(\Omega)$ has a Lebesgue point everywhere except for an exceptional set $E$ with $\mathcal{H}^{N-1}(E)=0$. In case $u \in B V(\Omega)$, we have a slightly weaker statement than the corresponding one for $u \in W^{1, p}(\Omega)$ :

$$
\lim _{r \rightarrow 0} f_{B(x, r)} u(y) d y=u(x) \quad \text { for } \mathcal{H}^{N-1} \text {-a.e. } x \in \Omega .
$$

It turns out that the Sobolev space is the perfect functional completion of the space $C_{c}^{\infty}(\Omega)$ relative to the p-capacity. See AS56a where the concept of perfect functional completion was initiated and developed.

9.2. Lemma. If $\boldsymbol{F} \in \mathcal{D} \mathcal{M}_{\text {loc }}^{p}\left(\mathbb{R}^{N}\right), 1 \leq p \leq \infty$, then $\|\operatorname{div} \boldsymbol{F}\|(B)=0$ whenever $B \Subset \Omega$ is a Borel set with $\gamma_{q}(B)=0, q:=p /(p-1)$. In particular, when $p=\infty$ (i.e. $\left.q=1\right)$, then $\|\operatorname{div} \boldsymbol{F}\|(B)=0$.

Proof. Because of the inner regularity of $\gamma_{q}$ and condition (v) of Proposition 8.4 it suffices to show that $\mu(K)=0$ for any compact set $K \subset B$, where $\mu:=\operatorname{div} \boldsymbol{F}$. Since $\gamma_{q}(K)=0$, then there exists a sequence of test functions $\varphi_{k} \in C_{c}^{\infty}(\Omega)$ such that

(i) $\varphi_{k}=1$ on $K$;

(ii) $\left\|\nabla \varphi_{k}\right\|_{q} \rightarrow 0$

(iii) $\varphi_{k}(y) \rightarrow 0$ for all $y \in \Omega$ except those in some set $A \subset \Omega$ with $\gamma_{q}(A)=0$.

To see that such a sequence $\varphi_{k}$ exists, we proceed as follows:

Case 1: $q>1$. Since $\gamma_{q}(K)=0$, we may choose $u_{i} \in C_{c}^{\infty}(\Omega)$ with $u_{i}=1$ near $K$ and $\left\|\nabla u_{i}\right\|_{q} \rightarrow 0$. Then Sobolev's inequality implies

$$
\left\|u_{i}\right\|_{r} \leq C\left\|\nabla u_{i}\right\|_{q} \quad \text { for } r=N q / N-q .
$$

Thus, it follows that, for a subsequence, $u_{i}(x) \rightarrow 0$ for a.e. $x$. In fact, an application of Mazur's theorem shows that the space of convex combinations of $W^{1, p}(\Omega$ is strongly closed and therefore, for a suitable subsequence, the sequence of finite convex combinations of the $u_{i}$, say $v_{k}:=\sum a_{i} u_{i}$ with $\sum_{i=1}^{k_{i}} a_{i}=1$, it follows that $v_{k}(x) \rightarrow 0$ for all $x$ except those in an exceptional set $A$ where $\gamma_{q}(A)=0$ (see [FZ73a], p. 156). Taking $\varphi_{i}:=v_{i}$ for this subsequence establishes a sequence satisfying conditions (i)-(iii) above.

Case 2: $q=1(p=\infty)$. Then the argument is modified by considering the following variational problem:

$$
\Gamma_{k, q}(K):=\inf \left\{\int_{U_{k}}\left|\nabla u_{k}\right|^{q} d y: u_{k} \in W^{1, q}\left(\mathbb{R}^{N}\right), u_{k}=1 \text { on } \overline{U_{k}}, \operatorname{spt}\left(u_{k}\right) \subset W_{k}\right\} .
$$

Let $W_{k} \supset \overline{U_{k}} \supset U_{k} \cdots \supset K$ with $U_{k}$ open and

$$
\gamma_{p}\left(\overline{W_{k}}\right)=\gamma_{p}\left(W_{k}\right)<\varepsilon_{k} \rightarrow 0 .
$$


We will also assume $W_{k}$ to be nested: $W_{1} \supset W_{2} \cdots$. For each $k$, let $\overline{W_{k}}$ be a smoothly bounded set to find that $\bigcap \overline{W_{k}}=K$. Consequently, we may take $\varphi_{k}$ to be a suitably small mollification of $\chi_{W_{k}}$ to see that conditions (i)-(iii) above are satisfied.

To prove our lemma, it suffices to show that, if $K$ is any compact set satisfying $\gamma_{q}(K)=0$, then $\mu(K)=0$. Since

(a) $\varphi_{k}=1$ on $K$

(b) $\varphi_{k} \rightarrow 0 \quad \gamma_{q^{-a} \text {-e.; }}$

(c) $\left\|\nabla \varphi_{k}\right\|_{q ; \mathbb{R}^{N}} \rightarrow 0$;

(d) $\|\mu\|(W)=\sup \left\{\int_{W}|\boldsymbol{F} \cdot \nabla \varphi| d y: \varphi \in C_{c}^{\infty}(W),|\varphi| \leq 1\right\} \quad$ for any open set $W \Subset \Omega$,

it follows that

$$
\begin{aligned}
\lim _{k \rightarrow \infty}\left(\int_{K} \varphi_{k} d \mu+\int_{U_{k} \backslash K} \varphi_{k} d \mu\right) & =\mu(K)+\lim _{k \rightarrow \infty} \int_{U_{k} \backslash K} \varphi_{k} d \mu \\
& \leq \mu(K)+\lim _{k \rightarrow \infty} \int_{U_{k} \backslash K} \varphi_{k} d\|\mu\| \\
& \leq \lim _{k \rightarrow \infty}\left|\int_{\Omega} \varphi_{k} \operatorname{div} \boldsymbol{F}\right| \leq \lim _{k \rightarrow \infty} \int_{\Omega}\left|\boldsymbol{F} \cdot \nabla \varphi_{k}\right| d x \\
& \leq\|\boldsymbol{F}\|_{p} \lim _{k \rightarrow \infty}\left\|\nabla \varphi_{k}\right\|_{q} \leq\|\boldsymbol{F}\|_{p} \cdot 0
\end{aligned}
$$

thus obtaining $\mu(K)=0$, as desired.

10.1. Corollary. If $\boldsymbol{F} \in \mathcal{D M}_{\text {loc }}^{p}(\Omega)$ for $1<p \leq N$ with $\operatorname{div} \boldsymbol{F}=: \mu$ and if $\mathcal{H}^{N-q}(A)<\infty$ for $A \Subset \Omega$ with $q=p /(p-1)$, then $\gamma_{q}(A)=0$ and hence $\|\mu\|(A)=0$.

10.2. Remark. If $\boldsymbol{F} \in \mathcal{D} \mathcal{M}^{p}\left(\mathbb{R}^{N}\right), 1 \leq p \leq \infty$, with $\operatorname{div} \boldsymbol{F}=\mu$. Then, in view of the fact that $\varphi$ is defined $\mathcal{H}^{N-q}$-a.e. and therefore $\mu$-a.e., with $\varphi \in W^{1, q}\left(\mathbb{R}^{N}\right)$, it follows that the integral

$$
\int_{\mathbb{R}^{N}} \varphi d \mu
$$

is defined and is meaningful.

10.3. Example (Chen-Frid [CF99a]). Denote $U$ the open unit square in $\mathbb{R}^{2}$ that has one of its sides contained in the line segment

$$
L:=\left\{y=\left(y_{1}, y_{2}\right): y_{1}=y_{2}\right\} \cap \partial U .
$$

Define a field $\boldsymbol{F}: \mathbb{R}^{2} \backslash L \rightarrow \mathbb{R}^{2}$ by

$$
\boldsymbol{F}(y)=\boldsymbol{F}\left(y_{1}, y_{2}\right)=\left(\sin \left(\frac{1}{y_{1}-y_{2}}\right),-\sin \left(\frac{1}{y_{1}-y_{2}}\right)\right) .
$$

Clearly, $\boldsymbol{F} \in L^{\infty}\left(\mathbb{R}^{2}\right)$, and a simple calculation reveals that $\operatorname{div} \boldsymbol{F}=0$ in $\mathbb{R}^{N} \backslash L$. Then $\boldsymbol{F}$ belongs to $\mathcal{D} \mathcal{M}^{\infty}\left(\mathbb{R}^{2}\right)$; but the field is singular on one side, $L$, of $\partial U$ and therefore, $\boldsymbol{F}$ is undefined on $\partial U$; it has no trace on $\partial U$ in the classical sense. Note also that the points of $L$ are all essential singularities of $\boldsymbol{F}$ because the following limit does not exist:

$$
\lim _{y \rightarrow x} \boldsymbol{F}(y) \quad \text { for } y \in \mathbb{R}^{2} \backslash L, x \in L,
$$

and therefore the normal trace of $\boldsymbol{F}$ on $\partial U$ is given by

$$
\lim _{t \rightarrow 0} \int_{\partial U_{t}} \boldsymbol{F}(y) \cdot \boldsymbol{\nu}(y) d \mathcal{H}^{1}(y)=\lim _{t \rightarrow 0} \int_{U_{t}} \operatorname{div} \boldsymbol{F} d y=\lim _{t \rightarrow 0} 0=0,
$$

where $\left.U_{t}:=\{y \in U: \boldsymbol{d}(y, \partial U)>t)\right\}$. Thus, we have shown the following:

(i) $\boldsymbol{F}$ is an element of $\mathcal{D} \mathcal{M}^{\infty}\left(\mathbb{R}^{2}\right)$, while each component function of $\boldsymbol{F}$ is not in $B V\left(\mathbb{R}^{2}\right)$;

(ii) $\boldsymbol{F}$ has an essential singularity at each point of $L$ and therefore cannot be defined on $L$; 
(iii) As we will see later, Theorem 25.1 $\boldsymbol{F}$ has a weak normal trace on $L$ which is sufficient for the Gauss-Green theorem to hold.

For more properties of the spaces $\mathcal{D M}^{p}$ of divergence-measure vector fields, see Chen-Frid CF99a, CF03.

The following theorem provides a product rule for the case $p=\infty$. For the sake of completeness, we will include its proof, which is slightly different from that given in CF99a. We denote by $\left\{g_{k}\right\}$ a sequence of $C_{c}^{\infty}\left(\mathbb{R}^{N}\right)$ mollifications with the property that $g_{k} \rightarrow g$ in $L^{1}\left(\mathbb{R}^{N}\right)$ and such that $\left\|\nabla g_{k}\right\| \rightarrow\|\nabla g\|$ (cf. [Zie89, p.500).

11.1. Theorem (Chen-Frid CF99a]). Let $\boldsymbol{F} \in \mathcal{D} \mathcal{M}^{\infty}\left(\mathbb{R}^{N}\right)$ and $g \in B V\left(\mathbb{R}^{N}\right)$. Then

$$
\operatorname{div}(g \boldsymbol{F})=g^{*} \operatorname{div} \boldsymbol{F}+\overline{F \cdot \nabla g},
$$

where $\overline{F \cdot \nabla g}$ denotes the weak ${ }^{*}$ limit of the measures $\boldsymbol{F} \cdot \nabla g_{k}$ and $g^{*}$ denotes the limit of the mollifiers of $g$ ( $c f$. Zie89]).

Proof. Let $\boldsymbol{F}_{\varepsilon}$ be the mollification of $\boldsymbol{F}$ and set $\mu:=\operatorname{div} \boldsymbol{F}$. Since $\boldsymbol{F}_{\varepsilon}$ are smooth, the classical product rule yields

$$
\operatorname{div}\left(g_{k} \boldsymbol{F}_{\varepsilon}\right)=g_{k} \operatorname{div} \boldsymbol{F}_{\varepsilon}+\boldsymbol{F}_{\varepsilon} \cdot \nabla g_{k} .
$$

First, we note that $\operatorname{div} \boldsymbol{F}_{\varepsilon}=(\operatorname{div} \boldsymbol{F})_{\varepsilon}=\mu_{\varepsilon} \stackrel{*}{\rightarrow} \mu$ in $\mathcal{M}\left(\mathbb{R}^{N}\right)$ as $\varepsilon \rightarrow 0$. Since $g_{k} \boldsymbol{F}_{\varepsilon} \rightarrow g_{k} \boldsymbol{F}$ in $L_{l o c}^{1}\left(\mathbb{R}^{N}\right)$ as $\varepsilon \rightarrow 0$, we obtain from (11.2) that, in the sense of distributions,

$$
\boldsymbol{F} \cdot \nabla g_{k}=\operatorname{div}\left(g_{k} \boldsymbol{F}\right)-\mu\left(g_{k}\right) .
$$

Owing to the fact that $\boldsymbol{F} \in L^{\infty}$, we see that $\boldsymbol{F} \cdot \nabla g_{k}$ is a bounded sequence in $L^{1}\left(\mathbb{R}^{N}\right)$ and hence there is a subsequence such that $\boldsymbol{F} \cdot \nabla g_{k}$ converges weak ${ }^{*}$ to some measure, denoted by $\overline{\boldsymbol{F} \cdot \nabla g}$. Letting $k \rightarrow \infty$ in (11.3) yields

$$
\overline{\boldsymbol{F} \cdot \nabla g}=\operatorname{div}(g \boldsymbol{F})-\mu\left(g^{*}\right) .
$$

The next result, Federer's coarea formula, will be of critical importance to us in the sequel.

11.2. Theorem (Fed69, Theorem 3.1). Suppose $X$ and $Y$ are Riemannian manifolds of dimension $N$ and $k$ respectively, with $N \geq k$. If $f: X \rightarrow Y$ is a Lipschitz map, then

$$
\int_{X} g(y) J f(y) d \mathcal{H}^{N}(y)=\int_{\mathbb{R}^{k}}\left\{\int_{f^{-1}(x)} g(y) d \mathcal{H}^{N-k}(y)\right\} d \mathcal{H}^{k}(x)
$$

whenever $g: X \rightarrow \mathbb{R}$ is $\mathcal{H}^{N}$-integrable. Here, $J f(y)$ denotes the $k$-dimensional Jacobian of $f$ at $y$, namely, the norm of the differential of $f$ at $y, d f(y)$. Alternatively, it is the square root of the sum of the squares of the determinants of the $k \times k$ minors of the differential of $f$ at $y$.

11.3. Lemma. Let $u: \mathbb{R}^{N} \rightarrow \mathbb{R}$ be a Lipschitz function and let $A \subset \mathbb{R}^{N}$ be a set of measure zero. Then

$$
\mathcal{H}^{N-1}\left(u^{-1}(s) \cap A\right)=0 \quad \text { for almost all } s .
$$

This can be directly seen from the coarea formula:

$$
0=\int_{A}|\nabla u(y)| d y=\int_{\mathbb{R}} \mathcal{H}^{N-1}\left(A \cap u^{-1}(s)\right) d s .
$$

One of the fundamental results of geometric measure theory is that any set of finite perimeter possesses a measure-theoretic interior normal which is suitably general to ensure the validity of the Gauss-Green theorem. 
11.4. Theorem (DeGiorgi-Federer DG61a, DG61b, Fed45, Fed58]). If E has finite perimeter, then, with $\boldsymbol{\nu}$ denoting the interior unit normal,

$$
\int_{E} \operatorname{div} \boldsymbol{F} d y=-\int_{\partial^{*} E} \boldsymbol{F}(y) \cdot \boldsymbol{\nu}(y) d \mathcal{H}^{N-1}(y)
$$

whenever $\boldsymbol{F}: \mathbb{R}^{N} \rightarrow \mathbb{R}^{N}$ is Lipschitz.

The DeGiorgi-Federer result shows that integration by parts holds on a very large and rich family of sets, but only for fields, $\boldsymbol{F}$, that are Lipschitz. As we explained in the introduction, the GaussGreen formula for $B V$ vector fields over sets of finite perimeter was treated by Maz'ja BM69 and Volpert Vol67a]. We contrast their work with that of the following result by Fuglede.

12.1. Theorem (Fuglede Fug60]). Let $\boldsymbol{F} \in \mathcal{D M}^{p}\left(\mathbb{R}^{N}\right), 1 \leq p \leq \infty$. Then there exists a function $g: \mathbb{R}^{N} \rightarrow \mathbb{R}$ with $g \in L^{q}, \frac{1}{q}+\frac{1}{p}=1$, such that

$$
\int_{E} \operatorname{div} \boldsymbol{F}=-\int_{\partial^{*} E} \boldsymbol{F}(y) \cdot \boldsymbol{\nu}(y) d \mathcal{H}^{N-1}(y)
$$

for all sets of finite perimeter, E, except possibly those for which

$$
\int_{\partial^{*} E} g(y) d \mathcal{H}^{N-1}(y)=\infty
$$

The following, which is a direct consequence of Fuglede's result, will be of great use to us. Suppose that $u: \mathbb{R}^{N} \rightarrow \mathbb{R}$ is Lipschitz. For $s<t$, consider the "annulus" $\mathcal{A}_{s ; t}:=\{x: s<u(s) \leq t\}$ determined by $u$. Then, by appealing to the coarea formula, we see that $\mathcal{A}_{s ; t}$ is a set finite perimeter for almost all $s<t$. Moreover, again appealing to the coarea formula, we see that, for almost all $s<t$

$$
(\operatorname{div} \boldsymbol{F})\left(A_{s ; t}\right):=\int_{\mathcal{A}_{s ; t}} \operatorname{div} \boldsymbol{F}=-\int_{\partial^{*} \mathcal{A}_{s ; t}} \boldsymbol{F}(y) \cdot \boldsymbol{\nu}(y) d \mathcal{H}^{N-1}(y) .
$$

One of the main objectives of this paper is to demonstrate that, when $\boldsymbol{F} \in \mathcal{D} \mathcal{M}^{\infty}\left(\mathbb{R}^{N}\right)$, we can extend Fuglede's result by showing that (12.1) and (12.2) hold for all sets of finite perimeter, not merely for "almost all" sets in the sense of Fuglede Fug60. Although we don't employ Fuglede's theorem directly, his result provided the motivation and insight for the development of our method.

The Gauss-Green formula for bounded divergence-measure fields over sets of finite perimeter was first obtained in Chen-Torres [CT05. The product rule from Lemma 11.1] was used to prove that

$$
(\operatorname{div} \boldsymbol{F})\left(E^{1}\right):=\int_{E^{1}} \operatorname{div} \boldsymbol{F}=-\int_{\partial^{*} E} 2 \overline{\chi_{E} \boldsymbol{F} \cdot \nabla u_{E}},
$$

where $2 \overline{\chi_{E} \boldsymbol{F} \cdot \nabla u_{E}}$ is the weak* limit of the measures $2 \chi_{E} \boldsymbol{F} \cdot \nabla u_{k}$. One of the main objectives of this paper is to obtain the trace measure as the limit of normal traces over smooth boundaries that approximate $\partial^{*} E$.

\section{The Normal Trace and the Gauss-Green Formula for $\mathcal{D} \mathcal{M}^{\infty}$ fields over SMOOTHLY BOUNDED SETS}

In this section we develop a method of obtaining the normal trace and thereby obtain the GaussGreen formula for a bounded divergence-measure field over any smoothly bounded set. This provides the foundation for the development for the general case.

12.2. Definition. Given a compact $C^{1}$-manifold, $M$, we define the exterior determined by $M$ to be that (connected) component, $\mathcal{U}$, of $\mathbb{R}^{N} \backslash M$ which is unbounded. The interior determined by $M$, $U$, is defined to be everything else in the complement of $M$; namely,

$$
U=\bigcup_{k=1}^{\infty} B_{k}, \quad B_{k} \subset \mathbb{R}^{N} \backslash M \text { a bounded component. }
$$


Thus,

$$
\mathbb{R}^{N} \backslash M=\mathcal{U} \cup\left(\bigcup_{k=1}^{\infty} B_{k}\right)=\mathcal{U} \cup U
$$

13.1. Theorem. Let $U \subset \mathbb{R}^{N}$ be the interior determined by a compact, $C^{1}$, manifold $M$ of dimension $N-1$ with $\mathcal{H}^{N-1}(M)<\infty$. Then, for any $\boldsymbol{F} \in \mathcal{D} \mathcal{M}_{\text {loc }}^{\infty}\left(\mathbb{R}^{N}\right)$, there exist a signed measure $\sigma$ supported on $\partial U=M$ such that $\sigma<<\mathcal{H}^{N-1}\left\llcorner\partial U\right.$ and a function $\mathcal{F}_{i} \cdot \boldsymbol{\nu}: \partial U \rightarrow \mathbb{R}$ such that

$$
\mu(U):=(\operatorname{div} \boldsymbol{F})(U)=-\sigma(\partial U)=-\int_{\partial U}\left(\mathcal{F}_{i} \cdot \boldsymbol{\nu}\right)(y) d \mathcal{H}^{N-1}(y)
$$

and

$$
\left\|\mathcal{F}_{i} \cdot \boldsymbol{\nu}\right\|_{\infty} \leq C\|\boldsymbol{F}\|_{\infty},
$$

where $C$ is a constant depending only on $N$ and $U$.

With $\boldsymbol{\nu}(y)$ denoting the interior unit normal to $M$ at $y$, we may regard $\mathcal{F}_{i} \cdot \boldsymbol{\nu}$ as the interior normal trace of $\boldsymbol{F}$ on $\partial U$ and thus write

$$
\left(\mathcal{F}_{i} \cdot \boldsymbol{\nu}\right)(y)=\boldsymbol{F}(y) \cdot \boldsymbol{\nu}(y) .
$$

Hence, with this convention, it is convenient to abuse the notation and thus write (13.1) as

$$
\mu(U)=\int_{U} \operatorname{div} \boldsymbol{F}=-\int_{\partial U} \boldsymbol{F}(y) \cdot \boldsymbol{\nu}(y) d \mathcal{H}^{N-1}(y),
$$

while bearing in mind that, since $\boldsymbol{F}$ is merely a measurable field and thus defined only up to a Lebesgue null set, it may not even be defined on $\partial U$. We use the term "interior normal trace" to suggest that $\mathcal{F}_{i} \cdot \boldsymbol{\nu}$ is determined by the behavior of $\boldsymbol{F}$ in the interior determined by the manifold $M$. The proof will reveal that, in a similar way, it is possible to define the concept of "exterior normal trace". This will be discussed more fully below in Theorem 21.1

The next example shows that our general trace theorem remains valid even though the field $\boldsymbol{F}$ is singular on an open subset of $\partial U$.

13.2. Lemma. Let $\boldsymbol{F} \in \mathcal{D} \mathcal{M}_{\text {loc }}^{\infty}\left(\mathbb{R}^{N}\right)$ whose distributional divergence is a measure $\mu$, and let $\boldsymbol{F}_{\varepsilon}$ be a mollification of $\boldsymbol{F}$. Then, because $\boldsymbol{F}_{\varepsilon}$ is smooth (in particular, Lipschitz), the classical divergence theorem holds whenever $E \Subset \mathbb{R}^{N}$ is a set of finite perimeter, namely,

$$
\int_{E} \operatorname{div} \boldsymbol{F}_{\varepsilon}=-\int_{\partial^{*} E} \boldsymbol{F}_{\varepsilon}(y) \cdot \boldsymbol{\nu}(y) d \mathcal{H}^{N-1}(y) .
$$

If, in addition, we assume the following two conditions:

(i) $\boldsymbol{F}_{\varepsilon} \rightarrow \boldsymbol{F} \mathcal{H}^{N-1}$-a.e. on $\partial^{*} E$,

(ii) $\mu(\partial E)=0$,

then

$$
\mu(E)=-\int_{\partial^{*} E} \boldsymbol{F}(y) \cdot \boldsymbol{\nu}(y) d \mathcal{H}^{N-1}(y) .
$$

The importance of this result is that, with assumptions (i) and (ii), we obtain the Gauss-Green theorem for all sets of finite perimeter whenever $\boldsymbol{F}$ is a bounded, measurable vector field with $\operatorname{div} \boldsymbol{F}=\mu$. As stated earlier, our main objective is to obtain the same result without assuming (i) and (ii), by defining a suitable notion of normal trace for $\boldsymbol{F}$ on $\partial^{*} E$.

Proof. Since $\mu_{\varepsilon}:=\operatorname{div} \boldsymbol{F}_{\varepsilon} \stackrel{*}{\rightarrow} \operatorname{div} \boldsymbol{F}=\mu$ in $\mathcal{M}\left(\mathbb{R}^{N}\right)$ and using the fact that $\boldsymbol{F}_{\varepsilon} \mathbf{L} \partial^{*} E$ is uniformly bounded, we obtain from (13.3) that

$$
\begin{aligned}
& \mu_{\varepsilon}(E):=\int_{E} \operatorname{div} \boldsymbol{F}_{\varepsilon}=-\int_{\partial^{*} E} \boldsymbol{F}_{\varepsilon}(y) \cdot \boldsymbol{\nu}(y) d \mathcal{H}^{N-1}(y) \rightarrow-\int_{\partial^{*} E} \boldsymbol{F}(y) \cdot \boldsymbol{\nu}(y) d \mathcal{H}^{N-1}(y), \\
& \mu_{\varepsilon}(E) \rightarrow \mu(E) \quad \text { (by assumption (ii)). }
\end{aligned}
$$


This establishes our result.

Proof of Theorem 13.1. According to [Whi57, there exist a $C^{1}$ unit vector field $V$ defined on $\partial U$ and a number $\delta>0$ such that $V$ is close to the interior normal on $\partial U$ and that, with the line segment joining $p \in \partial U$ and $q \in U$ defined as

$$
\lambda(p):=\{q: q=t V(p)+(1-t) p, 0 \leq t \leq 1\}
$$

then

$$
\Lambda^{*}(p):=\lambda(p) \cap B(p, \delta) \in U
$$

We think of $\Lambda^{*}(p)$ as a quasi-normal to $\partial U$ at $p$. Moreover, as $p$ ranges over $\partial U$, the quasi-normals, $\Lambda^{*}(p)$, fill out a neighborhood $U^{*}$ of $\partial U$ in a one-to-one way. That is,

$$
U^{*}=\bigcup_{p \in \partial U} \Lambda^{*}(p) \quad \text { with } \Lambda^{*}(p) \cap \Lambda^{*}(q)=\emptyset \text { when } p \neq q \mathbb{1}
$$

The mapping $\pi: U^{*} \rightarrow \partial U$, which can be considered as the projection of $U^{*}$ onto $\partial U$ along the quasi-normal, $\Lambda^{*}$, defined by

$$
\pi(q)=p \quad \text { if } q \in \Lambda_{p}^{*}
$$

is clearly of class $C^{1}$ and thus, so is the mapping $\psi: U^{*} \rightarrow \mathbb{R}$ defined by

$$
\psi(q):=|q-p| \quad \text { where } \pi(q)=p .
$$

Consequently, Sard's theorem implies that the critical values, $\mathbb{V}$, of $\psi$ are of measure zero. Thus, the implicit function theorem implies that $\partial U_{t}:=\psi^{-1}(t)$ is a $C^{1}$ manifold for almost all $t \in(0, \delta) \backslash \mathbb{V}$. The manifold $\partial U_{t}$ can be considered as a deformation of $\partial U$ along the line segments $\lambda(p)$. Thus, the sets $U_{t}:=\{\psi>t\}$ are open subsets of $U$ with smooth boundaries and $\partial U_{t}=\psi^{-1}(t)$ for a.e. $t$. Observe that $\psi^{-1}(t)=\{q \in U: q=t(V(p)-p), p \in \partial U\}$. Since $V \in C^{1}$ and $\partial U$ is compact, it follows that $V$ is Lipschitz with Lipschitz constant, say $C_{c}$, and therefore that

$$
\mathcal{H}^{N-1}\left(\psi^{-1}(t)\right) \leq C_{c} \mathcal{H}^{N-1}(\partial U)
$$

To see this, consider a local coordinate system on $\partial U$ expressed as the inverse of its projection onto the tangent plane at a point $p \in \partial U$. Consider $T_{p}(\partial U)$ as a subspace in $\mathbb{R}^{N}$ with the notation $p=\left(p^{\prime}, 0\right) \in \mathbb{R}^{N}$ as identified with $p^{\prime} \in \mathbb{R}^{N-1}$. Thus, $g: T_{p}(\partial U) \rightarrow \partial U$ will render $\partial U$ as the graph of $g$ so that $g\left(p^{\prime}\right)=\left(p^{\prime}, y\left(p^{\prime}\right)\right)$, where $y \in C^{1}$ and is the "height" function. Now define $h: \partial U \rightarrow \partial U_{t}$ by

$$
h\left(p^{\prime}, y\left(p^{\prime}\right)\right)=t V\left(p^{\prime}, y\left(p^{\prime}\right)\right)+(1-t)\left(p^{\prime}, y\left(p^{\prime}\right)\right)
$$

Clearly, all the derivatives of $h \circ g$ are uniformly bounded on compact subsets of $T_{p}(\partial U)$ and then (14.1) is evident. An application of Lemma 13.2 shows that

$$
\mu\left(U_{t}\right)=-\int_{\partial U_{t}} \boldsymbol{F}(y) \cdot \boldsymbol{\nu}(y) d \mathcal{H}^{N-1}(y) \text { for a.e. } t>0
$$

where $\mu:=\operatorname{div} \boldsymbol{F}$ is a Radon measure. Thus, the function $\boldsymbol{F} \cdot \boldsymbol{\nu} L \partial U_{t}$ induces a measure on $\mathbb{R}^{N}$. That is, for any Borel set $B \subset \mathbb{R}^{N}$, define

$$
\sigma_{t}(B):=\int_{B \cap \partial U_{t}} \boldsymbol{F}(y) \cdot \boldsymbol{\nu}(y) d \mathcal{H}^{N-1}(y) .
$$

Since $\boldsymbol{F} \in L^{\infty}$ and $\mathcal{H}^{N-1}(\partial U)<\infty$, we see from 14.1) that $\mathcal{H}^{N-1}\left(\partial U_{t}\right) \leq C$ for some $C>0$, which yields that the measures $\sigma_{t}, t>0$, form a bounded set in $\mathcal{M}\left(\mathbb{R}^{N}\right)$. Hence, there exist a sequence $\left\{t_{k}\right\} \rightarrow 0$ and Radon measures $\sigma, \sigma^{+}$, and $\sigma^{-}$with $\sigma=\sigma^{+}-\sigma^{-}$such that

$$
\left(\sigma_{t_{k}}^{+}, \sigma_{t_{k}}^{-}, \sigma_{t_{k}}\right) \stackrel{*}{\rightarrow}\left(\sigma^{+}, \sigma^{-}, \sigma\right) \quad \text { in } \mathcal{M}\left(\mathbb{R}^{N}\right)
$$

Now we show that $\sigma^{+}$is supported on $\partial U$. On the contrary, let $x \in \operatorname{spt}\left(\sigma^{+}\right) \backslash \partial U$ and choose $B(x, r)$ such that $B(x, r) \cap \partial U=\emptyset$. Since $x \in \operatorname{spt}\left(\sigma^{+}\right)$, there exists $\varphi \in C(B(x, r))$ such that $\int \varphi d \sigma^{+}:=\sigma^{+}(\varphi) \neq 0$. Then, since $\varphi$ is continuous, we find that $\sigma_{t}^{+}(\varphi) \rightarrow \sigma^{+}(\varphi) \neq 0$. This implies

\footnotetext{
${ }^{1}$ Here, we could have defined $E_{e}^{*}:=\left(\mathbb{R}^{N} \backslash \partial U\right) \cap E^{*}$ to develop the notion of the exterior normal
} 
that $\sigma_{t}^{+}(\varphi) \neq 0$ for all small $t>0$, which leads to a contradiction since $\partial U_{t} \cap B(x, r)=\emptyset$ and $\operatorname{spt}\left(\sigma_{t}^{+}\right) \subset \partial U_{t}$.

Clearly,

$$
\sigma_{t_{k}}^{+}\left(\partial U_{t_{k}}\right) \rightarrow \sigma^{+}(\partial U)
$$

since

$$
\liminf _{k \rightarrow \infty} \sigma_{t_{k}}^{+}\left(\partial U_{t_{k}}\right)=\liminf _{k \rightarrow \infty} \sigma_{t_{k}}^{+}\left(\mathbb{R}^{N}\right) \geq \sigma^{+}\left(\mathbb{R}^{N}\right)=\sigma^{+}(\partial U),
$$

whereas

$$
\limsup _{k \rightarrow \infty} \sigma_{t_{k}}^{+}\left(\partial U_{t_{k}}\right)=\limsup _{k \rightarrow \infty} \sigma_{t_{k}}^{+}(K) \leq \sigma^{+}(K)=\sigma^{+}(\partial U)
$$

for any closed set $K$ that contains $U$, especially when $K=\mathbb{R}^{N}$. In the same way, we prove that

$$
\sigma_{t_{k}}^{-}\left(\partial U_{t_{k}}\right) \rightarrow \sigma^{-}(\partial U)
$$

and hence

$$
\sigma_{t_{k}}\left(\partial U_{t_{k}}\right) \rightarrow \sigma(\partial U)
$$

Also note that the measure $\sigma$ is well-defined, which can be seen as follows: Let $U_{t_{k}^{\prime}}$ be another sequence of open sets with smooth boundaries defined by $U_{t_{k}^{\prime}}:=\left\{\psi>t_{k}^{\prime}\right\}$ for which Lemma 13.2 applies. Then, assuming that $t_{k}>t_{k}^{\prime}$ for all $k$, we have

$$
\begin{aligned}
\mu\left(U_{t_{k}^{\prime}} \backslash U_{t_{k}}\right) & =\int_{U_{t_{k}^{\prime}}} \operatorname{div} \boldsymbol{F}-\int_{U_{t_{k}}} \operatorname{div} \boldsymbol{F} \\
& =-\int_{\partial U_{t_{k}^{\prime}}} \boldsymbol{F}(y) \cdot \boldsymbol{\nu}(y) d \mathcal{H}^{N-1}(y)+\int_{\partial U_{t_{k}}} \boldsymbol{F}(y) \cdot \boldsymbol{\nu}(y) d \mathcal{H}^{N-1}(y) \\
& =-\sigma_{t_{k}^{\prime}}\left(\partial U_{t_{k}^{\prime}}\right)+\sigma_{t_{k}}\left(\partial U_{t_{k}}\right) .
\end{aligned}
$$

Since $U_{t_{k}^{\prime}} \backslash U_{t_{k}}$ is a monotone sequence of sets with $U_{t_{k}^{\prime}} \backslash U_{t_{k}} \rightarrow \emptyset$, it follows that $\mu\left(U_{t_{k}^{\prime}} \backslash U_{t_{k}}\right) \rightarrow 0$ and therefore that $\sigma_{t_{k}^{\prime}}\left(\partial U_{t_{k}^{\prime}}\right)-\sigma_{t_{k}}\left(\partial U_{t_{k}}\right) \rightarrow 0$, which shows that $\sigma$ is well-defined.

To show that $\sigma<<\mathcal{H}^{N-1} \mathrm{~L} \partial U$, let $A \subset \partial U$ with $\mathcal{H}^{N-1}(A)=0$. Then there exists an open set $G \Subset \mathbb{R}^{N}, G \supset A$, such that $G:=\bigcup_{k=1}^{N} B_{k}\left(r_{k}\right)$ with $\sum_{k} r_{k}^{N-1}<\varepsilon$ and $\mathcal{H}^{N-1}(G \cap \partial U)<\varepsilon$. Therefore, we have

$$
\left\|\sigma_{t}\right\|(G) \leq \int_{G \cap M_{t}}|\boldsymbol{F}(y) \cdot \boldsymbol{\nu}(y)| d \mathcal{H}^{N-1}(y) \leq\|\boldsymbol{F}\|_{L^{\infty}(G)} \mathcal{H}^{N-1}\left(G \cap M_{t}\right)<\varepsilon C\|\boldsymbol{F}\|_{L^{\infty}(G)},
$$

that is, for some $C>0,\left\|\sigma_{t}\right\|(G)<\varepsilon C\|\boldsymbol{F}\|_{L^{\infty}(G)}$, which leads to the conclusion $\|\sigma\|(A)=0$ as desired.

We note that, since the sets $U_{t_{k}}$ are increasing, we have

$$
\mu\left(U_{t_{k}}\right) \rightarrow \mu(U) .
$$

Thus, using (15.3) and (15.4) and sending $k \rightarrow \infty$ in $\mu\left(U_{t_{k}}\right)=-\sigma_{t_{k}}\left(\partial U_{t_{k}}\right)$ yield

$$
\mu(U)=-\sigma(\partial U) .
$$

15.1. Remark. From the proof above, it can be seen that, if $U$ were of class $C^{2}$, then the interior normals to $\partial U$ themselves would not intersect in a sufficiently small neighborhood of $\partial U$. 


\section{Almost One-Sided Smooth Approximation of Sets of Finite Perimeter}

We now proceed to establish a fundamental approximation theorem for a set of finite perimeter by a family of sets with smooth boundary essentially from the measure-theoretic interior of the set with respect to any Radon measure that is absolutely continuous with respect to $\mathcal{H}^{N-1}$. That is, we prove that, for any Radon measure $\mu$ on $\mathbb{R}^{N}$ such that $\mu<<\mathcal{H}^{N-1}$, the superlevel sets of the mollifications of the characteristic functions of sets of finite perimeter provide an approximation by smooth sets which are $\|\mu\|$-almost contained in the measure-theoretic interior of $E$. This allows us to employ Theorem 13.1 which, after passage to a limit, leads to our main result, Theorem 21.1

16.0. Lemma. Let $\mu$ be a Radon measure on $\mathbb{R}^{N}$ such that $\mu<<\mathcal{H}^{N-1}$. Let $E$ be a set of finite perimeter, and let $u_{k}$ be the mollification of $\chi_{E}$. Then, for any $t \in(0,1)$ and $A_{k ; t}:=\left\{y: u_{k}(y)>t\right\}$, there exist $\varepsilon=\varepsilon(t)$ and $k^{*}=k^{*}(\varepsilon, t)$ such that

(i) $\|\mu\|\left(A_{k ; t} \backslash E\right)<\varepsilon \quad$ for all $0<t<\frac{1}{2}$ and all $k \geq k^{*}$;

(ii) $\|\mu\|\left(A_{k ; t} \backslash E^{1}\right)<\varepsilon \quad$ for all $\frac{1}{2}<t<1$ and all $k \geq k^{*}$;

(iii) $\|\mu\|\left(E^{1} \backslash A_{k ; t}\right)<\varepsilon$ for all $\frac{1}{2}<t<1$ and all $k \geq k^{*}$;

(iv) $\|\mu\|\left(E \backslash A_{k ; t}\right)<\varepsilon$ for all $0<t<\frac{1}{2}$ and all $k \geq k^{*}$.

Proof. We first show (ii). With $\frac{1}{2}<t<1$, choose $0<\varepsilon<t-\frac{1}{2}$. Let $u_{k}$ denote (as usual) the mollification of $\chi_{E}$. We know that $u_{k}(y) \rightarrow u_{E}(y)$ for $\mathcal{H}^{N-1}$-a.e. $y$ and therefore the same is true for $\|\mu\|$ as well. By Egorov's theorem, for any $\varepsilon>0$, there is an open set $U_{\varepsilon}$ such that $\|\mu\|\left(U_{\varepsilon}\right)<\varepsilon$ and that $\left|u_{k}(y)-u_{E}(y)\right|<\varepsilon$ for all $y \notin U_{\varepsilon}$ and for all $k \geq k^{*}=k^{*}(\varepsilon, t)$. On $A_{k ; t} \backslash U_{\varepsilon}$, we have

$$
t<u_{k}(y) \text {. }
$$

Since $u_{k}(y)<u_{E}(y)+\varepsilon$ on $\mathbb{R}^{N} \backslash U_{\varepsilon}$, we have

$$
\frac{1}{2}<t-\varepsilon<u_{E}(y) \Longrightarrow u_{E}(y)=1 \Longrightarrow y \in E^{1} .
$$

This yields

$$
A_{k ; t} \backslash U_{\varepsilon} \subset E^{1} \Longrightarrow A_{k ; t} \backslash E^{1} \subset U_{\varepsilon} .
$$

Since $\|\mu\|\left(U_{\varepsilon}\right)<\varepsilon$, our desired result (ii) follows.

For the proof of (i), given $0<t<\frac{1}{2}$, we choose $0<\varepsilon<t$ and proceed as above.

We next show (iv). With $0<t<\frac{1}{2}$, choose $0<\varepsilon<\frac{1}{2}-t$. For all large $k$, we have $\left|u_{k}(y)-u_{E}(y)\right|<\varepsilon$ for all $y \notin U_{\varepsilon}$. Thus, on $E \backslash U_{\varepsilon}$,

$$
\frac{1}{2}-u_{k}(y) \leq u_{E}(y)-u_{k}(y)<\varepsilon \Longrightarrow u_{k}(y)>\frac{1}{2}-\varepsilon>t \quad \text { for all } y \in E \backslash U_{\varepsilon},
$$

which implies $E \backslash U_{\varepsilon} \subset A_{k ; t}$. Therefore, $E \backslash A_{k ; t} \subset U_{\varepsilon}$ and thus $\|\mu\|\left(E \backslash A_{k ; t}\right)<\varepsilon$.

For the proof of (iii), given $\frac{1}{2}<t<1$, we choose $0<\varepsilon<1-t$ and proceed as above.

16.1. Corollary. For each $0<t<\frac{1}{2}$ and $\varepsilon<\min \left\{t, \frac{1}{2}-t\right\}$, there exists $k^{*}=k^{*}(\varepsilon, t)>0$ such that

$$
\|\mu\|\left(A_{k ; t} \Delta E\right)<\varepsilon \quad \text { whenever } k \geq k^{*} .
$$

For each $\frac{1}{2}<t<1$ and $\varepsilon<\min \left\{t-\frac{1}{2}, 1-t\right\}$, there exists $k^{*}=k^{*}(\varepsilon, t)>0$ such that

$$
\|\mu\|\left(A_{k ; t} \Delta E^{1}\right)<\varepsilon \quad \text { whenever } k \geq k^{*} .
$$

16.2. Remark. In the previous result, we used open superlevel sets $A_{k ; t}:=\left\{y: u_{k}(y)>t\right\}$. However, we could have used closed superlevel sets $\bar{A}_{k ; t}:=\left\{y: u_{k}(y) \geq t\right\}$ to obtain the same result. We also note that, for an arbitrary Radon measure $\omega$, we have

$$
\omega\left(\bar{A}_{k ; t}\right)-\omega\left(A_{k ; t}\right)=\omega\left(\partial A_{k ; t}\right)=0
$$


for all but countably many $t$, for the reason that the family of sets $\left\{\partial A_{k ; t}: t \in \mathbb{R}\right\}$ is pairwise disjoint and any Radon measure $\omega$ can assign positive values to only a countable number of such a family.

17.1. Corollary. For each $t>0$, there exist $\varepsilon(t)$ and $k^{*}=k^{*}(\varepsilon, t)>0$ such that

(i) $\|\mu\|\left(A_{k ; t} \Delta E\right)=\|\mu\|\left(\bar{A}_{k ; t} \Delta E\right)<\varepsilon$ for all but countably many $t \in\left(0, \frac{1}{2}\right)$ and for $k \geq k^{*}$;

(ii) $\|\mu\|\left(A_{k ; t} \Delta E^{1}\right)=\|\mu\|\left(\bar{A}_{k ; t} \Delta E^{1}\right)<\varepsilon$ for all but countably many $t \in\left(\frac{1}{2}, 1\right)$ and for $k \geq k^{*}$;

(iii) $\|\mu\|\left(\partial A_{k ; t} \Delta E\right)=\|\mu\|\left(u_{k}^{-1}(t) \Delta E\right)<\varepsilon$ for almost all $t \in\left(0, \frac{1}{2}\right)$ and for $k \geq k^{*}$;

(iv) $\|\mu\|\left(\partial A_{k, t} \Delta E^{1}\right)=\|\mu\|\left(u_{k}^{-1}(t) \Delta E^{1}\right)<\varepsilon$ for almost all $t \in\left(\frac{1}{2}, 1\right)$ and for $k \geq k^{*}$.

For the case $t=\frac{1}{2}$, only (i) and (iii) in Lemma 15.2 remain valid. To see this, we first show

17.2. Lemma. Let $\mu$ be a Radon measure on $\mathbb{R}^{N}$ such that $\mu<<\mathcal{H}^{N-1}$. Let $E$ be a set of finite perimeter, and let $u_{k}$ be the mollification of $\chi_{E}$. Then, for $t=\frac{1}{2}$ and $\varepsilon>0$, there exists $k^{*}=k^{*}(\varepsilon)$ such that

$$
\|\mu\|\left(E^{1} \backslash A_{k ; \frac{1}{2}}\right)<\varepsilon \quad \text { and } \quad\|\mu\|\left(A_{k ; \frac{1}{2}} \backslash E\right)<\varepsilon .
$$

Proof. Since $u_{k}(y) \rightarrow u_{E}(y)$ for $\mathcal{H}^{N-1}$-a.e. $y$, the dominated convergence theorem implies that $u_{k} \rightarrow u_{E}$ in $L^{1}\left(\mathbb{R}^{N},\|\mu\|\right)$. Thus, given any $\varepsilon>0$, for $k$ large enough, we have

$$
\frac{\varepsilon}{2} \geq \int_{\mathbb{R}^{N}}\left|u_{E}-u_{k}\right| d\|\mu\| \geq \int_{E^{1} \backslash A_{k ; \frac{1}{2}}}\left(u_{E}-u_{k}\right) d\|\mu\| \geq\left(1-\frac{1}{2}\right)\|\mu\|\left(E^{1} \backslash A_{k ; \frac{1}{2}}\right),
$$

which implies

$$
\|\mu\|\left(E^{1} \backslash A_{k ; \frac{1}{2}}\right) \leq \varepsilon
$$

In the same way, we compute

$$
\frac{\varepsilon}{2} \geq \int_{A_{k ; \frac{1}{2}} \backslash E}\left|u_{E}-u_{k}\right| d\|\mu\| \geq\left(\frac{1}{2}-0\right)\|\mu\|\left(A_{k ; \frac{1}{2}} \backslash E\right),
$$

which implies

$$
\|\mu\|\left(A_{k ; \frac{1}{2}} \backslash E\right) \leq \varepsilon
$$

The following remark shows that, with $t=\frac{1}{2}$ and with $\mu=\mathcal{H}^{N-1}\left\llcorner\partial^{*} E \geq 0\right.$, (ii) and (iv) in Lemma 15.2 do not hold.

17.3. Remark. If we define $E:=\left\{y \in \mathbb{R}^{N}:|y| \leq 1\right\}$, then $u_{k}^{-1}\left(\frac{1}{2}\right) \subset \mathbb{R}^{N} \backslash E$ for all $k$, and therefore it is clear that

$$
\mathcal{H}^{N-1}\left(\left(A_{k ; \frac{1}{2}} \backslash E^{1}\right) \cap \partial^{*} E\right)=\mathcal{H}^{N-1}\left(\partial^{*} E\right) \nrightarrow 0 \quad \text { as } k \rightarrow \infty .
$$

If we now define $E:=\left\{y \in \mathbb{R}^{N}:|y| \geq 1\right\}$, then $u_{k}^{-1}\left(\frac{1}{2}\right) \subset E$ for all $k$ and thus

$$
\mathcal{H}^{N-1}\left(\left(E \backslash A_{k ; \frac{1}{2}}\right) \cap \partial^{*} E\right)=\mathcal{H}^{N-1}\left(\partial^{*} E\right) \nrightarrow 0 \quad \text { as } k \rightarrow \infty .
$$

17.4. Lemma. There exists a number $0<C<\infty$ such that, for all positive integers $k$ and almost all $t \in(0,1)$,

$$
\mathcal{H}^{N-1}\left(u_{k}^{-1}(t)\right) \leq C .
$$

Proof. From Corollary 16.1, it follows that, for almost all $t \in(0,1)$, there exists a sequence of smoothly bounded sets $A_{k ; t}$ such that either $\chi_{A_{k ; t}} \rightarrow \chi_{E^{1}} \mu$-a.e. (if $\frac{1}{2}<t<1$ ), or $\chi_{A_{k ; t}} \rightarrow \chi_{E} \mu$-a.e. (if $0<t<\frac{1}{2}$ ). Since $\mu=\mathcal{H}^{N-1}\left\llcorner\partial^{*} E<<\mathcal{H}^{N-1}\right.$, it follows that $\chi_{A_{k \cdot t}} \rightarrow \chi_{E}$ everywhere except for a set of Lebesgue measure zero. Since these functions are integrable, we may consider these 
functions as distributions and thus, by appealing to Lebesgue's dominated convergence theorem, we may conclude

$$
\chi_{A_{k ; t}} \rightarrow \chi_{E} \quad \text { in the sense of distributions, }
$$

and therefore

$$
\nabla \chi_{A_{k ; t}} \rightarrow \nabla \chi_{E} \quad \text { in the sense of distributions. }
$$

Since all the functions are in $B V$, we find that

$$
\nabla \chi_{A_{k ; t}} \stackrel{*}{\rightarrow} \nabla \chi_{E} \quad \text { in } \mathcal{M}\left(\mathbb{R}^{N}\right),
$$

and the limit $\nabla \chi_{E}$ is independent of $t \in(0,1)$. Therefore, the uniform boundedness theorem for measures (Corollary 4.2 also Lemma 4.3 ) implies that these measures are uniformly bounded in $\mathcal{M}\left(\mathbb{R}^{N}\right)$; that is, there exists $0<C<\infty$, independent of $t$, such that, for almost all $t \in(0,1)$,

$$
\sup _{k}\left\|\nabla \chi_{A_{k ; t}}\right\|\left(\mathbb{R}^{N}\right) \leq C \text {. }
$$

Since

our result follows.

$$
\left\|\nabla \chi_{A_{k ; t}}\right\|=\mathcal{H}^{N-1}\left\llcorner u_{k}^{-1}(t),\right.
$$

We now offer another proof of this result here for the purpose of broadening the context of our development. By using the theory of integral currents [Fed69, the result is immediate. Rather than actually introducing integral currents, we will introduce a small structure that reflects the argument from integral currents. For this, let $\mathcal{V}$ denote the Banach space $C_{c}^{1}(K)$ of vector fields $\psi$ on $K$ endowed with norm

$$
\|\psi\|:=\sup _{y \in K}\left(|\psi(y)|+\sum_{i=1}^{N}\left|\nabla \psi_{i}(y)\right|\right),
$$

where $K$ is a compact set such that $E \Subset K$.

Let

$$
T_{E}(\psi):=\int_{E} \psi(y) d y
$$

and, for almost every $t \in(0,1)$, let

$$
T_{k ; t}(\psi):=\int_{\left\{y: u_{k}(y)>t\right\}} \psi(y) d y=\int_{A_{k ; t}} \psi(y) d y \quad \text { for } \psi \in \mathcal{V} .
$$

Then, for $\psi \in \mathcal{V}$, we define the linear operators:

$$
\partial T_{E}(\psi):=T_{E}(\operatorname{div} \psi)=\int_{E} \operatorname{div} \psi d y=-\int_{\partial^{*} E} \psi \cdot \boldsymbol{\nu} d \mathcal{H}^{N-1},
$$

and

$$
\partial T_{k ; t}(\psi):=T_{k ; t}(\operatorname{div} \psi)=\int_{A_{k ; t}} \operatorname{div} \psi d y=-\int_{\partial A_{k ; t}} \psi \cdot \boldsymbol{\nu} d \mathcal{H}^{N-1},
$$

where $\boldsymbol{\nu}$ is the interior unit normal.

Since $u_{k}^{-1}(t)$ is a $C^{\infty}$-manifold, then

$$
\left\|\partial T_{k ; t}\right\|:=\sup _{\|\psi\| \leq 1}\left|\partial T_{k ; t}(\psi)\right|=\mathcal{H}^{N-1}\left(u_{k}^{-1}(t)\right) .
$$

Indeed, with $\psi:=\frac{\boldsymbol{\nu}_{k}}{\left|\boldsymbol{\nu}_{k}\right|}$ defined on the manifold $u_{k}^{-1}(t)$, the norm-preserving extension of $\psi$ to all of $\mathbb{R}^{N}$ by Whitney's extension theorem yields the inequality

$$
\left\|\partial T_{k ; t}\right\|:=\sup _{\|\psi\| \leq 1}\left|\partial T_{k ; t}(\psi)\right| \geq \mathcal{H}^{N-1}\left(u_{k}^{-1}(t)\right) .
$$

The opposite inequality is obvious. 
Moreover, we find by the dominated convergence theorem that

$$
\lim _{k \rightarrow \infty} \partial T_{k ; t}(\psi) \rightarrow \partial T_{E}(\psi) \quad \text { for } \psi \in \mathcal{V},
$$

and therefore,

$$
\sup _{k}\left\{\left|\partial T_{k ; t}(\psi)\right|\right\}<\infty \quad \text { for } \psi \in \mathcal{V} .
$$

By the uniform boundedness principle (Theorem 4.1), we see that, since $\partial T_{k ; t}$ is a linear functional on $\mathcal{V}$ whose week limit, $\partial T_{E}$, is independent of $t$, we have

$$
\sup _{k} \mathcal{H}^{N-1}\left(u_{k}^{-1}(t)\right)=\sup _{k}\left\|\partial T_{k ; t}\right\|<\infty
$$

which gives our desired result.

The above argument simply rephrases the following basic fact from the theory of currents. We know that, since $E$ has finite perimeter, $T_{E}$ is an integral current. Moreover, the currents $T_{k ; t}$ converge to $T_{E}$ weakly and therefore so do their boundaries, $\partial T_{k ; t} \rightarrow \partial T_{E}$; that is,

$$
\int_{u_{k}^{-1}(t)} \sigma d \mathcal{H}^{N-1}=\int_{\partial A_{k ; t}} \sigma d \mathcal{H}^{N-1} \rightarrow \int_{\partial^{*} E} \sigma d \mathcal{H}^{N-1}
$$

for each smooth differential $(N-1)$-form $\sigma$. Appealing to Corollary 4.2 yields our result.

19.1. Lemma. Let $u: \Omega \rightarrow \mathbb{R}$ be a Lipschitz function and $E \Subset \Omega$ a set of finite perimeter. Then

$$
\mathcal{H}^{N-1}\left(\partial^{*} E \cap u^{-1}(t)\right)=0 \text { for almost all } t .
$$

Proof. This result follows directly from Lemma 11.3. since we know that $\mathcal{H}^{N-1}\left(\partial^{*} E\right)<\infty$ for any bounded set of finite perimeter, $E \subset \mathbb{R}^{N}$.

19.2. Lemma. For almost every $\frac{1}{2}<t<1$, we have

$$
\mathcal{H}^{N-1}\left(\partial^{*} E \cap u_{k}^{-1}(t)\right)=0
$$

and

$$
\lim _{k \rightarrow \infty} \mathcal{H}^{N-1}\left(\partial^{*} E \cap A_{k ; t}\right)=0 .
$$

Proof. This can be seen as follows. If we use Corollary 16.1 with $\mu=\mathcal{H}^{N-1} \mathrm{~L} \partial^{*} E$, we obtain

$$
\lim _{k \rightarrow \infty} \mu\left(A_{k ; t} \backslash E^{1}\right)=\lim _{k \rightarrow \infty} \mathcal{H}^{N-1}\left(A_{k ; t} \cap \partial^{*} E\right)=0 .
$$

Clearly, (19.1) follows from Lemma 19.1 (see also Remark 16.2).

19.3. Theorem (Approximation theorem). For almost every $\frac{1}{2}<t<1$, we have

$$
\lim _{k \rightarrow \infty} \mathcal{H}^{N-1}\left(\left(E^{0} \cup \partial^{*} E\right) \cap u_{k}^{-1}(t)\right)=0 .
$$

Proof. Since the Lebesgue measure is absolutely continuous with respect to $\mathcal{H}^{N-1}$, then using (16.2) in Corollary 16.1 with $s>\frac{1}{2}$ leads to

$$
\left|A_{k ; s} \Delta E^{1}\right| \rightarrow 0 \quad \text { as } k \rightarrow \infty .
$$

Therefore, since

$$
R_{k ; s}:=A_{k ; s} \backslash E^{1}=A_{k ; s} \cap\left(E^{0} \cup \partial^{*} E^{0}\right),
$$

it follows that

$$
\left|R_{k ; s}\right| \rightarrow 0 \quad \text { provided that } s>\frac{1}{2} .
$$


Remark 16.2 indicates that we have the option of defining $A_{k ; t}:=\left\{y: u_{k}(y) \geq t\right\}$ without altering the development. With this option in force, we have $u_{k}^{-1}(s) \subset A_{k ; s}$ and consequently, by the coarea formula (Theorem[11.2),

$$
\begin{aligned}
\int_{R_{k ; s}}\left|\nabla u_{k}\right| d y & =\int_{0}^{1} \mathcal{H}^{N-1}\left(u_{k}^{-1}(t) \cap R_{k ; s}\right) d t \\
& =\int_{s}^{1} \mathcal{H}^{N-1}\left(u_{k}^{-1}(t) \cap\left(E^{0} \cup \partial^{*} E^{0}\right)\right) d t .
\end{aligned}
$$

Since $\nabla u_{k} \stackrel{*}{\rightarrow} \nabla \chi_{E}$ and $\left\|\nabla u_{k}\right\|_{1} \leq\left\|\nabla \chi_{E}\right\|$ (Lemma 7.1), it follows from Vitali's convergence theorem for $s>\frac{1}{2}$ that

$$
\int_{R_{k ; s}}\left|\nabla u_{k}\right| d y \rightarrow 0
$$

Thus, for a subsequence, we can conclude that, for a.e. $t>s$,

$$
\mathcal{H}^{N-1}\left(u_{k}^{-1}(t) \cap\left(E^{0} \cup \partial^{*} E^{0}\right)\right) \rightarrow 0 \quad \text { as } k \rightarrow \infty .
$$

The dependence on the subsequence is illusory. The reason is that, if there were a subsequence such that, for a.e. $t$,

$$
\mathcal{H}^{N-1}\left(u_{k}^{-1}(t) \cap\left(E^{0} \cup \partial^{*} E^{0}\right)\right) \rightarrow \alpha \neq 0 \quad \text { as } k \rightarrow \infty,
$$

then one could appeal to our previous argument to conclude that, for some further subsequence and for a.e. $t$,

$$
\mathcal{H}^{N-1}\left(u_{k}^{-1}(t) \cap\left(E^{0} \cup \partial^{*} E^{0}\right)\right) \rightarrow 0 \quad \text { as } k \rightarrow \infty,
$$

which is contrary to our assertion that $\alpha \neq 0$.

Since $s>\frac{1}{2}$ was fixed arbitrarily at the beginning of the proof, we conclude that, for a.e. $t>\frac{1}{2}$,

$$
\mathcal{H}^{N-1}\left(u_{k}^{-1}(t) \cap\left(E^{0} \cup \partial^{*} E^{0}\right)\right) \rightarrow 0 \quad \text { as } k \rightarrow \infty .
$$

\section{MAin TheOREM}

In this section we establish our main result, Theorem 21.1 Let $\boldsymbol{F} \in \mathcal{D M}_{\text {loc }}^{\infty}(\Omega)$. We define a measure $\sigma_{k ; t}$ for all Borel sets $B \Subset \Omega$ by

$$
\sigma_{k ; t}(B):=\int_{B \cap \partial A_{k ; t}} \boldsymbol{F}(y) \cdot \boldsymbol{\nu}(y) d \mathcal{H}^{N-1}(y),
$$

where $\boldsymbol{F}(y) \cdot \boldsymbol{\nu}(y)$ denotes the normal trace of $\boldsymbol{F}$ on the smooth boundary $\partial A_{k ; t}$ introduced in Lemma 15.2 .

We begin with a lemma that will lead to several of the assertions in Theorem 21.1.

20.1. Lemma. If $E \Subset \Omega$ is an arbitrary set of finite perimeter, then we have

$$
\int_{E} \boldsymbol{F} \cdot \nabla u_{k} d y=\int_{0}^{1} \int_{E \cap u_{k}^{-1}(t)} \boldsymbol{F} \cdot \boldsymbol{\nu}_{k} d \mathcal{H}^{N-1} d t
$$

for any $\boldsymbol{F} \in L_{l o c}^{\infty}\left(\Omega ; \mathbb{R}^{N}\right)$, where $u_{k}$ denotes the mollification of $\chi_{E}$ as introduced in Definition 6.3 and Lemma 6.5.

Proof. Let $\mathcal{N}$ be the set on which $\nabla u_{k}=0$. Then

$$
\begin{aligned}
\int_{E} \boldsymbol{F} \cdot \nabla u_{k} d y & =\int_{E \backslash \mathcal{N}} \boldsymbol{F} \cdot \nabla u_{k} d y+\int_{\mathcal{N}} \boldsymbol{F} \cdot \nabla u_{k} d y \\
& =\int_{E \backslash \mathcal{N}}\left|\nabla u_{k}\right| \frac{\boldsymbol{F} \cdot \nabla u_{k}}{\left|\nabla u_{k}\right|} d y+0 \\
& =\int_{E}\left|\nabla u_{k}\right| g d y,
\end{aligned}
$$


where $g=\frac{\chi_{E \backslash \mathcal{N}} \boldsymbol{F} \cdot \nabla u_{k}}{\left|\nabla u_{k}\right|}$. Then, by the Coarea Formula, we have

$$
\begin{aligned}
\int_{E} \boldsymbol{F} \cdot \nabla u_{k} d y & =\int_{0}^{1} \int_{u_{k}^{-1}(t) \cap(E \backslash \mathcal{N})} g d \mathcal{H}^{N-1} d t \\
& =\int_{0}^{1} \int_{u_{k}^{-1}(t) \cap(E \backslash \mathcal{N})} \boldsymbol{F} \cdot \boldsymbol{\nu}_{k} d \mathcal{H}^{N-1} d t \\
& =\int_{0}^{1} \int_{u_{k}^{-1}(t) \cap E} \boldsymbol{F} \cdot \boldsymbol{\nu}_{k} d \mathcal{H}^{N-1} d t
\end{aligned}
$$

where we used $\boldsymbol{\nu}_{k}(y)=\frac{\nabla u_{k}(y)}{\left|\nabla u_{k}(y)\right|}$ for $y \in u_{k}^{-1}(t) \cap(E \backslash \mathcal{N})$.

With the help of Theorem 13.1 and the results in $\S 4$, we now establish our main theorem.

21.1. Theorem (Main theorem). Let $\Omega \subset \mathbb{R}^{N}$ be an open set. Suppose that $\boldsymbol{F} \in \mathcal{D M}_{\text {loc }}^{\infty}(\Omega)$ with $\operatorname{div} \boldsymbol{F}=\mu \in \mathcal{M}(\Omega)$. Let $E \Subset \Omega$ be a set of finite perimeter. Then

(I) For almost every $s \in\left(\frac{1}{2}, 1\right)$, there exist a signed measure $\sigma_{i}$ (independent of $s$ ) and a family of sets $A_{k ; s}$ with smooth boundaries such that

(a) $\|\mu\|\left(A_{k ; s} \Delta E^{1}\right) \rightarrow 0$;

(b) The measure $\sigma_{i}$ is the weak ${ }^{*}$ limit of the measures $\sigma_{k ; s}$;

(c) $\sigma_{i}$ is carried by $\partial^{*} E$ in the sense that $\left\|\sigma_{i}\right\|\left(\Omega \backslash \partial^{*} E\right)=0$;

(d) $\left\|\sigma_{i}\right\|<<\mathcal{H}^{N-1} \mathrm{~L} \partial^{*} E$;

(e) $\lim _{k \rightarrow \infty} \mathcal{H}^{N-1}\left(\partial A_{k ; s} \cap\left(E^{0} \cup \partial^{*} E\right)\right)=0$;

(f) $\lim _{k \rightarrow \infty}\left\|\sigma_{k ; s}\right\|\left(E^{0} \cup \partial^{*} E\right)=0$;

(g) The density of $\sigma_{i}$, denoted as $\mathcal{F}_{i} \cdot \boldsymbol{\nu}$, is called the interior normal trace relative to $E$ of $\boldsymbol{F}$ on $\partial^{*} E$ and satisfies

$$
\int_{E^{1}} \operatorname{div} \boldsymbol{F}=: \mu\left(E^{1}\right)=-\sigma_{i}\left(\partial^{*} E\right)=-\int_{\partial^{*} E}\left(\mathcal{F}_{i} \cdot \boldsymbol{\nu}\right)(y) d \mathcal{H}^{N-1}(y)
$$

(h) If $\left(2 \boldsymbol{F} \cdot \nabla u_{k}\right) \chi_{E}$ is considered as a sequence of measures, then this sequence converges weak ${ }^{*}$ to the measure $\left(\mathcal{F}_{i} \cdot \boldsymbol{\nu}\right) \mathcal{H}^{N-1}\left\llcorner\partial^{*} E\right.$, i.e.,

$$
\left(2 \boldsymbol{F} \cdot \nabla u_{k}\right) \chi_{E} \stackrel{*}{\rightarrow}\left(\mathcal{F}_{i} \cdot \boldsymbol{\nu}\right) \mathcal{H}^{N-1} \mathrm{~L} \partial^{*} E \quad \text { in } \mathcal{M}(\Omega)
$$

(i) $\left\|\sigma_{i}\right\|=\left\|\mathcal{F}_{i} \cdot \boldsymbol{\nu}\right\|_{\infty ; \partial^{*} E, \mathcal{H}^{N-1}} \leq\|F\|_{\infty ; E}$.

(II) For almost every $s \in\left(0, \frac{1}{2}\right)$, there exist a signed measure $\sigma_{e}$ (independent of $s$ ) and a family of sets $A_{k ; s}$ with smooth boundaries such that

(a) $\|\mu\|\left(A_{k ; s} \Delta E\right) \rightarrow 0$;

(b) The measure $\sigma_{e}$ is the weak ${ }^{*}$ limit of $\sigma_{k ; s}$;

(c) $\sigma_{e}$ is carried by $\partial^{*} E$ in the sense that $\left\|\sigma_{e}\right\|\left(\Omega \backslash \partial^{*} E\right)=0$;

(d) $\left\|\sigma_{e}\right\|<<\mathcal{H}^{N-1}\left\llcorner\partial^{*} E\right.$;

(e) $\lim _{k \rightarrow \infty} \mathcal{H}^{N-1}\left(\partial A_{k ; s} \cap E\right)=\lim _{k \rightarrow \infty} \mathcal{H}^{N-1}\left(u_{k}^{-1}(s) \cap E\right)=0$;

(f) $\lim _{k \rightarrow \infty}\left\|\sigma_{k ; s}\right\|\left(\Omega \backslash E^{0}\right)=\lim _{k \rightarrow \infty}\left\|\sigma_{k ; s}\right\|(E)=0$;

(g) The density of $\sigma_{e}$, denoted as $\mathcal{F}_{e} \cdot \boldsymbol{\nu}$, is called the exterior normal trace relative to $E$ of $\boldsymbol{F}$ on $\partial^{*} E$ and satisfies

$$
\int_{E} \operatorname{div} \boldsymbol{F}=: \mu(E)=-\sigma_{e}\left(\partial^{*} E\right)=-\int_{\partial^{*} E}\left(\mathcal{F}_{e} \cdot \boldsymbol{\nu}\right)(y) d \mathcal{H}^{N-1}(y) ;
$$

(h) If $\left(2 \boldsymbol{F} \cdot \nabla u_{k}\right) \chi_{E^{0}}$ is considered as a sequence of measures, then this sequence converges weak* to the measure $\left(\mathcal{F}_{e} \cdot \boldsymbol{\nu}\right) \mathcal{H}^{N-1} \mathrm{~L} \partial^{*} E$, i.e.,

$$
\left(2 \boldsymbol{F} \cdot \nabla u_{k}\right) \chi_{E^{0}} \stackrel{*}{\rightarrow}\left(\mathcal{F}_{e} \cdot \boldsymbol{\nu}\right) \mathcal{H}^{N-1}\left\llcorner\partial^{*} E \quad \text { in } \mathcal{M}(\Omega) ;\right.
$$


(i) $\left\|\sigma_{e}\right\|=\left\|\mathcal{F}_{e} \cdot \boldsymbol{\nu}\right\|_{\infty ; \partial^{*} E, \mathcal{H}^{N-1}} \leq\|\boldsymbol{F}\|_{\infty ; \Omega \backslash E} \cdot$

Proof. We will prove only part (I), since the proof of part (II) is virtually identical. For notational simplicity, we will use the notation $\sigma$ rather than $\sigma_{i}$ in the proof of part (I). Throughout the proof, we will consider only those values of $s \in\left(\frac{1}{2}, 1\right)$ for which the results in $\S 4$ are valid for all the mollified functions, $u_{k}$, of $\chi_{E}$ thus omitting at most a set of measure zero. Without increasing the measure of this exceptional set, call it $\mathcal{S}$, we will also include those values of $s$ for which $\|\mu\|\left(u_{k}^{-1}(s)\right) \neq 0$ for all $k$. For the rest of the proof, we fix such an $s \notin \mathcal{S}$.

We start with (a). We consider the sets $A_{k ; s}$ as in Lemma 15.2. The desired result follows directly from Corollary 16.1 .

(b) Since $\boldsymbol{F}$ is bounded, Lemma 17.4 implies that there exists a constant $C$ such that

$$
\left\|\sigma_{k ; s}\right\|(\Omega) \leq C,
$$

which yields, as in (14.2), the existence of a signed measure $\sigma_{s}$ such that

$$
\sigma_{k ; s} \stackrel{*}{\rightarrow} \sigma_{s} \text { in } \mathcal{M}(\Omega) .
$$

Utilizing (16.2), we also obtain that $\mu\left(A_{k ; s}\right) \rightarrow \mu\left(E^{1}\right)$. Since Theorem 13.1 yields $\mu\left(A_{k ; s}\right)=$ $-\sigma_{k ; s}(\Omega)$, we obtain, after letting $k \rightarrow \infty$,

$$
\mu\left(E^{1}\right)=-\sigma_{s}(\Omega) .
$$

Since the left side of equation (22.3) is independent of $s$, we show next that $\sigma_{s}$ is also independent of $s$ (and independent of the sequence in the weak* convergence (22.2)). To see this, we fix any $\phi \in C_{c}^{1}(\Omega)$ and note that, since $\boldsymbol{F}$ is a divergence-measure field, the product rule in Lemma 11.1 implies that $\phi \boldsymbol{F}$ is also a divergence-measure field. Proceeding as above with $\phi \boldsymbol{F}$ instead of $\boldsymbol{F}$, we obtain

$$
\int_{E^{1}} \operatorname{div}(\phi \boldsymbol{F})=-\int_{\Omega} \phi d \sigma_{s}
$$

for any $\phi \in C_{c}^{1}(\Omega)$. Therefore, for any two measures $\sigma_{s}$ and $\sigma_{s^{\prime}}$ that are the limits in (22.2), we have that $\int_{\Omega} \phi d \sigma_{s}=\int_{\Omega} \phi d \sigma_{s^{\prime}}$ for any $\phi \in C_{c}^{1}(\Omega)$ and thus we conclude that $\sigma_{s}=\sigma_{s^{\prime}}$.

(c) Let $A \subset \Omega \backslash \partial^{*} E$ be an arbitrary Borel set. Referring to (5.4), we see that

$$
\left\|\nabla \chi_{E}\right\|(A)=0 .
$$

On the other hand, we know

$$
\begin{aligned}
0 & =\left\|\nabla \chi_{E}\right\|(A) \\
& =\inf \left\{\left\|\nabla \chi_{E}\right\|(U): A \subset U, U \text { open }\right\} \\
& =\inf \left\{\left\|\nabla \chi_{E}\right\|(U): A \subset U, U \text { open, }\left\|\nabla \chi_{E}\right\|(\partial U)=0\right\} .
\end{aligned}
$$

In order to prove that $\|\sigma\|(A)=0$, we proceed by contradiction via assuming $\|\sigma\|(A)>0$. From (22.5), there is an open set $U \supset A$ such that $\left\|\nabla \chi_{E}\right\|(\partial U)=0$ and

$$
\left\|\nabla \chi_{E}\right\|(U)<\frac{\|\sigma\|(A)}{2\|\boldsymbol{F}\|_{\infty}} .
$$

From Lemma 20.1, we have

$$
\int_{U}\left|\boldsymbol{F} \cdot \nabla u_{k}\right| d y=\int_{0}^{1} \int_{U \cap u_{k}^{-1}(t)}\left|\boldsymbol{F} \cdot \boldsymbol{\nu}_{k}\right| d \mathcal{H}^{N-1} d t .
$$


Since $U$ is open and $\sigma_{k ; t} \stackrel{*}{\rightarrow} \sigma$ in $\mathcal{M}(\Omega)$,

$$
\begin{aligned}
\|\sigma\|(A) & \leq 2 \int_{\frac{1}{2}}^{1}\|\sigma\|(U) d t \leq 2 \int_{\frac{1}{2}}^{1} \liminf _{k \rightarrow \infty}\left\|\sigma_{k ; t}\right\|(U) d t \\
& \leq 2 \int_{0}^{1} \liminf _{k \rightarrow \infty}\left\|\sigma_{k ; t}\right\|(U) d t \leq 2 \liminf _{k \rightarrow \infty} \int_{0}^{1}\left\|\sigma_{k ; t}\right\|(U) d t
\end{aligned}
$$

by Fatou's lemma. Therefore, we have

$$
\begin{aligned}
\|\sigma\|(A) & \leq 2 \liminf _{k \rightarrow \infty} \int_{0}^{1} \int_{u_{k}^{-1}(t) \cap U}|\boldsymbol{F}(y) \cdot \boldsymbol{\nu}(y)| d \mathcal{H}^{N-1}(y) d t \\
& =2 \liminf _{k \rightarrow \infty} \int_{U}\left|\boldsymbol{F} \cdot \nabla u_{k}\right| d y \\
& \leq 2\|\boldsymbol{F}\|_{\infty} \lim _{k \rightarrow \infty} \int_{U}\left|\nabla u_{k}\right| d y \\
& =2\|\boldsymbol{F}\|_{\infty}\left\|\nabla \chi_{E}\right\|(U) \\
& <\|\sigma\|(A),
\end{aligned}
$$

where we used Lemma 6.5 (iv) and the fact that $\left\|\nabla \chi_{E}\right\|(\partial U)=0$. This yields a contradiction and thus establishes our result.

(d) Let $A \subset \Omega$ be a Borel set with $\mathcal{H}^{N-1}(A)=0$. Then, appealing to (5.4), we find that $\left\|\nabla \chi_{E}\right\|(\Omega)=0$. From this, the proof can be proceeded precisely as in (c) to yield our desired conclusion.

(e) This is the result of Theorem 19.3.

(f) In view of the definition

$$
\sigma_{k ; s}(B):=\int_{\partial A_{k ; s} \cap B} \boldsymbol{F}(y) \cdot \boldsymbol{\nu}(y) d \mathcal{H}^{N-1}(y),
$$

and the fact that the normal trace is bounded, the result follows immediately from (e).

(g) From (a), we have the existence of smoothly bounded sets such that

$$
\|\mu\|\left(A_{k ; s} \Delta E^{1}\right) \rightarrow 0 \quad \text { as } k \rightarrow \infty
$$

where $s>1 / 2$ was fixed at the beginning of the proof. From Theorem 13.1, we know that our desired result holds for smoothly bounded sets:

$$
\mu\left(A_{k ; s}\right):=\int_{A_{k ; s}} \operatorname{div} \boldsymbol{F}=-\int_{\partial A_{k ; s}} \boldsymbol{F}(y) \cdot \boldsymbol{\nu}(y) d \mathcal{H}^{N-1}(y) .
$$

We note that, with our notation in force, we may write (23.2) as

$$
\mu\left(A_{k ; s}\right)=-\sigma_{k ; s}(\Omega)=-\sigma_{k ; s}\left(\partial A_{k ; s}\right) .
$$

Since

$$
\mu\left(A_{k ; s}\right) \rightarrow \mu\left(E^{1}\right) \quad \text { and } \quad \sigma_{k ; s}(\Omega) \rightarrow \sigma(\Omega) \quad \text { as } k \rightarrow \infty,
$$

we obtain

$$
\mu\left(E^{1}\right)=-\sigma\left(\partial^{*} E\right)
$$

Because $\|\sigma\|<<\mathcal{H}^{N-1}\left\llcorner\partial^{*} E\right.$, we know that there exists $\mathcal{F}_{i} \cdot \boldsymbol{\nu} \in L^{1}\left(\partial^{*} E\right)$ such that

$$
\sigma(B)=\int_{B \cap \partial^{*} E}\left(\mathcal{F}_{i} \cdot \boldsymbol{\nu}\right)(y) d \mathcal{H}^{N-1}(y)
$$

which gives (21.1). 
(h) From Lemma 20.1, we obtain

$$
\lim _{k \rightarrow \infty} \int_{E} \boldsymbol{F} \cdot \nabla u_{k} d y=\lim _{k \rightarrow \infty} \int_{0}^{1} \int_{u_{k}^{-1}(t) \cap E} \boldsymbol{F}(y) \cdot \boldsymbol{\nu}_{k}(y) d \mathcal{H}^{N-1}(y) d t=\lim _{k \rightarrow \infty} \int_{0}^{1} \sigma_{k ; t}(E) d t .
$$

Thus,

$$
\begin{aligned}
\overline{\chi_{E} \boldsymbol{F} \cdot \nabla u_{E}}(\Omega) & :=\lim _{k \rightarrow \infty} \int_{\Omega} \chi_{E} \boldsymbol{F} \cdot \nabla u_{k} d y \\
& =\lim _{k \rightarrow \infty} \int_{E} \boldsymbol{F} \cdot \nabla u_{k} d y \\
& =\lim _{k \rightarrow \infty} \int_{\frac{1}{2}}^{1} \sigma_{k ; t}(E) d t+\lim _{k \rightarrow \infty} \int_{\frac{1}{2}}^{1} \sigma_{k ; t}\left(E^{0}\right) d t \quad \text { (by (f) above) } \\
& =\lim _{k \rightarrow \infty} \int_{\frac{1}{2}}^{1} \sigma_{k ; t}(\Omega) d t \\
& =\frac{1}{2} \sigma(\Omega) .
\end{aligned}
$$

Let $\varphi$ be a function in $C_{c}^{1}(\Omega)$. Since $\varphi \boldsymbol{F}$ is also a bounded divergence-measure field, we can proceed as above with the vector field $\varphi \boldsymbol{F}$ instead of $\boldsymbol{F}$ to conclude that

$$
\int_{\Omega} \varphi d \overline{\chi_{E} \boldsymbol{F} \cdot \nabla u_{E}}=\frac{1}{2} \int_{\Omega} \varphi d \sigma
$$

which implies that $\sigma=2 \overline{\chi_{E} \boldsymbol{F} \cdot \nabla u_{E}}$.

(i) We have that, for $\mathcal{H}^{N-1}$-a.e. $y \in \partial^{*} E$,

$$
\mathcal{F}_{i} \cdot \boldsymbol{\nu}(y)=\lim _{r \rightarrow 0} \frac{\sigma(B(y, r))}{\left\|\nabla \chi_{E}\right\|(B(y, r))},
$$

where we can choose the balls $B(y, r)$ such that $\left\|\nabla \chi_{E}\right\|(\partial B(y, r))=\|\sigma\|(\partial B(y, r))=0$.

Using a similar argument as in (c), we obtain

$$
\begin{aligned}
\|\sigma\|(B(y, r)) & =2 \int_{\frac{1}{2}}^{1}\|\sigma\|(B(y, r)) d t \\
& =2 \lim _{k \rightarrow \infty} \int_{\frac{1}{2}}^{1}\left\|\sigma_{k ; t}\right\|(B(y, r)) d t \\
& =2 \lim _{k \rightarrow \infty} \int_{\frac{1}{2}}^{1}\left(\left\|\sigma_{k ; t}\right\|\left(B(y, r) \cap E^{1}\right)+\left\|\sigma_{k ; t}\right\|\left(B(y, r) \cap\left(E^{0} \cup \partial^{*} E\right)\right)\right) d t \\
& =2 \lim _{k \rightarrow \infty} \int_{\frac{1}{2}}^{1}\left\|\sigma_{k ; t}\right\|\left(B(y, r) \cap E^{1}\right) d t \\
& =2 \lim _{k \rightarrow \infty} \int_{\frac{1}{2}}^{1} \int_{u_{k}^{-1}(t) \cap B(y, r) \cap E^{1}}|\boldsymbol{F} \cdot \boldsymbol{\nu}| d \mathcal{H}^{N-1} d t \\
& \leq 2\|\boldsymbol{F}\|_{\infty ; E^{1}} \lim _{k \rightarrow \infty} \int_{\frac{1}{2}}^{1} \int_{u_{k}^{-1}(t) \cap B(y, r)} d \mathcal{H}^{N-1} d t
\end{aligned}
$$

where we have used the fact that $\left\|\sigma_{k ; t}\right\|\left(E^{0} \cup \partial^{*} E\right) \rightarrow 0$ as $k \rightarrow \infty$ for a.e. $t>1 / 2$. 
Therefore, from (24.2), we obtain

$$
\begin{aligned}
\left|\mathcal{F}_{i} \cdot \boldsymbol{\nu}(y)\right| & \leq \lim _{r \rightarrow 0} \frac{\|\sigma\|(B(y, r))}{\left\|\nabla \chi_{E}\right\|(B(y, r))} \\
& \leq 2\|\boldsymbol{F}\|_{\infty ; E^{1}} \lim _{r \rightarrow 0} \lim _{k \rightarrow \infty} \frac{\int_{\frac{1}{2}}^{1} \int_{u_{k}^{-1}(t) \cap B(y, r)} d \mathcal{H}^{N-1} d t}{\int_{B(y, r)}\left|\nabla u_{k}\right|} \\
& =2\|\boldsymbol{F}\|_{\infty ; E^{1}} \lim _{r \rightarrow 0} \lim _{k \rightarrow \infty} \frac{\int_{\frac{1}{2}}^{1} \int_{u_{k}^{-1}(t) \cap B(y, r)} d \mathcal{H}^{N-1} d t}{\int_{0}^{1} \int_{u_{k}^{-1}(t) \cap B(x, r)} d \mathcal{H}^{N-1} d t} \\
& =\|\boldsymbol{F}\|_{\infty ; E^{1}}=\|\boldsymbol{F}\|_{\infty ; E} .
\end{aligned}
$$

As a direct result, we obtain the Gauss-Green theorem for divergence-measure fields over sets of finite perimeter. This also shows that our definition of the normal trace is in agreement with that given in the sense of distributions, Definition 7.3

25.1. Theorem (Gauss-Green theorem). Let $\Omega \subset \mathbb{R}^{N}$ be an open set. Let $\boldsymbol{F} \in \mathcal{D M}_{\text {loc }}^{\infty}(\Omega)$ and let $E \Subset \Omega$ be a bounded set of finite perimeter. Then,

$$
\int_{E^{1}} \varphi \operatorname{div} \boldsymbol{F}+\int_{E^{1}} \boldsymbol{F} \cdot \nabla \varphi=-\int_{\partial^{*} E} \varphi\left(\mathcal{F}_{i} \cdot \boldsymbol{\nu}\right) d \mathcal{H}^{N-1}
$$

for all $\varphi \in C_{c}^{\infty}(\Omega)$, where $\boldsymbol{\nu}$ is the measure-theoretic interior unit normal to $E$ on $\partial^{*} E$.

Proof. From Lemma 11.1, it follows that $\varphi \boldsymbol{F}$ is a bounded divergence-measure field and

$$
\operatorname{div}(\varphi \boldsymbol{F})=\varphi \operatorname{div} \boldsymbol{F}+\boldsymbol{F} \cdot \nabla \varphi .
$$

Following the proof of Theorem 21.1 applied to $\varphi \boldsymbol{F}$ (instead of $\boldsymbol{F}$ ), (see Theorem[11.1), we obtain

$$
\int_{E^{1}} \operatorname{div}(\varphi \boldsymbol{F})=-\int_{\partial^{*} E} \varphi\left(\mathcal{F}_{i} \cdot \boldsymbol{\nu}\right) d \mathcal{H}^{N-1},
$$

which, due to (25.2), gives the desired result.

We conclude this section with the following remark.

25.2. Remark. Theorem 25.1 implies that, when $E$ is an open set of finite perimeter, our trace $\mathcal{F}_{i} \cdot \boldsymbol{\nu}$ agrees with the one defined in (8.1).

\section{The Divergence-Measures of Jump Sets via the Normal Traces}

In Theorem 21.1, we have defined the interior and exterior normal traces of $\boldsymbol{F} \in \mathcal{D M}_{\text {loc }}^{\infty}(\Omega), \mathcal{F}_{i} \cdot \boldsymbol{\nu}$ and $\mathcal{F}_{e} \cdot \boldsymbol{\nu}$, over the boundary of a set of finite perimeter $E \Subset \Omega$. In order to obtain the interior normal trace of $\boldsymbol{F}$ on $\partial^{*} \tilde{E}$, where $\tilde{E}:=E^{0} \cup \partial^{m} E$, we reproduce the proof of Theorem 21.1 and apply it to $\tilde{E}$. Therefore, the trace measure, denoted by $\sigma_{-}$, is obtained by using the level sets $B_{k ; s}=\left\{v_{k}>s\right\}$ for some $s \in\left(\frac{1}{2}, 1\right)$, where $v_{k}$ is the mollification of $\chi_{\tilde{E}}$. We note that, for all $y \in \Omega$,

$$
\rho_{\varepsilon} * \chi_{E}(y)+\rho_{\varepsilon} * \chi_{\tilde{E}}(y)=1,
$$

and therefore

$$
v_{k}^{-1}(s)=u_{k}^{-1}(1-s)
$$

where $1-s \in\left(0, \frac{1}{2}\right)$. Since $-\boldsymbol{\nu}$ is the interior unit normal to $\tilde{E}$, we have

$$
\begin{aligned}
\sigma_{-}(\Omega) & =-\lim _{k \rightarrow \infty} \int_{\partial B_{k ; s}} \boldsymbol{F} \cdot \boldsymbol{\nu} d \mathcal{H}^{N-1}=-\lim _{k \rightarrow \infty} \int_{\partial A_{k ; 1-s}} \boldsymbol{F} \cdot \boldsymbol{\nu} d \mathcal{H}^{N-1} \\
& =-\lim _{k \rightarrow \infty} \sigma_{k ; 1-s}\left(\mathbb{R}^{N}\right)=-\sigma_{e}(\Omega) .
\end{aligned}
$$

The following observation now becomes evident. 
25.3. Corollary. The interior trace of $\boldsymbol{F}$ relative to $\tilde{E}$ on $\partial^{*} E$ is the same as minus the exterior trace of $\boldsymbol{F}$ relative to $E$ on $\partial^{*} E$.

In order to establish the relation between $\sigma_{i}$ and $\sigma_{e}$, we subtract (21.2) from (21.1) and obtain the following formula for $\mu=\operatorname{div} \boldsymbol{F}$.

26.1. Corollary. $\quad \mu\left(\partial^{*} E\right)=\int_{\partial^{*} E}\left(\mathcal{F}_{i} \cdot \boldsymbol{\nu}-\mathcal{F}_{e} \cdot \boldsymbol{\nu}\right)(y) d \mathcal{H}^{N-1}(y)$.

We offer the following simple example to illustrate our result. This example also dramatically demonstrates the difference between the classical derivative and the weak (distributional) derivative.

26.2. Example. Consider the most elementary situation: $N=1, \Omega:=(-1,2), E:=[0,1]$, and $f$ is a non-decreasing function defined on $(-1,2)$ which is continuous everywhere except at $y=0,1$, at which points we assume that $f$ has right-continuity.

(i) Case $\frac{1}{2}<s<1$. Since $f$ is in $B V$, we know that $f^{\prime}=\mu$ for some measure $\mu$. Then, according to Theorem 21.1.

$$
\mu\left(E^{1}\right)=\mu((0,1)):=\int_{0+}^{1-} f^{\prime}=\mathcal{F}_{i} \cdot \boldsymbol{\nu}(1)-\mathcal{F}_{i} \cdot \boldsymbol{\nu}(0),
$$

where $\mathcal{F}_{i} \cdot \boldsymbol{\nu}(1)=\lim _{y \rightarrow 1-} f(y)$ and $\mathcal{F}_{i} \cdot \boldsymbol{\nu}(0)=f(0+)$. Indeed, the sets $A_{k ; s}$, with fixed $\frac{1}{2}<s<1$, form a nested family of open intervals contained in $[0,1]$. The measures $\sigma_{k}$ correspond to $f$ evaluated on the point masses located at $y_{k}$; thus, as in (23.4), $f\left(y_{k}\right)$ converges to a limit, $\mathcal{F}_{i} \cdot \boldsymbol{\nu}(1)$.

(ii) Case $0<s<\frac{1}{2}$. Then the sets $A_{k ; s}, 0<s<\frac{1}{2}$, form a nested family of open intervals containing $[0,1]$. Similar to the above, we have

$$
\mu(E)=\mu([0,1]):=\int_{0-}^{1+} f^{\prime}=\mathcal{F}_{e} \cdot \boldsymbol{\nu}(1)-\mathcal{F}_{e} \cdot \boldsymbol{\nu}(0),
$$

the measures $\sigma_{k}$ correspond to $f$ evaluated on the point masses located at $y_{k}$, and thus $\mathcal{F}_{e} \cdot \boldsymbol{\nu}(1)=$ $f(1+)$ and $\mathcal{F}_{e} \cdot \boldsymbol{\nu}(0)=\lim _{y \rightarrow 0-} f(y)$.

\section{Consistency of the Normal Traces with the Classical Traces}

We now proceed to show the consistency of our normal traces with the classical traces when $\boldsymbol{F}$ is continuous. First we have the following lemma.

26.3. Lemma. Let $\mu=\operatorname{div} \boldsymbol{F}$ for $\boldsymbol{F} \in \mathcal{D} \mathcal{M}^{\infty}\left(\mathbb{R}^{N}\right) \cap C\left(\mathbb{R}^{N} ; \mathbb{R}^{N}\right)$. Then

$$
\|\mu\|(G)=0
$$

for any set $G$ that can be written as the graph of a Lipschitz function $f$.

Proof. First we have

$$
G:=\left\{\left(y^{\prime}, f\left(y^{\prime}\right)\right): y^{\prime} \in W \subset \mathbb{R}^{N-1}\right\} .
$$

By regularity of $\mu$, it suffices to show that $\mu(K)=0$ for any compact set $K \subset G$. Given any compact set $K \subset G$, let $U_{k} \subset \mathbb{R}^{N}$ be a sequence of open sets satisfying

$$
\mu\left(U_{k} \cap G\right) \rightarrow \mu(K) .
$$

Fix any set $U_{k}$. We note by Besicovitch's theorem that $U_{k}$ can be written up to a set of $\|\mu\|$-measure zero as a countable union of disjoint open parallelepipeds $I_{i}^{k}$ (the fact that we can use parallelepipeds instead of balls follows from Morse [Mor47]). Thus, we have

$$
\bigcup_{i=1}^{\infty} I_{i}^{k} \subset U_{k} \quad \text { and } \quad\|\mu\|\left(U_{k} \backslash \bigcup_{i=1}^{\infty} I_{i}^{k}\right)=0 .
$$

Denote $U_{k}$ simply as $U$ and $I_{i}^{k}$ as $I_{i}$. We fix an $i$ and note that, for $t$ small enough, the graphs $T_{t}:=\left\{\left(y^{\prime}, f\left(y^{\prime}\right)+t\right): y^{\prime} \in W \subset \mathbb{R}^{N-1}\right\}$ and $B_{t}:=\left\{\left(y^{\prime}, f\left(y^{\prime}\right)-t\right): y^{\prime} \in W \subset \mathbb{R}^{N-1}\right\}$ are contained 
in $I_{i}$. Let $R_{t}$ be the region inside $I_{i}$, bounded above and below by $T_{t}$ and $B_{t}$ respectively. For a.e. $t$, we define

$$
\alpha_{t}=\int_{\partial R_{t} \backslash\left(T_{t} \cup B_{t}\right)} \boldsymbol{F}(y) \cdot \boldsymbol{\nu}(y) d \mathcal{H}^{N-1}(y) \quad \text { for a.e. } t,
$$

where $\boldsymbol{\nu}(y)$ is the interior unit normal to $R_{t}$ on $\partial R_{t} \backslash\left(T_{t} \cup B_{t}\right)$. Since Lemma 13.2 applies to $R_{t}$ for a.e. $t$, we arrive at

$$
\begin{aligned}
\mu\left(R_{t}\right) & =\int_{R_{t}} \operatorname{div} \boldsymbol{F} \\
& =-\int_{B_{t}} \boldsymbol{F}(y) \cdot \boldsymbol{\nu}(y) d \mathcal{H}^{N-1}(y)-\int_{T_{t}} \boldsymbol{F}(y) \cdot \boldsymbol{\nu}(y) d \mathcal{H}^{N-1}(y)-\alpha_{t} \\
& =\int_{T_{t}} \boldsymbol{F}\left(y^{\prime}, y_{n}-2 t\right) \cdot \boldsymbol{\nu}(y) d \mathcal{H}^{N-1}(y)-\int_{T_{t}} \boldsymbol{F}\left(y^{\prime}, y_{n}\right) \cdot \boldsymbol{\nu}(y) d \mathcal{H}^{N-1}(y)-\alpha_{t} \\
& =\int_{T_{t}}\left(\boldsymbol{F}\left(y^{\prime}, y_{n}-2 t\right)-\boldsymbol{F}\left(y^{\prime}, y_{n}\right)\right) \cdot \boldsymbol{\nu}(y) d \mathcal{H}^{N-1}(y)-\alpha_{t} .
\end{aligned}
$$

Since $\boldsymbol{F}$ is continuous and $\alpha_{t} \rightarrow 0$ as $t \rightarrow 0$, we find that there exists $t_{0}(\varepsilon, F, G)>0$ such that

$$
\mu\left(R_{t}\right) \leq \varepsilon \quad \text { for all } t \leq t_{0}(\varepsilon, F, G)
$$

Then we have

$$
\mu\left(I_{i} \cap G\right)=\lim _{t \rightarrow 0} \mu\left(R_{t}\right) \leq \varepsilon,
$$

which implies $\mu\left(I_{i} \cap G\right)=0$ since $\varepsilon$ is arbitrary. Therefore, using (26.2), we obtain

$$
\mu(K)=\lim _{k \rightarrow \infty} \mu\left(U_{k} \cap G\right)=\lim _{k \rightarrow \infty} \sum \mu\left(I_{i}^{k} \cap G\right)=0 .
$$

27.1. Theorem. If $\boldsymbol{F} \in \mathcal{D M}_{\text {loc }}^{\infty}(\Omega)$ is continuous and $E \Subset \Omega$ is a set of finite perimeter, then $\sigma_{i}=\overline{F \cdot \nabla u_{E}}$, and the normal trace $\mathcal{F}_{i} \cdot \boldsymbol{\nu}$ is in fact the classical dot product $\boldsymbol{F} \cdot \boldsymbol{\nu}$, where $\boldsymbol{\nu}$ is the interior unit normal to $E$.

Proof. We recall that, by definition, $E=E^{1} \cup \partial^{*} E$. Denote $\tilde{E}=E^{0} \cup \partial^{*} E$. Then we have

$$
\begin{aligned}
\overline{\boldsymbol{F} \cdot \nabla u_{E}} & =\lim _{k \rightarrow \infty} \int_{\mathbb{R}^{N}} \boldsymbol{F} \cdot \nabla u_{k} d y \\
& =\lim _{k \rightarrow \infty} \int_{\mathbb{R}^{N}} \chi_{E} \boldsymbol{F} \cdot \nabla u_{k} d y+\lim _{k \rightarrow \infty} \int_{\mathbb{R}^{N}} \chi_{\tilde{E}} \boldsymbol{F} \cdot \nabla u_{k} d y .
\end{aligned}
$$

If $v_{k}$ denotes the convolution $\chi_{\tilde{E}} * \rho_{1 / k}$, since $u_{k}+v_{k}=1$, we obtain

$$
\begin{aligned}
\overline{\boldsymbol{F} \cdot \nabla u_{E}} & =\lim _{k \rightarrow \infty} \int_{\mathbb{R}^{N}} \chi_{E} \boldsymbol{F} \cdot \nabla u_{k} d y-\lim _{k \rightarrow \infty} \int_{\mathbb{R}^{N}} \chi_{\tilde{E}} \boldsymbol{F} \cdot \nabla v_{k} d y \\
& =\frac{\sigma_{i}}{2}+\frac{\sigma_{e}}{2}=\frac{1}{2}\left(\sigma_{i}+\sigma_{i}-\mu\left(\partial^{*} E\right)\right) \\
& =\sigma_{i}-\frac{1}{2} \mu\left(\partial^{*} E\right),
\end{aligned}
$$

where we used Theorem 21.1 (h) and Corollary 26.1.

Since $\partial^{*} E$ is an $(N-1)$-rectifiable set (see (5.5) $)$, it follows from Lemma 26.3 that

$$
\|\mu\|\left(\partial^{*} E\right)=0
$$

that is,

$$
\overline{\boldsymbol{F} \cdot \nabla u_{E}}=\sigma_{i} .
$$


Thus, for $\mathcal{H}^{N-1}$-a.e. $y \in \partial^{*} E$,

$$
\left(\mathcal{F}_{i} \cdot \boldsymbol{\nu}\right)(y)=\lim _{r \rightarrow 0} \frac{\overline{\boldsymbol{F} \cdot \nabla u_{E}}(B(y, r))}{\left\|\nabla \chi_{E}\right\|(B(y, r))}=\lim _{r \rightarrow 0} \lim _{k \rightarrow \infty} \frac{\int_{B(y, r)} \boldsymbol{F} \cdot \nabla u_{k} d x}{\int_{B(y, r)} d\left\|\nabla \chi_{E}\right\|} .
$$

Since $\nabla u_{k} \rightarrow \nabla \chi_{E}$ weak ${ }^{*}$ and $\boldsymbol{F}$ is continuous, and noting that $r_{j}$ can be chosen such that $\left\|\nabla \chi_{E}\right\|\left(\partial B\left(y, r_{j}\right)\right)=0$, we obtain

$$
\begin{aligned}
\left(\mathcal{F}_{i} \cdot \boldsymbol{\nu}\right)(y) & =\lim _{j \rightarrow \infty} \frac{\int_{B\left(y, r_{j}\right)} \boldsymbol{F} \cdot \nabla \chi_{E}}{\int_{B\left(y, r_{j}\right)} d\left\|\nabla \chi_{E}\right\|}=\lim _{j \rightarrow \infty} \frac{\int_{B\left(y, r_{j}\right)} \boldsymbol{F}(x) \cdot \boldsymbol{\nu}(x) d\left\|\nabla \chi_{E}\right\|(x)}{\int_{B\left(y, r_{j}\right)} d\left\|\nabla \chi_{E}\right\|(x)} \\
& =\boldsymbol{F}(y) \cdot \boldsymbol{\nu}(y),
\end{aligned}
$$

by differentiation of measures.

The following corollary gives more information of the trace $\sigma_{i}$ and the level sets $u_{k}^{-1}(s)$ when $s \rightarrow \frac{1}{2}+$.

28.1. Corollary. The trace measure $\sigma_{i}$ given in Theorem 21.1 satisfies

$$
\begin{aligned}
\sigma_{i}\left(\mathbb{R}^{N}\right) & =2 \lim _{k \rightarrow \infty} \int_{E} \boldsymbol{F} \cdot \nabla u_{k} d y=2 \lim _{k \rightarrow \infty} \lim _{s \rightarrow \frac{1}{2}+} \int_{A_{k ; s}} \boldsymbol{F} \cdot \nabla u_{k} d y \\
& =2 \lim _{k \rightarrow \infty} \int_{A_{k ; \frac{1}{2}}} \boldsymbol{F} \cdot \nabla u_{k} d y .
\end{aligned}
$$

Proof. Theorem 21.1 (h) shows

$$
\sigma_{i}\left(\mathbb{R}^{N}\right)=2 \lim _{k \rightarrow \infty} \int_{E} \boldsymbol{F} \cdot \nabla u_{k} d y .
$$

Using Lemma 20.1, we find

$$
\begin{aligned}
\sigma_{i}\left(\mathbb{R}^{N}\right) & =2 \lim _{s \rightarrow \frac{1}{2}+} \int_{s}^{1} \sigma_{i}\left(\mathbb{R}^{N}\right) d t=2 \lim _{s \rightarrow \frac{1}{2}+} \lim _{k \rightarrow \infty} \int_{s}^{1} \int_{u_{k}^{-1}(t)} \boldsymbol{F} \cdot \boldsymbol{\nu} d \mathcal{H}^{N-1} d t \\
& =2 \lim _{s \rightarrow \frac{1}{2}+} \lim _{k \rightarrow \infty} \int_{A_{k ; s}} \boldsymbol{F} \cdot \nabla u_{k} d y .
\end{aligned}
$$

One can easily verify that the limits $s \rightarrow \frac{1}{2}+$ and $k \rightarrow \infty$ can be interchanged. Noting that $\bigcup_{s>\frac{1}{2}} A_{k ; s}=A_{k ; \frac{1}{2}}$, we conclude

$$
\sigma_{i}\left(\mathbb{R}^{N}\right)=2 \lim _{k \rightarrow \infty} \lim _{s \rightarrow \frac{1}{2}+} \int_{A_{k ; s}} \boldsymbol{F} \cdot \nabla u_{k} d y=2 \lim _{k \rightarrow \infty} \int_{A_{k ; \frac{1}{2}}} \boldsymbol{F} \cdot \nabla u_{k} d y .
$$

\section{ONE-SIDED APPROXIMATION OF SETS OF FINITE PERIMETER}

It is well-known that, a set of finite perimeter, $E$, cannot be approximated by smooth sets that lie completely in the interior of $E$. For example, consider the open unit disk with a single radius removed, and let $U$ be the resulting open set. Then the Hausdorff measure of the boundary of $U$ is $2 \pi$ plus the measure of the radius, while the Hausdorff measure of the reduced boundary is $2 \pi$. Thus, if $U_{k}$ is an approximating open subset of $U$, then its boundary will be close to that of boundary $U$ and so its the Hausdorff measure will be close to $2 \pi$ plus 1 . Adding more radii, say $m$ of them, will force the approximating set to have boundaries whose Hausdorff measure close to $2 \pi$ plus $m$. In general, if we let $K$ denote any compact subset without interior and of infinite Hausdorff measure, then the approximating sets will have boundaries whose measures will necessarily tend to infinity. On the other hand, the one-sided approximation is possible for open sets of class $C^{1}$, see Theorem 13.1. More generally we have the following: 
28.2. Proposition. Let $U \subset \mathbb{R}^{N}$ be an open set with $\mathcal{H}^{N-1}(\partial U)<\infty$. Then there exists a sequence of bounded open sets $U_{k} \subset \overline{U_{k}} \subset U$ such that

(i) $\left|U_{k}\right|=\left|\overline{U_{k}}\right|$;

(ii) $\left|U_{k}\right| \rightarrow|U|$;

(iii) $\mathcal{H}^{N-1}\left(\partial U_{k}\right) \rightarrow \mathcal{H}^{N-1}(\partial U)$.

Proof. By definition, for each integer $k$, there exists a covering of $\partial U$ by balls

$$
\partial U \subset \cup B_{i}\left(r_{i}\right)
$$

each with radius $r_{i}$, such that

$$
\sum_{i=1}^{\infty} \mathcal{H}^{N-1}\left(\partial B_{i}\left(r_{i}\right)\right)=\sum_{i=1}^{\infty} \omega_{N-1} r_{i}^{N-1}<\mathcal{H}^{N-1}(\partial U)+\frac{1}{k}
$$

where $\omega_{N-1}$ is the $\mathcal{H}^{N-1}$ measure of the boundary of the unit ball in $\mathbb{R}^{N}$. Since $\partial \Omega$ is compact, the covering may be taken as a finite covering, say by $m$ of them, $B_{1}\left(r_{1}\right), B_{1}\left(r_{2}\right), \ldots, B_{m}\left(r_{m}\right)$. Then the open set $V_{k}:=\bigcup B_{i}\left(r_{i}\right)$ has the property that

$$
\partial V_{k} \subset \bigcup_{i=1}^{m} \partial B_{i}\left(r_{i}\right)
$$

and therefore that

$$
\mathcal{H}^{N-1}\left(\partial V_{k}\right) \leq \mathcal{H}^{N-1}\left(\bigcup_{i=1}^{m} \partial B_{i}\left(r_{i}\right)\right) \leq \sum_{i=1}^{\infty} \omega_{N-1} r_{i}^{N-1}<\mathcal{H}^{N-1}(\partial U)+\frac{1}{k}
$$

Thus, the open sets $U_{k}:=U \backslash \bar{V}_{k} \subset U$ will satisfy our desired result, except that they are not smooth.

Given an arbitrary set of finite perimeter, $E$, we know from $\S 4$ that $E$ can be approximated by sets with smooth boundaries essentially from the measure-theoretic interior of $E$, that is, a one-sided approximation can "almost" be achieved (see Theorem 21.1(e)). On the other hand, the next result shows that, if $E$ is sufficiently regular, there does, in fact, exist a one-sided approximation. The condition of regularity we impose is similar to Lewis's uniformly flat condition in potential theory Lew88a.

29.1. Theorem. Suppose that $E$ is a bounded set of finite perimeter with the property that, for all $y \in \partial E$, there are positive constants $c_{0}$ and $r_{0}$ such that

$$
\frac{\left|E^{0} \cap B(y, r)\right|}{|B(y, r)|} \geq c_{0} \quad \text { for all } r \leq r_{0} .
$$

Then there exists $t \in(0,1)$ such that

$$
A_{k ; t} \Subset E \quad \text { for large } k \text {. }
$$

Proof. Choose a mollifying kernel $\rho$ such that $\rho=1$ on $B\left(0, \frac{1}{2}\right)$. If $y \in \partial E$, we have

$$
\begin{aligned}
v_{k}(y):=\chi_{\mathbb{R}^{N} \backslash E} * \rho_{\varepsilon_{k}}(y) & =\frac{1}{\varepsilon_{k}^{N}} \int_{B\left(y, \varepsilon_{k}\right)} \chi_{\mathbb{R}^{N} \backslash E}(x) \rho\left(\frac{x-y}{\varepsilon_{k}}\right) d x \\
& \geq \frac{1}{\varepsilon_{k}^{N}} \int_{B\left(y, \frac{\varepsilon_{k}}{2}\right)} \chi_{\mathbb{R}^{N} \backslash E}(x) d x \\
& =\frac{\left|\left(\mathbb{R}^{N} \backslash E\right) \cap B\left(y, \frac{\varepsilon_{k}}{2}\right)\right|}{\varepsilon_{k}^{N}} \\
& =\frac{\left|E^{0} \cap B\left(y, \frac{\varepsilon_{k}}{2}\right)\right|}{\varepsilon_{k}^{N}} \geq c_{0} / 2^{N}:=\tilde{c}_{0},
\end{aligned}
$$


where $0<\tilde{c_{0}}<1$ depends only on the dimension $N$ and is independent of the point $y$. Note that $u_{k}(y)+v_{k}(y)=1$ for all $y \in \mathbb{R}^{N}$. Therefore, for all $y \in \partial E$,

$$
u_{k}(y)=1-v_{k}(y) \leq 1-\tilde{c_{0}} .
$$

Thus, taking $1-\tilde{c}_{0}<t<1$, we see that $A_{k ; t} \cap \partial E=\emptyset$. Consequently, each connected component of the open set $A_{k ; t}$ lies either in the interior of $E$ or in its exterior, and thus must lie in its interior.

30.1. Corollary. Let $E$ be a bounded set of finite perimeter with uniform Lipschitz boundary. Then there exists $T \in(0,1)$ such that $A_{k ; T} \Subset E$.

Proof. Since $E$ has a uniform Lipschitz boundary, for each $x \in \partial E$, there is a finite cone, $C_{x}$, with vertex $x$ that completely lies in the complement of $E$. Each cone $C_{x}$ is assumed to be congruent to a fixed cone $C$. This implies that the hypothesis of Theorem 29.1 is satisfied. Therefore, there exists $0<T<1$ such that $u_{k}(y)<T$ for all $k$ and all $y \in \partial E$.

30.2. Definition. An open set $U \subset \mathbb{R}^{N}$ is called an extension domain for $\boldsymbol{F} \in \mathcal{D} \mathcal{M}^{\infty}(U)$, if there exists a field $\boldsymbol{F}^{*} \in \mathcal{D} \mathcal{M}^{\infty}\left(\mathbb{R}^{N}\right)$ such that $\boldsymbol{F}=\boldsymbol{F}^{*}$ on $U$.

30.3. Theorem. An open set $U$ satisfying $\mathcal{H}^{N-1}(\partial U)<\infty$ is an extension domain for any $\boldsymbol{F} \in$ $\mathcal{D M}^{\infty}(U)$. More generally, if $\boldsymbol{F} \in \mathcal{D} \mathcal{M}_{\text {loc }}^{\infty}(\Omega)$, then any open set of finite perimeter $U \Subset \Omega$ is an extension domain for $\boldsymbol{F}$.

Proof. We define an extension of $\boldsymbol{F}$ by

$$
\boldsymbol{F}^{*}(y):=\chi_{U}(y) \boldsymbol{F}(y) \text { for all } y \in \mathbb{R}^{N} .
$$

According to Definition 7.3 it suffices to show that

$$
\sup \left\{\int_{\mathbb{R}^{N}} \boldsymbol{F}^{*} \cdot \nabla \varphi:|\varphi| \leq 1, \varphi \in C_{c}^{\infty}\left(\mathbb{R}^{N}\right)\right\}<\infty .
$$

We consider first the case $\mathcal{H}^{N-1}(U)<\infty$. Let $U_{k}$ be the sequence of approximate sets given in Proposition 28.2. Therefore, for any $\varphi \in C_{c}^{\infty}\left(\mathbb{R}^{N}\right)$ with $|\varphi| \leq 1$, we employ our general Gauss-Green theorem, Theorem 21.1, to obtain

$$
\int_{U_{k}} \boldsymbol{F} \cdot \nabla \varphi d y+\int_{U_{k}} \varphi \operatorname{div} \boldsymbol{F}=-\int_{\partial U_{k}} \varphi \mathcal{F}_{i} \cdot \boldsymbol{\nu} d \mathcal{H}^{N-1}
$$

Thus,

$$
\begin{aligned}
\int_{U_{k}} \boldsymbol{F} \cdot \nabla \varphi d y & =-\int_{U_{k}} \varphi \operatorname{div} \boldsymbol{F}-\int_{\partial U_{k}} \varphi \mathcal{F}_{i} \cdot \boldsymbol{\nu} d \mathcal{H}^{N-1} \\
& \leq\|\operatorname{div} \boldsymbol{F}\|\left(U_{k}\right)+\|\boldsymbol{F}\|_{\infty} \mathcal{H}^{N-1}\left(\partial U_{k}\right) \\
& \leq\|\operatorname{div} \boldsymbol{F}\|(U)+\|\boldsymbol{F}\|_{\infty} \mathcal{H}^{N-1}\left(\partial U_{k}\right)
\end{aligned}
$$

Letting $k \rightarrow \infty$, we obtain

$$
\int_{U} \boldsymbol{F} \cdot \nabla \varphi d y \leq\|\operatorname{div} \boldsymbol{F}\|(U)+\|\boldsymbol{F}\|_{\infty} \mathcal{H}^{N-1}(\partial U)<\infty .
$$

Thus,

$$
\int_{U} \boldsymbol{F}^{*} \cdot \nabla \varphi d y=\int_{U} \boldsymbol{F} \cdot \nabla \varphi d y<\infty
$$


We now consider the case that $U \Subset \Omega$ is a set of finite perimeter and $\boldsymbol{F} \in \mathcal{D} \mathcal{M}_{\text {loc }}^{\infty}(\Omega)$. Proceeding as above and using Theorem 21.1.

$$
\begin{aligned}
\int_{\mathbb{R}^{N}} \boldsymbol{F}^{*} \cdot \nabla \varphi d y & =\int_{U} \boldsymbol{F}^{*} \cdot \nabla \varphi d y \\
& =-\int_{U} \varphi \operatorname{div} \boldsymbol{F}-\int_{\partial^{*} U} \varphi \mathcal{F}_{i} \cdot \boldsymbol{\nu} d \mathcal{H}^{N-1} \\
& \leq\|\operatorname{div} \boldsymbol{F}\|(U)+\|\boldsymbol{F}\|_{\infty} \mathcal{H}^{N-1}\left(\partial^{*} U\right)<\infty .
\end{aligned}
$$

31.1. Corollary (Chen-Torres [CT05]). Let $U \subset \mathbb{R}^{N}$ be a bounded, open set with $\mathcal{H}^{N-1}(\partial U)<\infty$. Let $\boldsymbol{F}_{1} \in \mathcal{D} \mathcal{M}^{\infty}(U)$ and $\boldsymbol{F}_{2} \in \mathcal{D} \mathcal{M}^{\infty}\left(\mathbb{R}^{N} \backslash \bar{U}\right)$. Then, with

we have

$$
\boldsymbol{F}(y):= \begin{cases}\boldsymbol{F}_{1}(y) & y \in U, \\ \boldsymbol{F}_{2}(y) & y \in \mathbb{R}^{N} \backslash \bar{U},\end{cases}
$$

$$
\boldsymbol{F} \in \mathcal{D} \mathcal{M}^{\infty}\left(\mathbb{R}^{N}\right)
$$

Proof. Applying the previous result to

$$
\boldsymbol{F}_{1}^{*}:=\chi_{U}(y) \boldsymbol{F}_{1}(y) \text { for all } y \in \mathbb{R}^{N}
$$

and

we see that

$$
\boldsymbol{F}_{2}^{*}:=\chi_{\mathbb{R}^{N} \backslash \bar{U}} \boldsymbol{F}_{2}(y) \text { for all } y \in \mathbb{R}^{N},
$$

$$
\boldsymbol{F}=\boldsymbol{F}_{1}^{*}+\boldsymbol{F}_{2}^{*}
$$

\section{Cauchy Fluxes and Divergence-Measure Fields}

The physical principle of balance law of the form

$$
\int_{\partial E} f(y, \boldsymbol{\nu}(y)) d \mathcal{H}^{N-1}(y)+\int_{E} b(y) d y=0
$$

is basic in all of classical physics. Here, $\boldsymbol{\nu}(y)$ is the interior unit normal to the boundary $\partial E$ of $E$. In mechanics, $f$ represents the surface force per unit area on $\partial E$, while in thermodynamics $f$ gives the heat flow per unit area across the boundary $\partial E$.

In 1823, Cauchy [Ca1823. (also see Ca1827]) established the stress theorem that is probably the most important result in continuum mechanics: If both $f(y, \boldsymbol{\nu}(y))$, defined for each $y$ in an open region $\Omega$ and every unit vector $\boldsymbol{\nu}$, is continuous in $y$ and $b(y)$ is uniformly bounded on $\Omega$, and if (31.1) is satisfied for every smooth region $E \Subset \Omega$, then $f(y, \boldsymbol{\nu})$ must be linear in $\boldsymbol{\nu}$. The Cauchy postulate states that the density flux $f$ through a surface depends on the surface solely through the normal at that point. For instance, if $f(y, \boldsymbol{\nu})$ represents the heat flow, then the Stress theorem states that there exists a vector field $\boldsymbol{F}$ such that

$$
f(y, \boldsymbol{\nu})=\boldsymbol{F}(y) \cdot \boldsymbol{\nu} .
$$

Since the time of Cauchy's stress result, Ca1823, Ca1827, many efforts have been made to generalize his ideas and remove some of his hypotheses. The first results in this direction were obtained by Noll Nol59 in 1959, who set up a basis for an axiomatic foundation for continuum thermodynamics. In particular, Noll [Nol59] showed that the Cauchy postulate may directly follow from the balance law. In GM76, Gurtin-Martins introduced the concept of Cauchy flux and removed the continuity assumption on $f$. In [Zie83, Ziemer proved Noll's theorem in the context of geometric measure theory, in which the Cauchy fluxes were first formulated at the level of generality with sets of finite perimeter in the absence of jump surfaces, "shock waves". 
However, as we explain below, all the previous formulations of (31.1) do not allow the presence of "shock waves"; one of our main intentions in this paper is to develop a theory that will allow the presence of "shock waves".

In this section we first introduce a class of Cauchy fluxes that allows the presence of the exceptional surfaces or "shock waves" and then prove that such a Cauchy flux induces a bounded divergencemeasure (vector) field $\boldsymbol{F}$ so that the Cauchy flux over every oriented surface can be recovered through $\boldsymbol{F}$ and the normal to the oriented surface. Before introducing this framework, we need the following definitions.

32.1. Definition. An oriented surface in $\Omega$ is a pair $(S, \boldsymbol{\nu})$ so that $S \Subset \Omega$ is a Borel set and $\nu: \mathbb{R}^{N} \rightarrow \mathbb{S}^{N-1}$ is a Borel measurable unit vector field that satisfy the following property: There is a set $E \Subset \Omega$ of finite perimeter such that $S \subset \partial^{*} E$ and

$$
\boldsymbol{\nu}(y)=\boldsymbol{\nu}_{E}(y) \chi_{S}(y)
$$

where $\chi_{S}$ is the characteristic function of the set $S$ and $\boldsymbol{\nu}_{E}(y)$ is the interior measure-theoretic unit normal to $E$ at $y$.

Two oriented surfaces $\left(S_{j}, \boldsymbol{\nu}_{j}\right), j=1,2$, are said to be compatible if there exists a set of finite perimeter $E$ such that $S_{j} \subset \partial^{*} E$ and $\boldsymbol{\nu}_{j}(y)=\boldsymbol{\nu}_{E}(y) \chi_{S_{j}}(y), j=1,2$. For simplicity, we will denote the pair $(S, \boldsymbol{\nu})$ simply as $S$, with implicit understanding that $S$ is oriented by the interior normal of some set $E$ of finite perimeter. We define $-S=(S,-\boldsymbol{\nu})$, which is regarded as a different oriented surface.

32.2. Definition. Let $\Omega$ be a bounded open set. A Cauchy flux is a functional $\mathcal{F}$ that assigns to each oriented surface $S:=(S, \boldsymbol{\nu}) \Subset \Omega$ a real number and has the following properties:

(i) $\mathcal{F}\left(S_{1} \cup S_{2}\right)=\mathcal{F}\left(S_{1}\right)+\mathcal{F}\left(S_{2}\right)$ for any pair of compatible disjoint surfaces $S_{1}, S_{2} \Subset \Omega$;

(ii) There exists a nonnegative Radon measure $\sigma$ in $\Omega$ such that

$$
\left|\mathcal{F}\left(\partial^{*} E\right)\right| \leq \sigma(E)
$$

for every set of finite perimeter $E \Subset \Omega$ satisfying $\sigma(\partial E)=0$;

(iii) There exists a constant $C$ such that

$$
|\mathcal{F}(S)| \leq C \mathcal{H}^{N-1}(S)
$$

for every oriented surface $S \Subset \Omega$ satisfying $\sigma(S)=0$.

This general framework for Cauchy fluxes allows the presence of exceptional surfaces, "shock waves", in the formulation of the axioms, on which the measure $\sigma$ has support. On these exceptional surfaces, the Cauchy flux $\mathcal{F}$ has a discontinuity, i.e., $\mathcal{F}(S) \neq-\mathcal{F}(-S)$. In fact, the exceptional surfaces are supported on the singular part of measure $\sigma$. When $\sigma$ reduces to the $N$-dimensional Lebesgue measure $\mathcal{L}^{N}$, the formulation reduces to Ziemer's formulation in [Zie83] and in this case $\sigma$ vanishes on any $\mathcal{H}^{N-1}$-dimensional surface, which excludes shock waves.

The theory developed in this paper allows to approximate the exceptional surfaces or "shock waves" with smooth boundaries and rigorously pass to the limit to recover the flux across the shock waves. This allows to capture measure production density in the formulation of the balance law and entropy dissipation for entropy solutions of hyperbolic conservation laws. Once we know the flux across every surface, we proceed to obtain a rigorous derivation of nonlinear systems of balance laws with measure source terms from the physical principle of balance law in $\S 10$. The framework also allows the recovery of Cauchy entropy fluxes through the Lax entropy inequality for entropy solutions of hyperbolic conservation laws by capturing entropy dissipation; see $\S 11$.

The main theorem of this section is the following.

32.3. Theorem. Let $\mathcal{F}$ be a Cauchy flux in $\Omega$. Then there exists a unique divergence-measure field $\boldsymbol{F} \in \mathcal{D M}_{\text {loc }}^{\infty}(\Omega)$ such that

$$
\mathcal{F}(S)=-\int_{S} \mathcal{F}_{i} \cdot \boldsymbol{\nu} d \mathcal{H}^{N-1}
$$


for every oriented surface $(S, \boldsymbol{\nu}) \Subset \Omega$, where $\mathcal{F}_{i} \cdot \boldsymbol{\nu}$ is the normal trace of $\boldsymbol{F}$ to the oriented surface.

When $\sigma$ reduces to the $N$-dimensional Lebesgue measure $\mathcal{L}^{N}$, as in Ziemer's formulation, the vector field $\boldsymbol{F}$ satisfies $\operatorname{div} \boldsymbol{F} \in L^{\infty}$ and $\mathcal{F}(S)=-\mathcal{F}(-S)$ for every surface $S$, which thus excludes shock waves where the Cauchy flux $\mathcal{F}$ has a discontinuity, i.e., $\mathcal{F}(S) \neq-\mathcal{F}(-S)$.

In order to establish Theorem 32.3 we need Lemmas 33.2 35.1 that were first shown in DegiovanniMarzocci-Musesti [DMM99]. Here we offer simplified proofs of these facts for completeness. In particular, Lemma 33.2 is in fact a direct application of Theorem 33.1 (due to Fuglede) below, and Lemma 35.1 follows by an approximation and Theorem 21.1. We also refer to Schuricht [Sch07] for a different approach in formulating the axioms in Definition 32.2 ,

The following theorem, due to Fuglede, is a generalization of Riesz's theorem, whose proof can be found in Fug55.

33.1. Theorem. Let $\mu$ be a nonnegative measure defined on a $\sigma$-field $\mathcal{V}$ of subsets of a fixed set $X$ and $X \in \mathcal{V}$. Let $\varphi$ be an additive set function defined on a system of sets $\mathcal{U} \subset \mathcal{V}$ such that all finite unions of disjoint sets from $\mathcal{U}$, together with the empty set, form a field $\mathcal{F}$ which generates $\mathcal{V}$. Assume that $\mu(A)<\infty$ for every $A \subset \mathcal{U}$. Then there exists a function $g(y) \in L^{1}(X, \mathcal{V}, \mu)$ with the property that

if and only if the following hold:

$$
\varphi(A)=\int_{A} g(y) d \mu \quad \text { for every } A \in \mathcal{U}
$$

(i) For every $\varepsilon>0$, there exists $\delta>0$ such that $\sum_{i=1}^{n}\left|\varphi\left(A_{i}\right)\right| \leq \varepsilon$ for every finite system of disjoint sets $A_{1}, A_{2}, \ldots, A_{n}$ from $\mathcal{U}$ for which $\sum_{i=1}^{n} \mu\left(A_{i}\right)<\delta$;

(ii) There is a finite constant $C$ such that $\sum_{i=1}^{n}\left|\varphi\left(A_{i}\right)\right| \leq C$ for every finite system of disjoint sets $A_{1}, A_{2}, \ldots, A_{n}$ from $\mathcal{U}$.

The function $g$ is then essentially uniquely determined. Under the additional assumption that $\mu(X)<$ $\infty$, condition (ii) is a consequence of condition (i).

Let $\{I\}$ be the collection of all closed cubes in $\mathbb{R}^{N}$ of the form

$$
I=\left[a_{1}, b_{1}\right] \times\left[a_{2}, b_{2}\right] \times \ldots\left[a_{N}, b_{N}\right],
$$

where $a_{1}, b_{1}, a_{2}, b_{2}, \ldots, a_{N}$, and $b_{N}$ are real numbers. For almost every $\tau_{j} \in\left[a_{j}, b_{j}\right]$, we define

$$
I_{\tau_{j}}=\left\{y \in I: y_{j}=\tau_{j}\right\} .
$$

We define the vectors $e_{1}, e_{2}, \ldots, e_{N}$ so that the $j$-th component of $e_{j}$ is -1 and the other components are zero. We orient the surface $I_{\tau_{j}}$ with the vector $e_{j}$.

33.2. Lemma. Let $\mathcal{F}$ be a Cauchy flux in $\Omega$. Then there exists a divergence-measure field $\boldsymbol{F} \in$ $\mathcal{D M}_{\text {loc }}^{\infty}(\Omega)$ such that, for every cube $I=\left[a_{1}, b_{1}\right] \times\left[a_{2}, b_{2}\right] \times \ldots \times\left[a_{N}, b_{N}\right] \Subset \Omega$ and almost every $\tau_{j} \in\left[a_{j}, b_{j}\right]$,

$$
\mathcal{F}\left(I_{\tau_{j}}\right)=-\int_{I_{\tau_{j}}} \boldsymbol{F}(y) \cdot e_{j} d \mathcal{H}^{N-1}(y) .
$$

Proof. Step 1. We fix $j \in\{1, \ldots, N\}$. For every cube $I \subset \Omega$, we define

$$
\mu^{j}(I)=\int_{a_{j}}^{b_{j}} \mathcal{F}\left(I_{\tau_{j}}\right) d \tau_{j}
$$

We have

$$
\left|\mu^{j}(I)\right| \leq \int_{a_{j}}^{b_{j}}\left|\mathcal{F}\left(I_{\tau_{j}}\right)\right| d \tau_{j} \leq C \int_{a_{j}}^{b_{j}} \int_{I_{\tau_{j}}} d \mathcal{H}^{N-1} d \tau_{j}=C|I| .
$$

Thus, from Theorem 33.1 there exists a function $f^{j} \in L^{1}(\Omega)$ such that

$$
\mu^{j}(I)=\int_{I} f^{j} d y \quad \text { for every } I .
$$




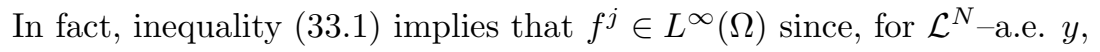

$$
f^{j}(y)=\lim _{|I| \rightarrow 0, y \in I} \frac{\int_{I} f^{j} d x}{|I|} \leq C .
$$

Fubini's theorem implies that

$$
\mu^{j}(I)=\int_{a_{j}}^{b_{j}} \mathcal{F}\left(I_{\tau_{j}}\right) d \tau_{j}=\int_{I} f^{j} d y=\int_{a_{j}}^{b_{j}} \int_{I_{\tau_{j}}} f^{j} d \mathcal{H}^{N-1} d \tau_{j} .
$$

Let $\tau_{j} \in\left[a_{j}, b_{j}\right], \alpha_{k, j}$, and $\beta_{k, j}$ be sequences such that

$$
\alpha_{k, j} \leq \tau_{j} \leq \beta_{k, j},
$$

where $\alpha_{k, j}$ is an increasing sequence that converges to $\tau_{j}$ as $k \rightarrow \infty$, and $\beta_{k, j}$ is a decreasing sequence that converges to $\tau_{j}$ as $k \rightarrow \infty$. Thus, from (34.1), we obtain

$$
\frac{1}{\alpha_{k, j}-\beta_{k, j}} \int_{a_{j}}^{b_{j}} \mathcal{F}\left(I_{\tau_{j}}\right) d \tau_{j}=\frac{1}{\alpha_{k, j}-\beta_{k, j}} \int_{a_{j}}^{b_{j}} \int_{I_{\tau_{j}}} f^{j} d \mathcal{H}^{N-1} d \tau_{j} .
$$

We let $k \rightarrow \infty$ to obtain that, for a.e. $\tau_{j}$,

$$
\mathcal{F}\left(I_{\tau_{j}}\right)=\int_{I_{\tau_{j}}} f^{j} d \mathcal{H}^{N-1}
$$

Define

$$
\boldsymbol{F}:=\left(f^{1}, f^{2}, \ldots, f^{N}\right) .
$$

Then we find that, for almost every $\tau_{j}, j \in\{1,2, \ldots, N\}$,

$$
\mathcal{F}\left(I_{\tau_{j}}\right)=-\int_{I_{\tau_{j}}} \boldsymbol{F}(y) \cdot e_{j} d \mathcal{H}^{N-1}(y) .
$$

Step 2. We now prove that the divergence of $\boldsymbol{F}$, in the sense of distributions, is a measure. We define, for a.e. cube $I$,

$$
\eta(I):=-\int_{\partial I} \boldsymbol{F}(y) \cdot \boldsymbol{\nu}(y) d \mathcal{H}^{N-1}(y),
$$

where $\boldsymbol{\nu}$ is the interior unit normal to $\partial I$.

From Step 1 and the definition of Cauchy flux, we have

$$
|\eta(I)|=|\mathcal{F}(\partial I)| \leq \sigma(I)
$$

for almost all closed cubes. Thus, we can again apply Theorem 33.1 to conclude that there exists a function $g \in L^{1}(\Omega ; \sigma)$, uniquely defined in $\Omega$ up to a set of $\sigma$-measure zero, such that

$$
\eta(I)=-\int_{\partial I} \boldsymbol{F}(y) \cdot \boldsymbol{\nu}(y) d \mathcal{H}^{N-1}(y)=\int_{I} g(y) d \sigma
$$

for almost every closed cube $I \subset \Omega$.

Denote $\tilde{\sigma}$ the measure given by $g d \sigma$ in $\Omega$. We now prove

$$
\operatorname{div} \boldsymbol{F}=\tilde{\sigma}
$$

in the sense of distributions in any open set $U \Subset \Omega$.

Let $I \Subset U$ be any closed cube. Then, for any $\phi \in C^{1}$ with support contained in $I$,

$$
\int_{U} \boldsymbol{F} \cdot \nabla \phi d y=\lim _{\varepsilon \rightarrow 0} \int_{U} \boldsymbol{F}_{\varepsilon} \cdot \nabla \phi d y=-\lim _{\varepsilon \rightarrow 0} \int_{U} \phi \operatorname{div} \boldsymbol{F}_{\varepsilon} d y,
$$

where $\boldsymbol{F}_{\varepsilon}=\boldsymbol{F} * \rho_{\varepsilon}$ and $\rho$ is the standard mollifying kernel. We now prove that, for $\mathcal{L}^{N}$-a.e. $y \in U$,

$$
\tilde{\sigma}_{\varepsilon}(y)=\operatorname{div} \boldsymbol{F}_{\varepsilon}(y),
$$


where $\tilde{\sigma}_{\varepsilon}$ is the convolution of function $\rho_{\varepsilon}$ with the measure $\tilde{\sigma}$; that is,

$$
\tilde{\sigma}_{\varepsilon}(y):=\left(\rho_{\varepsilon} * \tilde{\sigma}\right)(y)=\int_{\Omega} \rho_{\varepsilon}(y-x) d \tilde{\sigma}(x) .
$$

From (34.6)-(34.7), we find that, for $\varepsilon<\operatorname{dist}(\partial U, \partial \Omega)$,

$$
\begin{aligned}
\int_{I} \operatorname{div} \boldsymbol{F}_{\varepsilon}(y) d y & =-\int_{\partial I} \boldsymbol{F}_{\varepsilon}(y) \cdot \boldsymbol{\nu}(y) d y=-\int_{\partial I} \int_{\mathbb{R}^{N}} \boldsymbol{F}(y-x) \cdot \boldsymbol{\nu}(y) \rho_{\varepsilon}(x) d x d y \\
& =-\int_{\mathbb{R}^{N}} \int_{\partial I} \boldsymbol{F}(y-x) \cdot \boldsymbol{\nu}(y) \rho_{\varepsilon}(x) d y d x \\
& =-\int_{\mathbb{R}^{N}}\left(\int_{\partial I_{x}} \boldsymbol{F}(y) \cdot \boldsymbol{\nu}(y) d y\right) \rho_{\varepsilon}(x) d x \\
& =\int_{\mathbb{R}^{N}} \tilde{\sigma}\left(I_{x}\right) \rho_{\varepsilon}(x) d x
\end{aligned}
$$

where $I_{x}=\left\{y: a_{i} \leq y_{i}-x_{i} \leq b_{i}, i=1, \cdots, N\right\}$. We can consider the smooth function $\rho_{\varepsilon}$ as a measure in $\mathbb{R}^{N}$, say $\lambda_{\varepsilon}$, by defining $\lambda_{\varepsilon}(A)=\int_{A} \rho_{\varepsilon}(x) d x$ for any Borel set $A$. We can also extend the measure $\tilde{\sigma}$ by zero outside $\Omega$. Therefore, we find

$$
\int_{\mathbb{R}^{N}} \tilde{\sigma}\left(I_{x}\right) \rho_{\varepsilon}(x) d x=\left(\tilde{\sigma} * \lambda_{\varepsilon}\right)(I)=\left(\lambda_{\varepsilon} * \tilde{\sigma}\right)(I)=\int_{\mathbb{R}^{N}} \lambda_{\varepsilon}\left(I_{x}\right) d \tilde{\sigma}(x) .
$$

From (35.1) and using (34.10), we compute

$$
\begin{aligned}
\int_{\mathbb{R}^{N}} \lambda_{\varepsilon}\left(I_{x}\right) d \tilde{\sigma}(x)=\int_{\Omega} \lambda_{\varepsilon}\left(I_{x}\right) d \tilde{\sigma}(x) & =\int_{\Omega}\left(\int_{I_{x}} \rho_{\varepsilon}(y) d y\right) d \tilde{\sigma}(x)=\int_{\Omega} \int_{I} \rho_{\varepsilon}(y-x) d y d \tilde{\sigma}(x) \\
& =\int_{I}\left(\int_{\Omega} \rho_{\varepsilon}(y-x) d \tilde{\sigma}(x)\right) d y=\int_{I}\left(\rho_{\varepsilon} * \tilde{\sigma}\right)(y) d y \\
& =\int_{I} \tilde{\sigma}_{\varepsilon}(y) d y .
\end{aligned}
$$

Therefore,

$$
\int_{I} \operatorname{div} \boldsymbol{F}_{\varepsilon}(y) d y=\int_{I} \tilde{\sigma}_{\varepsilon}(y) d y .
$$

Since the cube $I \Subset U$ is arbitrary, this shows that $\tilde{\sigma}_{\varepsilon}(y)=\operatorname{div} \boldsymbol{F}_{\varepsilon}(y)$ for $\mathcal{L}^{N}$-a.e. $y \in U$. Using this in (34.9), we obtain

$$
\int_{U} \boldsymbol{F} \cdot \nabla \phi d y=-\lim _{\varepsilon \rightarrow 0} \int_{U} \phi \operatorname{div} \boldsymbol{F}_{\varepsilon} d y=-\lim _{\varepsilon \rightarrow 0} \int_{U} \phi \tilde{\sigma}_{\varepsilon} d y=-\int_{U} \phi(y) d \tilde{\sigma}(y),
$$

since the sequence of measures $\tilde{\sigma}_{\varepsilon}$ converges locally weak* in $\Omega$ to $\tilde{\sigma}$ as $\varepsilon \rightarrow 0$.

35.1. Lemma. Let $\mathcal{F}$ be a Cauchy flux in $\Omega$. Then there exists a unique divergence-measure field $\boldsymbol{F} \in \mathcal{D M}_{\text {loc }}^{\infty}(\Omega)$ such that

$$
\mathcal{F}(S)=-\int_{S} \mathcal{F}_{i} \cdot \boldsymbol{\nu} d \mathcal{H}^{N-1}
$$

for almost every oriented surface $(S, \boldsymbol{\nu}) \Subset \Omega$; that is, every surface $S$ in $\Omega$ satisfying the condition that $\sigma(S)=0$.

Proof. Using Lemma 33.2 it follows that there exists an $\boldsymbol{F} \in \mathcal{D} \mathcal{M}_{\text {loc }}^{\infty}(\Omega)$ such that, for any cube $I=\left[a_{1}, b_{1}\right] \times\left[a_{2}, b_{2}\right] \times \ldots \times\left[a_{N}, b_{N}\right] \Subset \Omega$,

$$
\mathcal{F}\left(I_{\tau_{j}}\right)=-\int_{I_{\tau_{j}}} \boldsymbol{F}(y) \cdot e_{j} d \mathcal{H}^{N-1}(y)
$$

for almost every $\tau_{j} \in\left[a_{j}, b_{j}\right]$. 
Let $(S, \boldsymbol{\nu})$ be an oriented surface satisfying $\sigma(S)=0$. Then, since $\operatorname{div} \boldsymbol{F}=g d \sigma$ and proceeding as in Lemma 26.3, we conclude that $\|\operatorname{div} \boldsymbol{F}\|(S)=0$. We approximate $S$ with closed cubes such that

$$
S=\bigcap_{i=1}^{\infty} J_{i}
$$

where each $J_{i}$ is a finite union of closed cubes and $J_{i+1} \subset J_{i}$. Since $S$ is an oriented surface, there exists a set of finite perimeter, $E$, such that $S \subset \partial^{*} E$. Using Lemma 6.2, we have

$$
\partial^{*}\left(J_{i} \cap E\right)=S \cup\left(\partial^{*} J_{i} \cap E\right) \cup \mathcal{N}_{i},
$$

where $\lim _{i \rightarrow \infty} \mathcal{H}^{N-1}\left(\mathcal{N}_{i}\right)=0$ and thus, since $\mathcal{F}\left(\mathcal{N}_{i}\right) \leq C \mathcal{H}^{N-1}\left(\mathcal{N}_{i}\right)$, we obtain

$$
\lim _{i \rightarrow \infty} \mathcal{F}\left(\mathcal{N}_{i}\right)=0 .
$$

The definition of Cauchy flux implies that

$$
\left|\mathcal{F}\left(\partial^{*}\left(J_{i} \cap E\right)\right)\right| \leq \sigma\left(J_{i} \cap E\right),
$$

and thus (35.5) implies that

$$
\lim _{i \rightarrow \infty}\left|\mathcal{F}\left(\partial^{*}\left(J_{i} \cap E\right)\right)\right| \leq \sigma(S)=0 .
$$

On the other hand, using Theorem 21.1, we have

$$
\left|-\int_{\partial^{*}\left(J_{i} \cap E\right)} \mathcal{F}_{i} \cdot \boldsymbol{\nu} d \mathcal{H}^{N-1}\right|=\left|\int_{J_{i} \cap E} \operatorname{div} \boldsymbol{F}\right| \leq\|\operatorname{div} \boldsymbol{F}\|\left(J_{i} \cap E\right),
$$

which yields

$$
\lim _{i \rightarrow \infty}\left|-\int_{\partial^{*}\left(J_{i} \cap E\right)} \mathcal{F}_{i} \cdot \boldsymbol{\nu} d \mathcal{H}^{N-1}\right| \leq\|\operatorname{div} \boldsymbol{F}\|(S)=0 .
$$

Using (36.1)-(36.3) and Lemma 33.2, we obtain

$$
\begin{aligned}
\lim _{i \rightarrow \infty}\left|\mathcal{F}\left(\partial^{*}\left(J_{i} \cap E\right)\right)\right| & =\lim _{i \rightarrow \infty}\left|\mathcal{F}(S)+\mathcal{F}\left(\partial^{*} J_{i} \cap E\right)\right| \\
& =\lim _{i \rightarrow \infty}\left|\mathcal{F}(S)-\int_{\partial^{*} J_{i} \cap E} \boldsymbol{F}(y) \cdot \boldsymbol{\nu}(y) d \mathcal{H}^{N-1}\right|=0 .
\end{aligned}
$$

From (36.1), (36.2), and (36.5), we obtain

$$
\lim _{i \rightarrow \infty}\left|-\int_{\partial^{*} J_{i} \cap E} \boldsymbol{F}(y) \cdot \boldsymbol{\nu}(y) d \mathcal{H}^{N-1}-\int_{S} \mathcal{F}_{i} \cdot \boldsymbol{\nu} d \mathcal{H}^{N-1}\right|=0 .
$$

Combining (36.6) with (36.7) yields

$$
\mathcal{F}(S)=-\int_{S} \mathcal{F}_{i} \cdot \boldsymbol{\nu} d \mathcal{H}^{N-1}
$$

Assume now that there exists another vector field $G=\left(g^{1}, g^{2}, \ldots, g^{N}\right)$ such that (35.3) holds. Then, for fixed $j \in\{1,2, \ldots, N\}$, we have

$$
\int_{I} f^{j} d y=\int_{a_{j}}^{b_{j}} \int_{I_{\tau_{j}}} f^{j} d \mathcal{H}^{N-1} d \tau_{j}=\int_{a_{j}}^{b_{j}} \int_{I_{\tau_{j}}} g^{j} d \mathcal{H}^{N-1} d \tau_{j}=\int_{I} g^{j} d y
$$

for any cube $I$. This implies that

$$
f^{j}(y)=g^{j}(y) \quad \text { for almost every } y .
$$

With Lemmas 33.2 35.1, we now prove Theorem 32.3 to explain how the Cauchy flux can be recovered on the exceptional surfaces based on the theory established in $\S 3-\S 8$. 
Proof of Theorem 32.3. Let $(S, \boldsymbol{\nu})$ be an oriented surface on which $\sigma(S) \neq 0$, i.e. $\mathcal{F}(-S) \neq-\mathcal{F}(S)$. By definition of oriented surfaces, there exists a bounded set of finite perimeter, $E:=E^{1} \cup \partial^{*} E \Subset \Omega$, such that

$$
S \subset \partial^{*} E \quad \text { and } \quad \boldsymbol{\nu}(y)=\boldsymbol{\nu}_{E}(y) \chi_{S}(y),
$$

where $\boldsymbol{\nu}_{E}(y)$ is the interior normal to $E$ at $y \in S$. Consider

$$
\tilde{E}=E^{0} \cup \partial^{*} E .
$$

Then Theorem 21.1implies that there exist the normal traces $\mathcal{F}_{i} \cdot \boldsymbol{\nu}$ and $\mathcal{F}_{e} \cdot \boldsymbol{\nu}$ defined on $\partial^{*} \tilde{E}=\partial^{*} E$ respectively such that

$$
\begin{aligned}
\int_{E^{1}} \operatorname{div} \boldsymbol{F} & =-\int_{\partial^{*} E} \mathcal{F}_{i} \cdot \boldsymbol{\nu} d \mathcal{H}^{N-1}, \\
\int_{E} \operatorname{div} \boldsymbol{F} & =-\int_{\partial^{*} \tilde{E}} \mathcal{F}_{e} \cdot \boldsymbol{\nu} d \mathcal{H}^{N-1}=-\int_{\partial^{*} E} \mathcal{F}_{e} \cdot \boldsymbol{\nu} d \mathcal{H}^{N-1} .
\end{aligned}
$$

Theorem 21.1 indicates that the traces $\mathcal{F}_{i} \cdot \boldsymbol{\nu}$ and $\mathcal{F}_{e} \cdot \boldsymbol{\nu}$ can be recovered, up to a set of arbitrary small $\mathcal{H}^{N-1}$-measure, from the neighborhood behavior of the vector field $\boldsymbol{F}$. This observation allows us to define

$$
\mathcal{F}(S)=\mathcal{F}(S, \boldsymbol{\nu}):=-\int_{S} \mathcal{F}_{i} \cdot \boldsymbol{\nu} d \mathcal{H}^{N-1}
$$

and

$$
\mathcal{F}(-S)=\mathcal{F}(S,-\boldsymbol{\nu}):=-\int_{S} \mathcal{F}_{e} \cdot(-\boldsymbol{\nu}) d \mathcal{H}^{N-1}=\int_{S} \mathcal{F}_{e} \cdot \boldsymbol{\nu} d \mathcal{H}^{N-1} .
$$

In this way, we can recover the Cauchy flux $\mathcal{F}$ through the corresponding divergence-measure field $\boldsymbol{F}$ over all oriented surfaces, especially including the exceptional surfaces. That is, the normal traces of $\boldsymbol{F} \in \mathcal{D M}_{l o c}^{\infty}(\Omega)$ are the Cauchy densities over all oriented surfaces. This completes the proof of Theorem 32.3 .

\section{Mathematical formulation of the balance law and Derivation of Systems of BALANCE LAWS}

In this section we first present the mathematical formulation of the physical principle of balance law (31.1). Then we apply the results established in $\S 3-\S 9$ to give a rigorous derivation of systems of balance laws with measure source terms. In particular, we give a derivation of hyperbolic systems of conservation laws (38.7).

A balance law on an open subset $\Omega$ of $\mathbb{R}^{N}$ postulates that the production of a vector-valued "extensive" quantity in any bounded measurable subset $E \Subset \Omega$ with finite perimeter is balanced by the Cauchy flux of this quantity through the measure-theoretic boundary $\partial^{m} E$ of $E$ (see Dafermos Daf98, Daf05).

Like the Cauchy flux, the production is introduced through a functional $\mathcal{P}$, defined on any bounded measurable subset of finite perimeter, $E \subset \Omega$, taking value in $\mathbb{R}^{k}$ and satisfying the conditions:

$$
\begin{aligned}
& \mathcal{P}\left(E_{1} \cup E_{2}\right)=\mathcal{P}\left(E_{1}\right)+\mathcal{P}\left(E_{2}\right) \quad \text { if } E_{1} \cap E_{2}=\emptyset, \\
& |\mathcal{P}(E)| \leq \sigma(E) .
\end{aligned}
$$

Then the physical principle of balance law can be mathematically formulated as

$$
\mathcal{F}\left(\partial^{m} E\right)=\mathcal{P}(E)
$$

for any bounded measurable subset of finite perimeter, $E \subset \Omega$. 
Fugele's theorem, Theorem 33.1, indicates that conditions (37.4)-(37.5) implies that there is a production density $P \in \mathcal{M}\left(\Omega ; \mathbb{R}^{k}\right)$ such that

$$
\mathcal{P}(E)=\int_{E^{1}} P(y) .
$$

On the other hand, combining Theorem 21.1 with the argument from $\S 9$, it follows that there exists $\boldsymbol{F} \in \mathcal{D} \mathcal{M}_{\text {loc }}^{\infty}\left(\Omega ; \mathbb{R}^{N \times k}\right)$ such that

$$
\mathcal{F}\left(\partial^{m} E\right)=-\int_{\partial^{m} E}\left(\mathcal{F}_{i} \cdot \boldsymbol{\nu}\right) d \mathcal{H}^{N-1}=\int_{E^{1}} \operatorname{div} \boldsymbol{F}(y)
$$

for any set of finite perimeter, $E \Subset \Omega$.

Then (37.6)-38.1 yields the following system of field equations

$$
\operatorname{div} \boldsymbol{F}(y)=P(y)
$$

in the sense of measures on $\Omega$.

We assume that the state of the medium is described by a state vector field $u$, taking value in an open subset $U$ of $\mathbb{R}^{k}$, which determines both the flux density field $\boldsymbol{F}$ and the production density field $P$ at the point $y \in \Omega$ by the constitutive equations:

$$
\boldsymbol{F}(y):=\boldsymbol{F}(u(y), y), \quad P(y):=P(u(y), y),
$$

where $\boldsymbol{F}(u, y)$ and $P(u, y)$ are given smooth functions defined on $U \times \Omega$.

Combining (38.2) with (38.3) leads to the quasilinear first-order system of partial differential equations

$$
\operatorname{div} \boldsymbol{F}(u(y), y)=P(u(y), y),
$$

which is called a system of balance laws (cf. Daf98).

If $\mathcal{P}=0$, the previous derivation yields

$$
\operatorname{div} \boldsymbol{F}(u(y), y)=0,
$$

which is called a system of conservation laws. When the medium is homogeneous:

$$
\boldsymbol{F}(u, y)=\boldsymbol{F}(u)
$$

that is, $\boldsymbol{F}$ depends on $y$ only through the state vector, then system (38.5) becomes

$$
\operatorname{div} \boldsymbol{F}(u(y))=0 .
$$

In particular, when the coordinate system $y$ is described by the time variable $t$ and the space variable $x=\left(x_{1}, \cdots, x_{n}\right)$ :

$$
y=\left(t, x_{1}, \cdots, x_{n}\right)=(t, x), \quad N=n+1,
$$

and the flux density is written as

$$
\boldsymbol{F}(u)=\left(u, f_{1}(u), \cdots, f_{n}(u)\right)=(u, f(u)),
$$

then we have the following standard form for the system of conservation laws:

$$
\partial_{t} u+\nabla_{x} \cdot f(u)=0, \quad x \in \mathbb{R}^{n}, u \in \mathbb{R}^{k} .
$$




\section{Entropy Solutions of Hyperbolic Conservation Laws}

We now apply the results established in $\S 3-\S 9$ to the recovery of Cauchy entropy fluxes through the Lax entropy inequality for entropy solutions of hyperbolic conservation laws by capturing entropy dissipation. We focus on system (38.7) which is assumed to be hyperbolic.

38.1. Definition. A function $\eta: \mathbb{R}^{k} \rightarrow \mathbb{R}$ is called an entropy of (38.7) if there exists $q: \mathbb{R}^{k} \rightarrow \mathbb{R}^{n}$ such that

$$
\nabla q_{j}(u)=\nabla \eta(u) \nabla f_{j}(u), \quad j=1,2, \ldots, n .
$$

Then the vector function $q(u)$ is called an entropy flux associated with the entropy $\eta(u)$, and the pair $(\eta(u), q(u))$ is called an entropy pair. The entropy pair $(\eta(u), q(u))$ is called a convex entropy pair on the domain $U \subset \mathbb{R}^{k}$ if the Hessian matrix $\nabla^{2} \eta(u) \geq 0$ for any $u \in U$. The entropy pair $(\eta(u), q(u))$ is called a strictly convex entropy pair on the domain $U$ if $\nabla^{2} \eta(u)>0$ for any $u \in U$.

Friedrichs-Lax [FL71] observed that most of systems of conservation laws that result from continuum mechanics are endowed with a globally defined, strictly convex entropy. The available existence theories show that solutions of (38.7) are generally in the following class of entropy solutions.

39.1. Definition. A vector function $u=u(t, x) \in L_{l o c}^{\infty}\left(\mathbb{R}_{+} \times \mathbb{R}^{n}\right)$ is called an entropy solution if $u(t, x)$ satisfies the Lax entropy inequality:

$$
\partial_{t} \eta(u(t, x))+\nabla_{x} \cdot q(u(t, x)) \leq 0
$$

in the sense of distributions for any convex entropy pair $(\eta, q): \mathbb{R}^{k} \rightarrow \mathbb{R} \times \mathbb{R}^{n}$.

Clearly, an entropy solution is a weak solution by choosing $\eta(u)= \pm u$ in (39.1).

One of the main issues in conservation laws is to study the behavior of entropy solutions in this class to explore to the fullest extent possible all questions relating to large-time behavior, uniqueness, stability, structure, and traces of entropy solutions, with neither specific reference to any particular method for constructing the solutions nor additional regularity assumptions. The Schwartz lemma infers from (39.1) that the distribution

$$
\partial_{t} \eta(u(t, x))+\nabla_{x} \cdot q(u(t, x))
$$

is in fact a Radon measure, that is, the field $(\eta(u(t, x)), q(u(t, x)))$ is a divergence-measure field. Then there exists $\mu_{\eta} \in \mathcal{M}\left(\mathbb{R}_{+} \times \mathbb{R}^{n}\right)$ with $\mu_{\eta} \leq 0$ such that

$$
\operatorname{div}_{(t, x)}(\eta(u(t, x)), q(u(t, x)))=\mu_{\eta} .
$$

For any $L^{\infty}$ entropy solution $u$, it is first indicated in Chen Ch91 that, if the system is endowed with a strictly convex entropy, then, for any $C^{2}$ entropy pair $(\eta, q)$, there exists $\mu_{\eta} \in \mathcal{M}\left(\mathbb{R}_{+} \times \mathbb{R}^{n}\right)$ such that

$$
\operatorname{div}_{(t, x)}(\eta(u(t, x)), q(u(t, x)))=\mu_{\eta} .
$$

We introduce a functional on any oriented surface $S$ :

$$
\mathcal{F}_{\eta}(S)=\int_{S}(\eta(u), q(u)) \cdot \boldsymbol{\nu} d \mathcal{H}^{n},
$$

where $(\eta(u), q(u)) \cdot \boldsymbol{\nu}$ is the normal trace in the sense of Theorem 21.1, since $(\eta(u), q(u)) \in \mathcal{D} \mathcal{M}_{\text {loc }}^{\infty}\left(\mathbb{R}_{+} \times\right.$ $\mathbb{R}^{n}$ ). It is easy to check that the functional $\mathcal{F}_{\eta}$ defined by (39.4) is a Cauchy flux in the sense of Definition 32.2 .

39.2. Definition (Cauchy Entropy Fluxes). A functional $\mathcal{F}_{\eta}$ defined by (39.4) is called a Cauchy entropy flux with respect to the entropy $\eta$. 
In particular, when $\eta$ is convex, then

$$
\mathcal{F}_{\eta}(S) \geq 0
$$

for any oriented surface $S$. Furthermore, we can reformulate the balance law of entropy from the recovery of an entropy production by capturing entropy dissipation.

On the other hand, it is clear that understanding more properties of divergence-measure fields can advance our understanding of the behavior of entropy solutions for hyperbolic conservation laws and other related nonlinear equations by selecting appropriate entropy pairs. As examples, we refer the reader to [CF99a, CF99b, CF03, CW02 for the stability of Riemann solutions, which may contain rarefaction waves, contact discontinuities, and/or vacuum states, in the class of entropy solutions of the Euler equations for gas dynamics; to CF99c, CW02 for the decay of periodic entropy solutions for hyperbolic conservation laws; to CR00, Va01 for the initial and boundary layer problems for hyperbolic conservation laws; to CF99a, CT05 for the initial-boundary value problems for hyperbolic conservation laws; and to BFK02, MPT02 for nonlinear degenerate parabolic-hyperbolic equations.

It is hoped that the theory of divergence-measure fields could be used to develop techniques in entropy methods, measure-theoretic analysis, partial differential equations, and related areas.

Acknowledgments. The authors would like to thank Luis Caffarelli, Constantine Dafermos, Willi Jäger, Fanghua Lin, Leon Simon, and David Swanson for stimulating and fruitful discussions. GuiQiang Chen's research was supported in part by the National Science Foundation under Grants DMS-0505473, DMS-0244473, and an Alexander von Humboldt Foundation Fellowship. Monica Torres's research was supported in part by the National Science Foundation under grant DMS0540869 .

\section{REFERENCES}

[AFP00] L. Ambrosio, N. Fusco, and D. Pallara. Functions of Bounded Variation and Free Discontinuity Problems. Oxford Mathematical Monographs. The Clarendon Press, Oxford University Press: New York, 2000.

[Anz83] G. Anzellotti. Pairings between measures and bounded functions and compensated compactness. Ann. Mat. Pura Appl. (4), 135:293-318 (1984), 1983.

[AS56a] N. Aronszajn and K. T. Smith. Functional spaces and functional completion. Ann. Inst. Fourier. Grenoble, 6:125-185, 1955-1956.

[BC84] C. Baiocchi and A. Capelo. Variational and Quasivariational Inequalities. John Wiley \& Sons Inc.: New York, 1984.

[Bes44] A. S. Besicovitch. On the definition of the area of a surface by means of inscribed polyhedra. J. London Math. Soc., 19:138-141, 1944.

[BB02] J. Bourgain and H. Brezis, On the equation $\operatorname{div} Y=f$ and applications to control of phases, $\underline{\mathrm{J} \text {. Amer. }}$ Math. Soc., 16(1): 393-426, 2002.

[BF91] F. Brezzi and M. Fortin. Mixed and Hybrid Finite Element Methods. Springer-Verlag: New York, 1991.

[BM69] Yu. D. Burago and V. G. Maz'ya. Potential Theory and Function Theory for Irregular Regions. Translated from Russian. Seminars in Mathematics, V. A. Steklov Mathematical Institute, Leningrad, Vol. 3. Consultants Bureau, New York, 1969.

[BFK02] R. Bürger, H. Frid, and K. H. Karlsen. On a free boundary problem for a strongly degenerate quasilinear parabolic equation with an application to a model of pressure filtration. SIAM J. Math. Anal., 34(3):611-635 (electronic), 2002.

[Ca1823] A. L. Cauchy. Recherches sur l'équilibre et le mouvement intérieur des corps solides ou fluides, élastiques or non élastiques. Bull. Soc. Philomathique, 10(2):9-13, 1823.

[Ca1827] A. L. Cauchy. Da la pression ou tension dans un corps solide. Exercises de Matehématiques, 2(2):42-56, 1827.

[Ch91] G.-Q. Chen. Hyperbolic systems of conservation laws with a symmetry. Commun. Partial Diff. Eqs., 16:1461-1487, 1991.

[CF99a] G.-Q. Chen and H. Frid. Divergence-measure fields and hyperbolic conservation laws. Arch. Ration. Mech. Anal., 147(2):89-118, 1999.

[CF99b] G.-Q. Chen and H. Frid. Large-time behavior of entropy solutions of conservation laws. J. Diff. Eqs., 152(2):308-357, 1999.

[CF99c] G.-Q. Chen and H. Frid. Decay of entropy solutions of nonlinear conservation laws. Arch. Rational Mech. Anal., 146(2):95-127, 1999. 
[CF03] G.-Q. Chen and H. Frid. Extended divergence-measure fields and the Euler equations for gas dynamics. Commun. Math. Phys., 236(2):251-280, 2003.

[CR00] G.-Q. Chen and M. Rascle. Initial layers and uniqueness of weak entropy solutions to hyperbolic conservation laws. Arch. Rational Mech. Anal., 153(3):205-220, 2000.

[CT05] G.-Q. Chen and M. Torres. Divergence-measure fields, sets of finite perimeter, and conservation laws. Arch. Rational Mech. Anal., 175(2):245-267, 2005.

[CTZb] G.-Q. Chen, M. Torres, and W. P. Ziemer. Measure-theoretic analysis and nonlinear conservation laws. Pure Appl. Math. Quarterly, 2007 (to appear).

[CW02] G.-Q. Chen and D.-H. Wang. The Cauchy problem for the Euler equations for compressible fluids. In Handbook of Mathematical Fluid Dynamics, Vol. I, pages 421-543. North-Holland: Amsterdam, 2002.

[Daf98] C. M. Dafermos. Balance laws in continuum physics. In: Advanced Topics in Theoretical Fluid Mechanics (Paseky nad Jizerou, 1997), Pitman Res. Notes Math. Ser., 392, pages 89-117. Longman: Harlow, 1998.

[Daf05] C. M. Dafermos. Hyperbolic Conservation Laws in Continuum Physics, 2nd Ed., Springer-Verlag: Berlin, 2005.

[DG61a] E. De Giorgi. Complementi alla teoria della misura $(n-1)$-dimensionale in uno spazio $n$-dimensionale. Seminario di Matematica della Scuola Normale Superiore di Pisa, 1960-61. Editrice Tecnico Scientifica, Pisa, 1961.

[DG61b] E. De Giorgi. Frontiere orientate di misura minima. Seminario di Matematica della Scuola Normale Superiore di Pisa, 1960-61. Editrice Tecnico Scientifica, Pisa, 1961.

[DMM99] M. Degiovanni, A. Marzocci, and A. Musesti. Cauchy fluxes associated with tensor fields having divergence measure. Arch. Rational Mech. Anal., 147(3):197-223, 1999.

[DPP04a] T. De Pauw and W. F. Pfeffer. The Gauss-Green theorem and removable sets for PDEs in divergence form. Adv. Math., 183(1):155-182, 2004.

[EG92] L. C. Evans and R. F. Gariepy. Measure Theory and Fine Properties of Functions. Studies in Advanced Mathematics. CRC Press: Boca Raton, FL, 1992.

[Fed45] H. Federer. The Gauss-Green theorem. Trans. Amer. Math. Soc., 58:44-76, 1945.

[Fed58] H. Federer. A note on the Gauss-Green theorem. Proc. Amer. Math. Soc., 9:447-451, 1958.

[Fed59] H. Federer. Curvature measures. Trans. Amer. Math. Soc., 93:418-491, 1959.

[Fed66] H. Federer. Two theorems in geometric measure theory. Bull. Amer. Math. Soc., 72:719, 1966.

[Fed68b] H. Federer. Some properties of distributions whose partial derivatives are representable by integration. Bull. Amer. Math. Soc., 74:183-186, 1968.

[Fed69] H. Federer. Geometric Measure Theory. Die Grundlehren der Mathematischen Wissenschaften, Band 153. Springer-Verlag New York Inc.: New York, 1969.

[FF60] H. Federer and W. H. Fleming. Normal and integral currents. Ann. Math. (2), 72:458-520, 1960.

[FZ73a] H. Federer and W. P. Ziemer. The Lebesgue set of a function whose distribution derivatives are $p$-th power summable. Indiana Univ. Math. J., 22:139-158, 1972/73.

[Fle60] W. H. Fleming. Functions whose partial derivatives are measures. Illinois J. Math., 4:452-478, 1960.

[FL71] K. O. Friedrichs and P. D. Lax. Systems of conservation equations with a convex extension. Proc. Nat. Acad. Sci. U.S.A., 68:1686-1688, 1971.

[Fug55] B. Fuglede. On a theorem of F. Riesz. Math. Scand., 3:283-302 (1956), 1955.

[Fug60] B. Fuglede. Extremal Length and Closed Extensions of Partial Differential Operators. Jul. Gjellerups Boghandel, Copenhagen, 1960.

[Giu77] E. Giusti. Minimal Surfaces and Functions of Bounded Variation. With notes by G. H. Williams, Notes on Pure Mathematics, 10, Department of Pure Mathematics, Australian National University, Canberra, 1977.

[GNN61] C. Goffman, C. J. Neugebauer, and T. Nishiura. Density topology and approximate continuity. Duke Math. J., 28:497-505, 1961.

[GW61] C. Goffman and D. Waterman. Approximately continuous transformations. Proc. Amer. Math. Soc., 12:116-121, 1961.

[GM76] M. E. Gurtin and L. C. Martins. Cauchy's theorem in classical physics. Arch. Rational Mech. Anal., 60(4):305-324, 1975/76.

[GW67] M. E. Gurtin and W. O. Williams. An axiomatic foundation for continuum thermodynamics. Arch. Rational Mech. Anal., 26:83-117, 1967.

[GW71] M. E. Gurtin and W. O. Williams. On the first law of thermodynamics. Arch. Rational Mech. Anal., 42:77-92, 1971.

[GWZ86] M. E. Gurtin, W. O. Williams, and W. P. Ziemer. Geometric measure theory and the axioms of continuum thermodynamics. Arch. Rational Mech. Anal., 92(1):1-22, 1986.

[Gus60] W. Gustin. Boxing inequalities. J. Math. Mech., 9:229-239, 1960.

[He07] J. Heinonen. Nonsmooth calculus. Bull. Amer. Math. Soc., 1:632-232, 2007.

[JN90] W. B. Jurkat and D. J. F. Nonnenmacher. The general form of Green's theorem. Proc. Amer. Math. Soc., 109(4):1003-1009, 1990. 
[JN94a] W. B. Jurkat and D. J. F. Nonnenmacher. An axiomatic theory of non-absolutely convergent integrals in $\mathbf{R}^{n}$. Fund. Math., 145(3):221-242, 1994.

[JN94b] W. B. Jurkat and D. J. F. Nonnenmacher. A generalized $n$-dimensional Riemann integral and the divergence theorem with singularities. Acta Sci. Math. (Szeged), 59(1-2):241-256, 1994.

[JN94c] W. B. Jurkat and D. J. F. Nonnenmacher. A theory of non-absolutely convergent integrals in $\mathbf{R}^{n}$ with singularities on a regular boundary. Fund. Math., 146(1):69-84, 1994.

[JN95] W. B. Jurkat and D. J. F. Nonnenmacher. A Hake-type property for the $\nu_{1}$-integral and its relation to other integration processes. Czechoslovak Math. J., 45(120)(3):465-472, 1995.

[JNm95] W. B. Jurkat and D. J. F. Nonnenmacher. The fundamental theorem for the $\nu_{1}$-integral on more general sets and a corresponding divergence theorem with singularities. Czech. Math. J., 45(120)(1):69-77, 1995.

[Lew88a] J. L. Lewis. Uniformly fat sets. Trans. Amer. Math. Soc., 308(1):177-196, 1988.

[LY02] F. H. Lin and X. P. Yang. Geometric Measure Theory-An Introduction. Science Press: Beijing; International Press: Boston, MA, 2002.

[LRX07] A. Lim, B. Rodrigues, and Zhou Xu. A $m$-parallel crane scheduling problem with a non-crossing constraint. Naval Res. Logist., 54(2):115-127, 2007.

[MPT02] C. Mascia, A. Porretta, and A. Terracina. Nonhomogeneous Dirichlet problems for degenerate parabolichyperbolic equations. Arch. Rational Mech. Anal., 163(2):87-124, 2002.

[Mor47] A. P. Morse. Perfect blankets. Trans. Amer. Math. Soc., 61:418-442, 1947.

[No159] W. Noll. The foundations of classical mechanics in the light of recent advances in continuum mechanics. In: The Axiomatic Method. With Special Reference to Geometry and Physics. Proceedings of an International Symposium held at the Univ. of Calif., Berkeley, Dec. 26, 1957-Jan. 4, 1958 (edited by L. Henkin, P. Suppes and A. Tarski), Studies in Logic and the Foundations of Mathematics, pages 266-281, NorthHolland Publishing Co.: Amsterdam, 1959.

[Nol72] W. Noll. A new mathematical theory of simple materials. Arch. Rational Mech. Anal., 48:1-50, 1972.

[Nol73] W. Noll. Lectures on the foundations of continuum mechanics and thermodynamics. Arch. Rational Mech. Anal., 52:62-92, 1973.

[Nol86] W. Noll. Continuum mechanics and geometric integration theory. In: Categories in Continuum Physics (Buffalo, N.Y., 1982), Lecture Notes in Math., 1174, pages 17-29. Springer: Berlin, 1986.

[Nol87] W. Noll. Finite-Dimensional Spaces. Vol. I, Mechanics: Analysis, 10. Martinus Nijhoff Publishers: Dordrecht, 1987.

[Non95] D. J. F. Nonnenmacher. Sets of finite perimeter and the Gauss-Green theorem with singularities. J. London Math. Soc. (2), 52(2):335-344, 1995.

[Pfe] W. F. Pfeffer. Distributions for which $\operatorname{div} v=f$ has a continuous solution. To Appear.

[Pfe01] W. F. Pfeffer. The Stokes theorem for the generalized Riemann integral. Real Anal. Exchange, 26(2):623636, 2000/01.

[Pfe90] W. F. Pfeffer. Divergence theorem for vector fields with singularities. In: New Integrals (Coleraine, 1988), Lecture Notes in Math., 1419, pp. 150-166, Springer: Berlin, 1990.

[Pfe05a] W. F. Pfeffer. The Gauss-Green theorem in the context of Lebesgue integration. Bull. London Math. Soc., 37(1):81-94, 2005.

[Sch07] F. Schuricht. A new mathematical foundation for contact interactions in continnum physics. Arch. Rational Mech. Anal., 184: 495-551, 2007.

[Sim83] L. Simon. Lectures on Geometric Measure Theory, Proceedings of the Centre for Mathematical Analysis, 3, Australian National University, Canberra, 1983.

[Ste93] E. M. Stein. Harmonic Analysis: Real-Variable Methods, Orthogonality, and Oscillatory Integrals. Princeton Mathematical Series, 43. Princeton University Press: Princeton, NJ, 1993.

[Va01] A. Vasseur. Strong traces for solutions of multidimensional scalar conservation laws. Arch. Rational Mech. Anal., 160(3):181-193, 2001.

[Vol67a] A. I. Vol'pert. Spaces BV and quasilinear equations. Mat. Sb. (N.S.), 73 (115):255-302, 1967.

[VH69a] A. I. Vol'pert and S. I. Hudjaev. The Cauchy problem for second order quasilinear degenerate parabolic equations. Mat. Sb. (N.S.), 78 (120):374-396, 1969.

[Whi57] H. Whitney. Geometric Integration Theory. Princeton University Press: Princeton, N. J., 1957.

[Zie67] W. P. Ziemer. Extremal length and conformal capacity. Trans. Amer. Math. Soc., 126:460-473, 1967.

[Zie69] W. P. Ziemer. Extremal length and p-capacity. Michigan Math. J., 16:43-51, 1969.

[Zie70] W. P. Ziemer. Extremal length as a capacity. Michigan Math. J., 17:117-128, 1970.

[Zie83] W. P. Ziemer. Cauchy flux and sets of finite perimeter. Arch. Rational Mech. Anal., 84(3):189-201, 1983.

[Zie89] W. P. Ziemer. Weakly Differentiable Functions, Graduate Texts in Mathematics, 120, Springer-Verlag: New York, 1989.

(G.-Q. Chen) Department of Mathematics, Northwestern University, 2033 Sheridan Road, Evanston, IL 60208-2730, USA. HTTP://WWW.MATH.NORTHWESTERN.EDU/ GQCHEN

E-mail address: gqchen@math.northwestern.edu 
(M. Torres) Department of Mathematics, Purdue University, 150 N. University Street, West Layayette, IN 47907-2067, USA. HTTP://WWW.MATH.PURDUE.EDU/ ${ }^{\text {TORRES }}$

E-mail address: torres@math.purdue.edu

(W. Ziemer) Department of Mathematics, Indiana University, Rawles Hall, Bloomington, IN 47405, USA. HTTP://WWW.INDIAND.EDU/ ${ }^{2}$ ZIEMER

E-mail address: ziemer@indiana.edu 